\title{
Special $L$-values of geometric motives
}

\author{
Jakob Scholbach *
}

October 28, 2018

\begin{abstract}
This paper proposes a conceptual unification of Beilinson's conjecture about special $L$-values for motives over $\mathbb{Q}$, the Tate conjecture over $\mathbb{F}_{p}$ and Soulé's conjecture about pole orders of $\zeta$-functions of schemes over $\mathbb{Z}$. We conjecture the following: the order of $L(M, s)$ at $s=0$ is given by the negative Euler characteristic of motivic cohomology of $M^{\vee}(-1)$. Up to a nonzero rational factor, the $L$-value at $s=0$ is given by the determinant of the pairing of Arakelov motivic cohomology of $M$ with the motivic homology of $M$ :

$$
L^{*}(M, 0) \equiv \prod_{i \in \mathbb{Z}} \operatorname{det}\left(\mathrm{H}_{i-2}(M,-1) \otimes \widehat{\mathrm{H}}^{i}(M) \rightarrow \mathbb{R}\right)^{(-1)^{i+1}}\left(\bmod \mathbb{Q}^{\times}\right) .
$$

Under standard assumptions concerning mixed motives over $\mathbb{Q}, \mathbb{F}_{p}$, and $\mathbb{Z}$, this conjecture is equivalent to the conjunction of the above-mentioned conjectures of Beilinson, Tate, and Soulé. We use this to unconditionally prove the Beilinson conjecture for all Tate motives and, up to an $n$-th root of a rational number, for all Artin-Tate motives.
\end{abstract}

In this paper, we study special values of $L$-functions of geometric motives over $\mathbb{Z}$. This contains both $L$-functions over $\mathbb{Q}$ and Hasse-Weil $\zeta$-functions of schemes $X$ of finite type over $\mathbb{Z}$ (Propositions 3.5, 3.7]):

$$
\begin{gathered}
L_{\mathbb{Q}}\left(M_{\eta}, s\right)^{-1}=L_{\mathbb{Z}}\left(\eta_{! *} M_{\eta}[1], s\right), \\
\zeta(X, s)=L\left(\mathrm{M}_{\mathrm{c}}(X), s\right) .
\end{gathered}
$$

Here $M_{\eta}$ is a mixed motive over $\mathbb{Q}, \eta_{! *}$ is a generic intermediate extension functor similar to the one familiar in perverse sheaf theory, and $\mathrm{M}_{\mathrm{c}}(X)$ denotes the motive with compact support.

Our conjecture on special $L$-values is as follows:

Conjecture 0.1. (see Conjectures 4.1 and 5.2) Let $M$ be any geometric motive over $\mathbb{Z}$. We conjecture that pole orders are given by the negative Euler characteristic of motivic cohomology of $M^{\vee}(-1)$ :

$$
\operatorname{ord}_{s=0} L(M, s)=-\chi\left(M^{\vee}(-1)\right) .
$$

We conjecture that the Arakelov intersection pairing, which is the natural pairing of $\mathbb{R}$-vector spaces

$$
\pi_{M}: \underbrace{\operatorname{Hom}(\mathbf{1}(-1)[-2], M)}_{=: \mathrm{H}_{-2}(M,-1)} \times \underbrace{\operatorname{Hom}(M, \widehat{\mathbf{1}})}_{=: \widehat{\mathrm{H}}^{0}(M)} \stackrel{\circ}{\longrightarrow} \operatorname{Hom}(\mathbf{1}, \widehat{\mathbf{1}}(1)[2])=\mathbb{R}
$$

involving the motivic homology and the Arakelov motivic cohomology of $M$ is a perfect pairing of finite-dimensional $\mathbb{R}$-vector spaces. This conjectural perfectness

*Universität Münster, Germany 
is very interesting in its own right. For example, special cases of it are equivalent to the Beilinson-Soulé vanishing conjecture (Theorem 4.5) and the Beilinson-Parshin conjecture (Theorem 4.3). It also allows to equivalently reformulate (0.2) using the Euler characteristic of Arakelov motivic cohomology:

$$
\operatorname{ord}_{s=0} L(M, s)=-\widehat{\chi}(M) .
$$

Most importantly, though, it allows to express the following conjecture for the special $L$-value $L^{*}(M, 0)$ up to a nonzero rational factor, using the determinants of the pairings $\pi_{M[i]}$ :

$$
L^{*}(M, 0) \equiv \Pi_{M}^{-1}\left(\bmod \mathbb{Q}^{\times}\right)
$$

where

$$
\Pi_{M}:=\prod_{i \in \mathbb{Z}} \operatorname{det}\left(\pi_{M[i]}\right)^{(-1)^{i}}\left(\in \mathbb{R}^{\times} / \mathbb{Q}^{\times}\right) .
$$

The Arakelov motivic cohomology referred to above is a new cohomology established in HS11, Sch12a, (or see Section 2.2). It can be thought of as a cohomology with compact support, where "compact" refers to the compactification of Spec $\mathbb{Z}$. More precisely, it is characterized by a long exact sequence

$$
\ldots \rightarrow \widehat{\mathrm{H}}^{n}(M) \rightarrow \mathrm{H}^{n}(M) \stackrel{\text { ch }}{\rightarrow} \mathrm{H}_{\mathrm{D}}^{n}(M) \rightarrow \widehat{\mathrm{H}}^{n+1}(M) \rightarrow \ldots
$$

involving the Chern class map ch (also known as the Beilinson regulator) between motivic cohomology and Deligne cohomology.

This conjecture is related to existing conjectures on $L$-functions as follows:

Theorem 0.2. (see Theorems 5.8, 5.9 for the precise statements) Assuming the existence of the category of mixed motives (see Axiom 1.2), Conjecture 0.1 is essentially equivalent to the conjunction of the conjectures 5.14, 5.11, 5.19 of Beilinson, Soulé and Tate on special L-values of motives over $\mathbb{Q}$ and $\zeta$-functions à la HasseWeil of schemes over $\mathbb{Z}$ and over $\mathbb{F}_{p}$, respectively.

Recall that the subcategory $\operatorname{DATM}(\mathbb{Z})$ of Artin-Tate motives is the triangulated subcategory generated by direct summands of motives of number rings $\mathcal{O}_{F}$ and finite fields $\mathbb{F}_{q}$. Only allowing $\mathbb{Q}$ and $\mathbb{F}_{p}$ instead of arbitrary $\mathcal{O}_{F}$ and $\mathbb{F}_{q}$, we get the triangulated category $\mathbf{D T M}(\mathbb{Z})$ of Tate motives. Note that these motives have rational coefficients. These categories do enjoy a motivic t-structure whose hearts are denoted $\operatorname{MATM}(\mathbb{Z})$ and $\operatorname{MTM}(\mathbb{Z})$, respectively Sch11. We get the following unconditional result:

Corollary 0.3. The perfectness of the Arakelov intersection pairing, as well as the pole order formula (0.2) holds for any Artin-Tate motive over $\mathbb{Z}$. The formula for the special L-value holds for all motives in the triangulated category generated by motives $\mathrm{M}\left(\mathcal{O}_{F}\right)$ and $\mathrm{M}\left(\mathbb{F}_{q}\right)$, in particular for any Tate motive, i.e., any motive in $\operatorname{DTM}(\mathbb{Z})$. More generally, for any $M \in \operatorname{DATM}(\mathbb{Z})$,

$$
L^{*}(M, 0) \cdot \Pi_{M}
$$

is a torsion element of $\mathbb{R}^{\times} / \mathbb{Q}^{\times}$.

In particular, Beilinson's conjecture holds for any smooth projective variety $X_{\eta} / \mathbb{Q}$ such that $\mathrm{h}^{j}\left(X_{\eta}\right)$ is a mixed Tate motive $(j \in \mathbb{Z})$. Examples of such varieties include linear varieties [Jan90, Section 14], [Tot14], such as toric varieties and Grassmannians. Similarly, Beilinson's conjecture holds up to the $m$-th root of a nonzero rational number if $\mathrm{h}^{j}\left(X_{\eta}\right)$ is a mixed Artin-Tate motive. 
Proof: We first show that for any $M \in \operatorname{DATM}(\mathbb{Z})$, there is some $m>0$ such that $m M:=M^{\oplus m}$ lies in the triangulated subcategory $L \subset \mathbf{D A T M}(\mathbb{Z})$ generated by motives of the form $\mathrm{M}\left(\mathcal{O}_{F}\right)(n)[1]$ and direct factors of $\mathrm{M}\left(\mathbb{F}_{q}\right)$, for any $q=p^{r}, n \in \mathbb{Z}$ and any number ring $\mathcal{O}_{F}$. This statement is unrelated to the Arakelov intersection pairing and $L$-functions. It is enough to show this for $M$ being a direct summand of $\mathrm{M}\left(\mathcal{O}_{F}\right)(n)[1]$. By definition of $\eta_{! *}$, see [Sch12b, Section 5.4], $M^{\prime}:=\eta_{! *} \eta^{*} M$ lies in the triangulated category generated by $M$ and motives of the form $i_{*} N$, where $N \in \operatorname{DATM}\left(\mathbb{F}_{p}\right)$ and $i: \operatorname{Spec} \mathbb{F}_{p} \rightarrow \operatorname{Spec} \mathbb{Z}$. As $i_{*} N \in L$ for all $N \in \operatorname{DATM}\left(\mathbb{F}_{p}\right)$, it is enough to show $m M^{\prime} \in L$. Note that $M_{\eta}:=\eta^{*} M[-1]$ is a direct summand of $\mathrm{M}(F)(n)$. After twisting by $-n$, these two motives are Artin motives over $\mathbb{Q}$ (with rational coefficients). This category is equivalent to continuous rational $\operatorname{Gal}(\mathbb{Q})$ representations. For some finite quotient $G=\operatorname{Gal}(E / \mathbb{Q})$ of $\operatorname{Gal}(\mathbb{Q}), \mathrm{M}(F)$ and $M_{\eta}$ factor over $G$. By Artin induction [Ser78, II.13.1, Théorème 30], there is an equality in $K_{0}(\mathbb{Q}[G])$, the $K_{0}$-group of the group ring of $G$ (with rational coefficients) $m\left[M_{\eta}(-n)\right]=\sum_{i} l_{i}\left[\operatorname{ind}_{H}^{G} \mathbb{Q}\right]$, where $m, l_{i} \in \mathbb{Z}, m>0$, and $H$ runs over the cyclic subgroups of $G$. The functor $\eta_{! *}[1]$ does not in general send a short exact sequence

$$
E_{\eta}: 0 \rightarrow M_{\eta, 1} \rightarrow M_{\eta, 2} \rightarrow M_{\eta, 3} \rightarrow 0
$$

in $\operatorname{MATM}(\mathbb{Q})$ to a distinguished triangle in $\operatorname{DATM}(\mathbb{Q})$. However, for a sufficiently small open $j: U \subset$ Spec $\mathbb{Z}$, there is a similar short exact sequence $E_{U}$ in $\operatorname{MATM}(U)$ such that $\eta^{*} E_{U}[-1]=E_{\eta}$ and such that $\eta_{! *} M_{\eta, n}[1]=j_{! *} M_{U, n}$ for all $n$. As $j_{\text {! }}$ is triangulated, $j_{!}\left(E_{U}\right)$ is a distinguished triangle in $\operatorname{DATM}(\mathbb{Z})$. Moreover, $j_{! *} M_{U, n}$ lies in a distinguished triangle whose other vertices are $j_{!} M_{U, n}$ and $i_{*} N$, where $i: Z \rightarrow \operatorname{Spec} \mathbb{Z}$ is the complement of $j$ and $N \in \operatorname{DATM}(Z)$. Therefore, if $\eta_{! *} M_{\eta, j}[1] \in L$ for two out of the three $M_{\eta, j}$ 's, it is true for the third. Noting that $\operatorname{ind}_{H}^{G} \mathbb{Q}$ corresponds to the motive $\mathrm{M}\left(E^{H}\right)$ of the subfield $E^{H} \subset E$ fixed by $H$ and $\eta_{! *} \mathrm{M}\left(E^{H}\right)[1]=\mathrm{M}\left(\mathcal{O}_{E^{H}}\right)[1] \in L$, we obtain $m \eta_{! *} M_{\eta}[1] \in L$.

For any number field $F$ and number ring $\mathcal{O}_{F}$, the conjectured pole order formula, the special value and the perfectness of the Arakelov intersection pairings for $\mathrm{M}\left(\mathcal{O}_{F}\right)(n)[1]$ are (unconditionally, by Proposition 5.16, Remark 5.17 and Theorem 5.18) equivalent to Beilinson's conjecture for $\mathrm{M}(F)(n) \in \operatorname{MATM}(\mathbb{Q})$ which does hold by Borel's work Bor77. The three conjectures also hold for direct factors of $\mathrm{M}\left(\mathbb{F}_{q}\right)$ by Quillen's computation of $K$-theory of finite fields Qui72. By Theorem 5.5. the three conjectures therefore hold for any motive in $L \subset \operatorname{DATM}(\mathbb{Z})$.

Now, let $M \in \mathbf{D A T M}(\mathbb{Z})$ be any Artin-Tate motive. There is an $m>0$ such that $m M \in L$. Since the Arakelov intersection pairings are induced by the composition of morphisms in $\mathbf{D M}_{\mathrm{5}}(\mathbb{Z})$, the map $r_{m M}: \mathrm{H}_{-2}(m M,-1) \rightarrow \widehat{\mathrm{H}}^{0}(m M)^{\vee}$ induced by $\pi_{m M}$ is clearly the $m$-fold direct sum of the map $r_{M}$ induced by $\pi_{M}$. Hence the perfectness of $\pi_{m M}$, i.e., $r_{m M}$ being an isomorphism, implies the perfectness of $\pi_{M}$. Moreover, we have $\left(L^{*}(M, 0) \Pi_{M}\right)^{m}=L^{*}(m M, 0) \Pi_{m M}=1 \in \mathbb{R}^{\times} / \mathbb{Q}^{\times}$, i.e., $L^{*}(M, 0) \Pi_{M}$ is torsion in $\mathbb{R}^{\times} / \mathbb{Q}^{\times}$. Similarly, $m\left(\operatorname{ord}_{s=0} L(M, s)+\chi\left(M^{\vee}(-1)\right)\right)=$ $\operatorname{ord}_{s=0} L(m M, s)+\chi\left(m M^{\vee}(-1)\right)=0 \in \mathbb{Z}$, so that $\operatorname{ord}_{s=0} L(M, s)+\chi\left(M^{\vee}(-1)\right)=0$, i.e., the pole order formula holds.

The last statement follows immediately.

Conjecture 0.1 is compatible with the functional equation of $L$-functions. It is also stable under distinguished triangles (Theorem 5.5). While the latter is a formal consequence of the setup, it is a key difference between our conjecture and Beilinson's conjecture for mixed motives over $\mathbb{Q}$. It allows to break up a motive into smaller pieces by means of distinguished triangles. This technique is unapplicable when working with Beilinson's original conjecture for motives over $\mathbb{Q}$. Moreover, Conjecture 0.1 gives more freedom because it allows to work in the larger category of all geometric motives, as opposed to just smooth and projective varieties. It should be noted, though, that the proof of the equivalence of Beilinson's $L$-value 
formula and Conjecture 5.2 is formal, so that proving Beilinson's conjecture for any example not covered by techniques such as the ones in Corollary 0.3 will require new ideas.

The idea of reinterpreting the data in Beilinson's conjecture in terms of motives over $\mathbb{Z}$ is due to Huber. More precisely, a mixed motive $M_{\eta}$ over $\mathbb{Q}$ corresponds to the mixed motive $\eta_{! *} M_{\eta}[1]$ over $\mathbb{Z}$. This is reified for $L$-functions by (0.1) and on the motivic side by an appropriate interpretation of $f$-cohomology [Sch12b. The non-multiplicativity of $L$-functions (cf. Remark 3.2) is explained by the failure of $\eta_{\text {!* }}$ to be exact. $L$-functions of motives over $\mathbb{Z}$ are multiplicative, though.

This non-multiplicativity, which is a heavy technical burden, has been addressed by Scholl by introducing a category $\operatorname{MM}(\mathbb{Q} / \mathbb{Z})$ of mixed motives over $\mathbb{Z}$ Sch91] (different from the one used here) by imposing non-ramification conditions. The (conjectural) value of the groups $\operatorname{Ext}_{\mathbf{M M}(\mathbb{Q} / \mathbb{Z})}^{a}\left(\mathbf{1}, \mathrm{h}^{b-1}\left(X_{\eta}, m\right)\right)$ is closely related to the computation of $\mathrm{H}^{*}\left(\eta ! * \mathrm{~h}^{-b+1}\left(X_{\eta},-m\right)[1]\right)$ (Theorem 1.3). As for the special $L$-values, a conjecture of Scholl $\left[\right.$ Sch91, Conj. C] says that some $M_{\eta} \in \mathbf{M M}(\mathbb{Q} / \mathbb{Z})$ is critical (i.e., its period map is an isomorphism, equivalently all weak Hodge cohomology groups $\mathrm{H}_{\mathrm{w}}^{*}\left(M_{\eta}\right)$ vanish) if

$$
\operatorname{Ext}_{\mathbf{M M}(\mathbb{Q} / \mathbb{Z})}^{a}\left(M_{\eta}, \mathbf{1}(1)\right)=\operatorname{Ext}_{\mathbf{M M}(\mathbb{Q} / \mathbb{Z})}^{a}\left(\mathbf{1}, M_{\eta}\right)=0 \text { for } a=0,1 .
$$

Moreover, a reduction technique transforming any motive $M_{\eta}$ into one satisfying these vanishings is given, so that Deligne's conjecture [Del79, Conj. 2.8.] concerning the $L$-value of critical motives can be applied. In similar spirit, the nonmultiplicativity of $L$-functions of motives over $\mathbb{Q}$ has been addressed by Fontaine and Perrin-Riou by introducing the notion of $f$-exact sequences, which are ones where one does save multiplicativity [FPR94, III.3.1.4]. However, such exact sequences seem to be hard to characterize. The formulation of Conjecture 0.1 resembles their approach; for example the pole order in op. cit. is expressed as an Euler characteristic of $f$-cohomology. Using a "cohomology with compact support" to predict special $L$-values was already suggested by Beilinson [Bel87, 5.10.F]. The category of motives over $\mathbb{Z}$ is both the appropriate home for this idea and allows for the strikingly compact and beautiful formulation of the $L$-values conjecture by overcoming the technical obstacles related to motives over $\mathbb{Q}$.

The idea to recast special $L$-values of motives as determinants of appropriate pairings was explored by Deninger and Nart [DN95, who show that the motivic height pairing of Sch94 can be represented by concatenating morphisms in the derived category of an appropriate category of motives.

Conjecture 0.1 is the first conjecture that predicts the special values of $\zeta(X)$ modulo $\mathbb{Q}^{\times}$at all places $(X / \mathbb{Z}$ regular projective; see Example 5.13). A reformulation of the Tamagawa number conjecture in terms of the Weil-étale cohomology due to Flach and Morin predicts the special value of $\zeta(X)$ at $s=0$ up to sign [FM12, Prop. 9.2]. It remains to explicitly compare the compatibility of the approach of op. cit. and Conjecture 0.1. I expect that similar techniques as the ones in this paper allow to refine Conjecture 0.1 to a conjectural $L$-values formula, up to sign, at all places. However, this remains to be done.

This paper has its origins in a part of my $\mathrm{PhD}$ thesis. It is a pleasure to thank Annette Huber for her advice during this time. I thank Andreas Holmstrom for the collaboration on Arakelov motivic cohomology [HS11. I also thank Denis-Charles Cisinski, Frédéric Déglise and Bruno Kahn for helpful conversations. Finally, I thank the referee for suggesting many improvements to this article, in particular concerning the formulation of Corollary 0.3 


\section{Preliminaries}

\subsection{Determinants and $\mathbb{Q}$-structures}

For any ring $R$, let $\underline{R}$ be the category of finitely generated $R$-modules. Let $K$ be a field. The determinant $\operatorname{det} V$ of $V \in \underline{K}$ is $\operatorname{det} V:=\Lambda^{\operatorname{dim} V} V$. Its $K$-dual is denoted $\operatorname{det}^{-1} V$. For $V_{*} \in \mathbf{D}^{\mathrm{b}}(\underline{K})$, the derived category, we set $\operatorname{det} V_{*}:=$ $\bigotimes_{i} \operatorname{det}^{(-1)^{i}} \mathrm{H}^{i}\left(V_{*}\right)$. We abbreviate $\operatorname{det} H^{*}:=\operatorname{det}^{(-1)^{i}} H^{i}$ for some $H^{i} \in \underline{K}, i \in \mathbb{Z}$.

Let $A, B \in \mathbb{Q}$ and let $f: A_{\mathbb{R}} \rightarrow B_{\mathbb{R}}$ be an $\mathbb{R}$-linear map. We do not assume that it respects the rational subspaces. The "usual" determinant of $f$, which is well-defined up to a nonzero rational factor agrees, modulo $\mathbb{Q}^{\times}$with the image of 1 under the map $\mathbb{Q} \cong \operatorname{det} A \otimes \operatorname{det}^{-1} B \rightarrow \operatorname{det} A_{\mathbb{R}} \otimes \operatorname{det}^{-1} B_{\mathbb{R}} \cong \mathbb{R}$. Here the right hand isomorphism is induced by $f$.

A complex with $\mathbb{Q}$-structure is a complex $V_{*}$ of $\mathbb{R}$-vector spaces that is quasiisomorphic to one in $\mathbf{D}^{\mathrm{b}}(\underline{\mathbb{R}})$ together with a non-zero map of $\mathbb{Q}$-vector spaces $d_{V_{*}}$ : $\mathbb{Q} \rightarrow \operatorname{det} V_{*}$. In concrete situations, we usually have a distinguished identification $\operatorname{det} V_{*} \cong \mathbb{R}$. In that case, we may also call det $V_{*}$ the real number that is the image of $1 \in \mathbb{Q}$ under $d_{V_{*}}$ and the given identification.

Maps of complexes with $\mathbb{Q}$-structures are usual maps of complexes; they are not required to be compatible with the map $d_{V_{*}}$. For a map $f: V_{*} \rightarrow W_{*}$ of complexes with $\mathbb{Q}$-structures the cone of $f$ is endowed with the following $\mathbb{Q}$-structure:

$$
\mathbb{Q}^{d_{W}} \stackrel{\otimes\left(d_{V}\right)^{-1}}{\longrightarrow} \operatorname{det} W_{*} \otimes \operatorname{det}^{-1} V_{*} \cong \operatorname{det} \operatorname{cone}(f)
$$

Define a category $\mathbf{D}^{\mathrm{b}}(\underline{\mathbb{R}})^{\mathbb{Q}-\text { det }}$ to consist of such complexes. Its morphisms are given by maps of complexes up to quasi-isomorphism (not necessarily respecting the $\mathbb{Q}$-structures). We say that a triangle $A \rightarrow B \rightarrow C$ of objects in $\mathbf{D}^{\mathrm{b}}(\underline{\mathbb{R}})^{\mathbb{Q} \text {-det }}$ is multiplicative if it is distinguished in $\mathbf{D}^{\mathrm{b}}(\underline{\mathbb{R}})$ after forgetting the $\mathbb{Q}$-structure and $\operatorname{det} B=\operatorname{det} A \operatorname{det} C$ in the sense that the following diagram (whose right hand isomorphism stems from the triangle) is commutative:

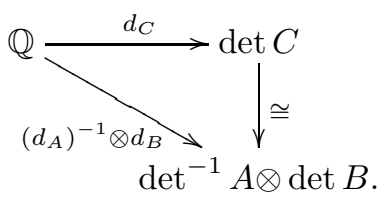

\subsection{Motives}

Our work takes place in the category $\mathbf{D M}_{\mathrm{5}}(S)$ of Beilinson motives over $S$, where $S$ is either a finite field, a number ring $\mathcal{O}_{F}$, or a number field $F$. Cisinski and Déglise defined this category to be an appropriate subcategory of Morel and Voevodsky's stable homotopy category $\mathbf{S H}(S)_{\mathbb{Q}}$ (with rational coefficients) CD09. The category $\mathbf{D M}_{\mathrm{Б}}(S)$ is tensor-triangulated, $\mathbb{Q}$-linear, and closed under arbitrary direct sums. Its tensor unit is denoted $\mathbf{1}_{S}$ or just $\mathbf{1}$. Given some scheme $f: X \rightarrow S$ (always tacitly supposed to be separated and of finite type), the motive of $X$ and the motive with compact support are defined as

$$
\mathrm{M}(X):=f_{!} f^{!} \mathbf{1}_{S}, \quad \mathrm{M}_{\mathrm{c}}(X):=f_{*} f^{!} \mathbf{1}_{S}
$$

Here $f_{!}: \mathbf{D M}_{\mathrm{Б}}(X) \rightarrow \mathbf{D M}_{\mathrm{Б}}(S)$ etc. are the functors defined in op. cit. This determines a covariant functor $\mathrm{M}: \mathbf{S c h} / S \rightarrow \mathbf{D M}_{\mathrm{B}}(S)$ and likewise, but just for proper maps, with $\mathrm{M}_{\mathrm{c}}$. The motive of the projective line decomposes as $\mathrm{M}\left(\mathbb{P}^{1}\right)=$ $\mathbf{1} \oplus \mathbf{1}(1)[2]$. In $\mathbf{D M}_{\mathrm{\zeta}}(S)$, tensoring with $\mathbf{1}(1)[2]$ is invertible and we write $M\{n\}:=$ 
$M \otimes(\mathbf{1}(1)[2])^{\otimes n}$ for any $n \in \mathbb{Z}$. For $M \in \mathbf{D M}_{\mathrm{b}}(S)$, we put

$$
\begin{aligned}
\mathrm{H}_{i}(M, p) & :=\operatorname{Hom}_{\mathbf{D M}_{\mathbf{B}}(S)}(\mathbf{1}(p)[i], M), \\
\mathrm{H}^{i}(M, p) & :=\operatorname{Hom}_{\mathbf{D M}_{\mathbf{B}}(S)}(M, \mathbf{1}(p)[i]) .
\end{aligned}
$$

For a regular base $S$ and a regular, projective or affine (but not necessarily flat) scheme $X$ over $S$ and $G:=\mathrm{M}(X)(-m)$, motivic cohomology of $X$ is given by

$\mathrm{H}^{i}(X, m):=\mathrm{H}^{i}(G)=\operatorname{Hom}_{S}\left(f_{!} f^{!} \mathbf{1}(-m), \mathbf{1}[i]\right)=\operatorname{Hom}_{X}(\mathbf{1}, \mathbf{1}(m)[i])=K_{2 m-i}(X)_{\mathbb{Q}}^{(m)}$,

using the purity isomorphism $f^{!} \mathbf{1}_{S}=f^{*} \mathbf{1}_{S}\{d\}=\mathbf{1}_{X}\{d\}$, where $d=\operatorname{dim} X-\operatorname{dim} S$. As a consequence of resolution of singularities, the full subcategory $\mathbf{D M}_{\mathrm{b}, c}(S) \subset$ $\mathbf{D M}_{\mathrm{b}}(S)$ of compact objects agrees with the thick subcategory generated by such motives $G$, for any base $S$ as above. We refer to objects of $\mathbf{D M}_{\mathrm{b}, c}(S)$ as geometric motives over $S$. For a perfect field $S$, there is a natural equivalence of categories CD09, Theorem 15.1.4]

$$
\mathbf{D M}_{\mathrm{b}, c}(S) \stackrel{\cong}{\longrightarrow} \mathbf{D M}_{\mathrm{gm}}(S)_{\mathbb{Q}}
$$

with Voevodsky's triangulated category of geometric motives (with rational coefficients) Voe00. It sends the motive $\mathrm{M}(X) \in \mathbf{D M}_{\mathrm{b}, c}(\mathbb{Q})$ of a smooth $S$-scheme in the sense of (1.1) to the motive $\mathrm{M}_{\mathrm{gm}}(X)$ of $X$ in Voevodsky's sense.

The category $\mathbf{D M}_{\mathrm{b}, c}(S)$ is equipped with a notion of weight: there are full (nontriangulated) subcategories $\mathbf{D} \mathbf{M}_{\mathrm{b}, c}^{\mathrm{wt} \leq n}(S), \mathbf{D M}_{\mathrm{b}, c}^{\mathrm{wt} \geq n}(S)$ such that $f_{!} \mathbf{1}(a)[2 a+n]$ lies in the subcategory

$$
\mathbf{D M}_{\mathrm{b}, c}^{\mathrm{wt}=n}(S):=\mathbf{D M}_{\mathrm{b}, c}^{\mathrm{wt} \leq n}(S) \cap \mathbf{D M}_{\mathrm{b}, c}^{\mathrm{wt} \geq n}(S)
$$

of objects of pure weight $n$, for all $a, n \in \mathbb{Z}$ and all proper maps $f: X \rightarrow S$ with regular domain $X$ [Bon10, Héb11]. For any map $f$ (of finite type), the functors $f_{!}$, $f^{*}$ preserve the subcategories $\mathbf{D M}_{\mathrm{b}, c}^{\mathrm{wt} \leq n}(-)$ and dually for $f^{!}, f^{*}$.

The dual of any geometric motive $M$ is defined as $M^{\vee}=\underline{\operatorname{Hom}}_{\mathbf{D M}_{\mathbf{B}}(S)}(M, \mathbf{1})$. Dualizing exchanges! and $*$ : for example, for any map $f,\left(f_{!} f^{!} \mathbf{1}\right)^{\vee}=f_{*} f^{*}\left(\mathbf{1}^{\vee}\right)$ which is canonically isomorphic $f_{*} f^{*} \mathbf{1}$. Therefore, the natural map

$$
M \rightarrow\left(M^{\vee}\right)^{\vee}
$$

is an isomorphism for any $M \in \mathbf{D M}_{\mathrm{b}, c}(\mathbb{Z})[\mathrm{CD09}$, 14.3.31]. This yields a canonical isomorphism $\mathrm{H}_{0}\left(M^{\vee}, 0\right)=\mathrm{H}^{0}(M, 0)$.

Definition 1.1. Let $S \subset \operatorname{Spec} \mathcal{O}_{F}$ be an open subscheme. A motive $M \in$ $\mathbf{D M}_{\mathrm{b}, c}(S)$ is called smooth if the natural map [Ayo07, Section 2.3.2] 1 ]

$$
i^{*} M\{-1\}=i^{*} M \otimes i^{!} \mathbf{1} \rightarrow i^{!}(M \otimes \mathbf{1})=i^{!} M
$$

is an isomorphism for all closed points $i:$ Spec $\mathbb{F}_{\mathfrak{p}} \rightarrow S$. A motive $M \in \mathbf{D M}_{\mathrm{b}, c}(S)$ is generically smooth if $j^{*} M$ is smooth for some open subscheme $j: U \subset S$.

Since $\mathrm{M}(X)(m)$ is smooth provided $X / S$ is smooth and proper, every motive $M \in \mathbf{D M}_{\mathrm{b}, c}(S)$ is generically smooth. We write $\eta: \operatorname{Spec} F \rightarrow \operatorname{Spec} \mathcal{O}_{F}$ for the generic point.

In order to interpret Beilinson's conjecture for mixed motives over $\mathbb{Q}$ in terms of motives over $\mathbb{Z}$ we need to assume the conjectural framework of mixed motives over $F$, Spec $\mathcal{O}_{F}$ and $\mathbb{F}_{q}$. The precise axioms we are staking on are listed in Sch12b,

\footnotetext{
${ }^{1}$ The use of this canonical map, as opposed to a mere noncanonical isomorphism, was suggested by Bruno Kahn.
} 
Section 4], so we only summarize them briefly and refer to loc. cit. for a more complete discussion. 23 Note that the corresponding statements for the triangulated category of Artin-Tate motives $\mathbf{D A T M}\left(\mathcal{O}_{F}\right) \subset \mathbf{D M}_{\mathrm{b}, c}\left(\mathcal{O}_{F}\right)$, which is the triangulated subcategory of $\mathbf{D M}_{\mathrm{b}}\left(\mathcal{O}_{F}\right)$ generated by $\mathrm{M}(V)(m)$ where $V \rightarrow \operatorname{Spec} \mathcal{O}_{F}$ is a quasi-finite, but not necessarily flat map and $m \in \mathbb{Z}$, have been shown in [Sch11.

Axiom 1.2. (i) Sch12b, Axioms 4.1, 4.2] $\mathbf{D M}_{\mathrm{b}, c}(S)$ is conjectured to enjoy a non-degenerate $t$-structure whose heart $\mathbf{M M}(S)$ is called the category of mixed motives. The cohomological dimension of $\mathbf{M M}(S)$ is conjectured to be 0 $\left(S=\mathbb{F}_{q}\right)$ and $1(S=F)$, respectively. The truncation with respect to the $t$-structure is denoted ${ }^{\mathrm{P}} \mathrm{H}^{*}$. We write $\mathrm{h}^{i}(X, n)$ for ${ }^{\mathrm{P}} \mathrm{H}^{i}(\mathrm{M}(X)(n))$. The $t$ structures are normalized by declaring $\mathbf{1} \in \mathbf{M M}(S)$ when $S=F, \mathbb{F}_{q}$ and $\mathbf{1}[1] \in$ $\operatorname{MM}\left(\mathcal{O}_{F}\right)$, respectively. For example, $\mathrm{h}^{-1}\left(\mathbb{P}_{\mathcal{O}_{F}}^{1}\right)=\mathbf{1}_{\mathcal{O}_{F}}(1)[1], \mathrm{h}^{-2}\left(\mathbb{P}_{F}^{1}\right)=$ $\mathbf{1}_{F}(1)$. More generally, $\eta^{*}[-1]$ is $t$-exact and $\eta^{*} \mathrm{~h}^{-b}(X,-m)=\mathrm{h}^{-b-1}\left(X_{\eta},-m\right)$ for any scheme $X / \mathcal{O}_{F}$ with generic fiber $X_{\eta}$.

(ii) Sch12b, Axiom 4.5.] The key requirement on the $t$-structure is that realization functors of the form $\mathbf{D M}_{\mathrm{b}, c}(S) \rightarrow \mathbf{D}^{\mathrm{b}}(\mathcal{C})$ are to be exact (see loc. cit. and around (1.7) for the $\ell$-adic realization over $\mathbb{Z}[1 / \ell]$ ). In the guise of a spectrum representing the cohomology theory, the exactness requirement is to be understood as in (2.14).

(iii) Sch12b, Axioms 4.4, 4.6, 4.11] Any mixed motive is conjectured to have a weight filtration which is compatible with the weight formalism mentioned around (1.3). The pure objects in $\mathbf{M M}(K)$ (for any field $K$ ) are conjectured to be identified with the category $\mathbf{M}_{\text {num }}$ of pure motives with respect to numerical equivalence. This implies that the pure objects in $\mathbf{M M}(K)$ form an abelian semi-simple category [Jan92, Th. 1]. Moreover, homological and numerical equivalence are conjectured to agree. The cohomology functors ${ }^{\mathrm{p}} \mathrm{H}^{*}$ belonging to the motivic $t$-structure are supposed to respect the weights, i.e., given some $M \in \mathbf{D M}_{\mathrm{b}, c}^{\mathrm{wt}=w},{ }^{\mathrm{p}} \mathrm{H}^{n}(M) \in \mathbf{M M}$ is pure of weight $w+n$. For example, for a smooth projective scheme $X / S, \mathrm{M}(X)(-m)=f_{!} f^{!} \mathbf{1}(-m) \in \mathbf{D M}_{\mathrm{b}, c}^{\mathrm{wt}=2 m}$, so that $\mathrm{h}^{-b}(X,-m)$ is pure of weight $2 m-b$. Morphisms of mixed motives are expected to respect weights strictly, thereby giving constraints on the existence of maps between motives.

In the remainder of this paper we assume that the axioms concerning mixed motives over open subschemes of Spec $\mathcal{O}_{F}, \mathbb{F}_{q}$ and $F$ hold.

Given a mixed motive over $\mathbb{Q}, M_{\eta} \in \mathbf{M M}(\mathbb{Q})$, pick any $M \in \mathbf{M M}(\mathbb{Z})$ satisfying $M_{\eta}=\eta^{*} M[-1]$ and some open subscheme $j: U \rightarrow$ Spec $\mathbb{Z}$ such that $j^{*} M$ is smooth. We call

$$
\eta_{! *}\left(M_{\eta}[1]\right):=j_{! *} j^{*} M:=\operatorname{im}\left(j ! j^{*} M \rightarrow j_{*} j^{*} M\right) \in \mathbf{M M}(\mathbb{Z})
$$

the generic intermediate extension of $M_{\eta}[1]$. This is explained and shown to be well-defined in [Sch12b, Section 5.4]. We apply this to $M_{\eta}=\mathrm{h}^{-b-1}\left(X_{\eta},-m\right)$ and $M=\mathrm{h}^{-b}(X,-m)$, where $X_{\eta} / \mathbb{Q}$ is smooth projective and $X / \mathbb{Z}$ is any projective (not necessarily regular) model of $X_{\eta}$ of constant dimension $d$. Throughout this

\footnotetext{
${ }^{2}$ Unlike this paper, op. cit. is written with a contravariant notation of motives. This induces a number of changes in notation: every $f_{!}, f^{!}$gets replaced by a $f_{*}$ and $f^{*}$, and vice versa. Moreover, a twist and shift $M(m)[n]$ corresponds to $M(-m)[-n]$ here. Both here and there, the normalization of the $t$-structure is such that $\mathbf{1}[1] \in \mathbf{M M}\left(\mathcal{O}_{F}\right)$, while $\mathbf{1} \in \mathbf{M M}(F)$.

3 The decomposition axiom for smooth projective varieties formulated in Sch12b, Axiom 4.13] is not needed: it is only used in Sch12b, Lemma 5.10] to show that a certain motive is generically smooth, but this is inconditionally true for any motive by the remark after Definition 1.1
} 
paper, we write

$$
E:=\eta_{! *} \eta^{*} \mathrm{~h}^{-b}(X,-m)=\eta_{! *}\left(\mathrm{~h}^{-b-1}\left(X_{\eta},-m\right)[1]\right) \in \mathbf{M M}(\mathbb{Z})
$$

This motive is pure of weight $w:=2 m-b$. Its motivic cohomology is given by the following theorem:

Theorem 1.3. With the above notation, we write $\mathrm{H}^{b}\left(X_{\eta}, m\right)_{\mathbb{Z}}:=\operatorname{im}\left(\mathrm{H}^{b}(X, m) \rightarrow\right.$ $\left.\mathrm{H}^{b}\left(X_{\eta}, m\right)\right)$. Moreover, let $\mathrm{CH}^{m}\left(X_{\eta}\right)_{\mathbb{Q}, \text { hom }}$ be the subgroup of the Chow group of cycles homologically equivalent to zero and $\mathrm{CH}^{m}\left(X_{\eta}\right)_{\mathbb{Q}} /$ hom the group of cycles modulo homological equivalence (tensored with $\mathbb{Q})$. Then

$$
\mathrm{H}^{a}(E)=\mathrm{H}^{a}\left(\eta_{! *} \mathrm{~h}^{-b-1}\left(X_{\eta},-m\right)[1]\right)= \begin{cases}\mathrm{CH}^{m}\left(X_{\eta}\right)_{\mathbb{Q}} / \mathrm{hom} & a=1, w=1 \\ 0 & a=1, w \neq 1 \\ 0 & a=2, w \leq 1 \\ \mathrm{CH}^{m}\left(X_{\eta}\right)_{\mathbb{Q}, \text { hom }} & a=2, w=2 \\ \mathrm{H}^{b+2}\left(X_{\eta}, m\right)_{\mathbb{Z}} & a=2, w \geq 3 \\ 0 & a=3, w \leq 2 \\ ? & a=3, w \geq 3 \\ 0 & a>3, a<1\end{cases}
$$

Proof: Everything except the cases $a=2, w \leq 1$ and $a=3, w \leq 2$ is shown in Sch12b, Lemma 5.2, Theorem 6.11]. For $a=2$ and $w \leq 1$, the map

$$
\mathrm{H}^{2}(E) \rightarrow \mathrm{H}^{2}\left(\eta^{*} E\right)=\mathrm{H}^{1}\left(\mathrm{~h}^{-b-1}\left(X_{\eta},-m\right)\right) \rightarrow \mathrm{H}^{b+2}\left(X_{\eta}, m\right)=\mathrm{CH}^{m}\left(X_{\eta}, w-2\right)=0
$$

is injective: for the first map this is [Sch12b, Lemma 6.9], the second one is because the cohomological dimension of $\mathbf{D M}_{\mathrm{5}, c}(\mathbb{Q})$ is one [Sch12b, Axiom 4.1.]. For $a=3$, $w \leq 2$, we use the exact localization sequence

$$
\ldots \rightarrow \oplus_{p} \mathrm{H}^{3}\left(i_{p *} i_{p}^{*} E\right) \rightarrow \mathrm{H}^{3}(E) \rightarrow \mathrm{H}^{3}\left(\eta^{*} E\right)=\mathrm{H}^{2}\left(\eta^{*}[-1] E\right)=0 .
$$

The right hand vanishing is again because the cohomological dimension of motives over $\mathbb{Q}$ being one. Also by cohomological dimension we have

$\mathrm{H}^{3}\left(i_{p *} i_{p}^{*} E\right)=\operatorname{Hom}\left(i_{p}^{*} E, i_{p}^{!} \mathbf{1}[3]\right)=\operatorname{Hom}\left(i_{p}^{*} E, \mathbf{1}(-1)[1]\right)=\operatorname{Hom}_{\mathbf{M M}\left(\mathbb{F}_{p}\right)}\left({ }^{\mathrm{p}} \mathrm{H}^{-1} i_{p}^{*} E(1), \mathbf{1}\right)$.

The functor $i^{*}$ preserves negative weights, i.e., $\operatorname{wt}\left({ }^{\mathrm{p}} \mathrm{H}^{-1}\left(i_{p}^{*} E(1)\right)\right) \leq \mathrm{wt}(E)-1-$ $2=w-3$. By strictness of the weight filtration the group therefore vanishes for $w \leq 2$.

In accordance with Conjecture 4.1 (see the case $w \leq 1$ in the proof of Proposition 5.16) I expect $\mathrm{H}^{3}(E)=0$ for arbitrary weight $w$. See the introduction for the relation of this to Scholl's notion of mixed motives over $\mathbb{Z}$. For Artin-Tate motives, the expected vanishing holds unconditionally for all weights:

Theorem 1.4. Let $M_{\eta}$ be an Artin-Tate motive over $F$, concentrated in cohomological degree -1 . Then $\mathrm{H}^{3}\left(\mathcal{O}_{F}, \eta_{! *} M_{\eta}\right)=0$.

Proof: There is some $j: U \subset \operatorname{Spec} \mathcal{O}_{F}$ and a smooth Artin-Tate motive $M \in$ $\operatorname{MATM}(U)=\mathbf{M M}(U) \cap \mathbf{D A T M}(U)$ such that $M_{\eta}=\eta^{*}[-1] M$. Shrinking $U$ further (using $j_{!_{*}}^{\prime} j^{\prime *} M \cong M$ for some $j^{\prime}: U^{\prime} \subset U$, as $M$ is smooth), we may assume by the standard splitting routine [Sch11, Lemma 2.5] that there is an etale Galois cover $f^{\prime}: V^{\prime} \rightarrow U$ such that $f^{\prime *} M$ is a mixed Tate motive over $V^{\prime}$. The $\operatorname{map} M \rightarrow f_{*}^{\prime} f^{\prime *} M \stackrel{\cong}{\cong} f_{!}^{\prime} f^{\prime !} M \rightarrow M$ is $\operatorname{deg} f^{\prime} \cdot \operatorname{id}_{M}$, so $M$ is a direct summand of $f_{*}^{\prime} f^{\prime *} M$, since we use rational coefficients. The functor $f_{*}^{\prime}=f_{!}^{\prime}$ preserves ArtinTate motives and is exact [Sch11, Theorem 4.2]. Hence $j_{!_{*}} f_{*}^{\prime} f^{\prime *} M=f_{*} j_{!_{*}}^{\prime} f^{\prime *} M$. 
Here $f: V \rightarrow$ Spec $\mathcal{O}_{F}$ is the normalization of $\mathcal{O}_{F}$ in the function field of $V^{\prime}$ and $j^{\prime}: V^{\prime} \rightarrow V$ is the corresponding open immersion. Consequently,

$\mathrm{H}^{3}\left(\mathcal{O}_{F}, \eta_{!_{*}} M_{\eta}\right)=\mathrm{H}^{3}\left(\mathcal{O}_{F}, j_{!_{*}} M\right) \subset \mathrm{H}^{3}\left(V, j_{!_{*}}^{\prime} f^{\prime *} M\right)=\operatorname{Hom}_{V}\left(j_{!_{*}}^{\prime} f^{\prime *} M,(\mathbf{1}[1])[2]\right)=0$, since the cohomological dimension of mixed Tate motives over $V$ is one, as opposed to two for Artin-Tate motives [Sch11, Proposition 4.4].

The following conjecture will be needed to deal with motives over $\mathbb{F}_{p}$.

Conjecture 1.5. (Beilinson) Let $X / \mathbb{F}_{q}$ be smooth and projective. Up to torsion, numerical and rational equivalence agree on $X$.

Recall that homological equivalence lies between these two equivalence relations And04, 3.2.1], so under [1.5, all three agree. The second important consequence of 1.5 is that the category of pure Chow motives over $\mathbb{F}_{q}$ is semisimple by Jannsen's theorem.

To study $L$-functions, we need some $\ell$-adic realization functor. We use the machinery developed recently by Ayoub Ayo12. It allows the base scheme to be $\mathbb{Z}[1 / \ell]$. Let $\ell$ be an odd prime number and $S$ a scheme that is of finite type over $\mathbb{Z}$ or $\mathbb{Q}$ such that $\ell$ is invertible on $S$ (cf. Ayo12, Hyp. 6.5]). Define the $\ell$-adic realization functor as the following composition

$$
\begin{aligned}
(-)_{\ell}: \mathbf{D M}_{\mathrm{b}}(S) \stackrel{F_{7}}{\rightarrow} \mathbf{S H}(S)_{\mathbb{Q}} & \stackrel{F_{2}}{\rightarrow} D A^{\text {ét }}\left(S, \mathbb{Q}_{\ell}\right) \\
& \stackrel{R_{\ell}}{\rightarrow} \mathbf{D}\left(\mathbf{S h v}_{\text {ét }}\left(S, \mathbb{Q}_{\ell}\right)\right) \stackrel{F_{3}}{\longrightarrow} \mathbf{D}\left(\mathbf{S h v}_{\text {ét }}\left(S, \mathbb{Q}_{\ell}\right)\right)
\end{aligned}
$$

The functor $F_{1}$ is the inclusion of the category of $\mathbf{1}$-modules in $\mathbf{S H}(S)_{\mathbb{Q}}$. The category $D A^{\text {ét }}\left(S, \mathbb{Q}_{\ell}\right)$ is the homotopy category of the model category of symmetric $\mathbb{P}^{1}$ spectra of complexes of $\ell$-adic presheaves on $\mathbf{S m} / S$, endowed with the $\mathbb{A}^{1}$-étale-local model structure. The functor $F_{2}$ is obtained by combining the natural free abelian group functor $\Delta^{\mathrm{op}}$ Sets $\rightarrow \mathbf{C o m}(\mathbf{A b})$ and the sheafification (from Nisnevich sheaves to etale sheaves), see e.g. [CD09, 5.3.28, 5.3.37]. The functor $R_{\ell}$ is Ayoub's $\ell$-adic realization functor. We append the contravariant functor $F_{3}: M \mapsto \underline{\operatorname{Hom}}\left(M, f^{!} \mathbb{Q}_{\ell}\right)$, where $f: S \rightarrow$ Spec $\mathbb{Z}$ is the structural map (and Hom denotes the derived inner Hom). For any map $g: X \rightarrow Y$ of quasi-projective $S$-schemes, the functors $F_{1}$, $F_{2}, R_{\ell}$ commute with $g_{!}, g_{*}, g^{*}$ and $g^{!}$and, when applied to compact objects, with

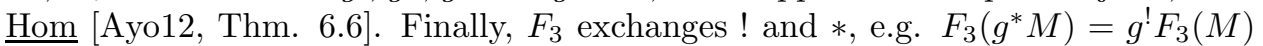
for $M \in \mathbf{D}\left(\mathbf{S h v}_{\text {ét }}\left(S, \mathbb{Q}_{\ell}\right)\right)$. Therefore, for some quasi-projective scheme $f: X \rightarrow S$, $(\mathrm{M}(X)(-m)[-n])_{\ell}=f_{*} f^{*} \mathbb{Q}_{\ell}(m)[n]$. This property is also satisfied for Huber's and Ivorra's realization functors provided $S$ is a field [Hub00, Ivo07. Thus, for the mere definition in 3.1, these realization functors are sufficient, but Lemma 3.4 relies on a realization functor over $\mathbb{Z}[1 / \ell]$.

The exactness requirement for the functor $-\ell$ mentioned in Axiom 1.2(ii) means that the restriction of $-\ell$ to $\mathbf{D M}_{\mathrm{B}, c}(S)$ is exact with respect to the (conjectural) motivic $t$-structure and the $t$-structure on $\mathbf{D}\left(\mathbf{S h v}_{\text {ét }}\left(S, \mathbb{Q}_{\ell}\right)\right.$ ) (which is the obvious one if $S$ is a field and the perverse $t$-structure for $S=$ Spec $\mathbb{Z}[1 / \ell]$, see $[\mathrm{Sch} 12 \mathrm{~b}$, Section 3]. For example, for a quasi-projective variety $X$ over a field it implies

$$
\left(\mathrm{h}^{-b}(X)\right)_{\ell}=\mathrm{H}^{b}\left(X, \mathbb{Q}_{\ell}\right) .
$$

\section{Arakelov motivic cohomology}

\subsection{Deligne cohomology}

A key input to Beilinson's conjecture 5.14 is Deligne cohomology. We recall its classical definition and the well-known interpretation in terms of weak Hodge cohomology. Then, we recall from HS11 the Deligne cohomology spectrum $\mathrm{H}_{\mathrm{D}}$ which 
is crucial for the definition of Arakelov motivic cohomology. In order to establish the $\mathbb{Q}$-structure on the groups represented by $\mathrm{H}_{\mathrm{D}}$, we explain how to apply the construction in loc. cit. to obtain spectra representing Betti and de Rham cohomology.

Let an : $\mathbf{S m} / \mathbb{C} \rightarrow \mathbf{S m}^{\text {an }}$ be the functor that associates to any smooth $\mathbb{C}$-scheme the underlying complex analytic manifold. We also consider an : $\mathbf{S m} / \mathbb{Q}($ or $\mathbf{S m} / \mathbb{R}) \rightarrow \mathbf{S m}^{\text {an }, G}$, where the target category consists of complex analytic manifolds with a $G$-action, $G:=\operatorname{Gal}(\mathbb{C} / \mathbb{R})$. In this section, $X$ is a smooth scheme over $\mathbb{Q}$. We usually write $X^{\text {an }}:=\operatorname{an}(X)$ and $\operatorname{Fr}_{\infty}: X^{\text {an }} \rightarrow X^{\text {an }}$ for the conjugation. We also pick a smooth proper compactification $j: X \rightarrow \bar{X}$ (over $\mathbb{Q}$ ) such that $D:=\bar{X} \backslash X$ is a divisor with strict normal crossings. We write $\Omega_{X^{*}}^{*}\left(\log D^{\text {an }}\right)$ for the complex of meromorphic forms on $\bar{X}$ that are holomorphic on $X \subset \bar{X}$, and have at worst logarithmic poles at the divisor $D$. This complex is endowed with the Hodge filtration $F^{p}:=\sigma_{\geq p}$, which is simply the brutal truncation. The variant using algebraic (i.e., Kähler) differential forms is denoted $\Omega_{\frac{*}{X}}^{* \text { alg }}(\log D)$. The $C^{\infty}$-variant is denoted $E_{\bar{X}^{\text {an }}}^{*}\left(\log D^{\text {an }}\right)$. The subspace of real-valued forms is denoted $E_{\mathbb{R}, \bar{X}^{\text {an }}}^{*}\left(\log D^{\text {an }}\right)$. These complexes are filtered by $F^{p} E_{\bar{X}^{\text {an }}}^{n}\left(\log D^{\text {an }}\right)=\oplus_{a+b=n, a \geq p} E_{\bar{X}^{a n}}^{a, b}\left(\log D^{\text {an }}\right)$. To get rid of the choice of $\bar{X}$, put

$$
E^{*}(X):=\underset{\vec{X}}{\lim _{\bar{X}}} E^{*}\left(\log D^{\text {an }}\right),
$$

and similarly for $E_{\mathbb{R}}^{*}(X), \Omega^{*}(X), \Omega^{*, a l g}(X)$. Here, the colimit runs over the directed category of all compactifications $\bar{X}$ as above. Finally, let $\mathbb{R}(p):=(2 \pi i)^{p} \mathbb{R} \subset \mathbb{C}$ be the constant sheaf.

Definition 2.1. Set $\mathbb{R}_{\mathrm{D}, D, \bar{X}}(p):=\operatorname{cone}\left(\mathrm{R} j_{*} \mathbb{R}(p) \oplus F^{p} \Omega_{\bar{X}^{*}}\left(\log D^{\text {an }}\right) \rightarrow \mathrm{R} j_{*} \Omega_{X^{\text {an }}}^{*}\right)$. For example, if $X$ is proper, $\mathbb{R}_{\mathrm{D}}(p) \cong\left[\mathbb{R}(p) \rightarrow \Omega_{X^{\text {an }}}^{0} \rightarrow \ldots \rightarrow \Omega_{X^{\text {an }}}^{p-1}\right]$, with the terms lying in degrees 0 to $p$. Deligne cohomology of $X$ is defined as the $G$-invariant subspace of a sheaf hypercohomology group,

$$
\mathrm{H}_{\mathrm{D}}^{n}(X, p):=\mathbb{H}^{n}\left(\bar{X}^{\mathrm{an}}, \mathbb{R}_{\mathrm{D}, D, \bar{X}}(p)\right)^{G} .
$$

(The $G$-action is obtained by letting $G$ act on $\mathbb{R}(p)$ as $a \mapsto \overline{\mathrm{Fr}_{\infty}^{*}(a)}$ and on $\Omega^{*}$ by $\omega \mapsto \operatorname{Fr}_{\infty}^{*}(\omega)$. This group does not depend on the choice of $\bar{X}$ [EV88, Lemma 2.8].)

By definition, there is a long exact sequence

$$
\ldots \rightarrow\left(\mathrm{H}_{\mathrm{dR}}^{i}\left(X^{\mathrm{an}}\right) / F^{p} \mathrm{H}^{i}\left(X^{\mathrm{an}}, \Omega_{X}^{*}\right)^{G} \rightarrow \mathrm{H}_{\mathrm{D}}^{i+1}(X, m) \rightarrow \mathrm{H}^{i+1}\left(X^{\mathrm{an}}, \mathbb{R}(m)\right)^{(-1)^{m}} \rightarrow \ldots\right.
$$

Here the superscript denotes the $(-1)^{m}$-eigenspace of the $\mathrm{Fr}_{\infty}$-action on Betti cohomology of $X^{\text {an }}$. This sequence induces an isomorphism

$$
\operatorname{det} \mathrm{H}_{\mathrm{D}}^{*}(X, m)=\operatorname{det}^{-1}\left(\mathrm{H}_{\mathrm{dR}}^{*}\left(X^{\mathrm{an}}\right) / F^{m}\right)^{G} \otimes \operatorname{det} \mathrm{H}^{*}\left(X^{\mathrm{an}}, \mathbb{R}(m)\right)^{(-1)^{m}} .
$$

The right hand side carries a natural $\mathbb{Q}$-structure stemming from the isomorphisms $\mathrm{H}^{*}\left(X^{\text {an }}, \mathbb{R}(m)\right)=\mathrm{H}^{*}\left(X^{\text {an }}, \mathbb{Q}(m)\right) \otimes_{\mathbb{Q}} \mathbb{R}$ and $\mathrm{H}^{*}\left(\bar{X}^{\mathrm{an}}, F^{*} \Omega_{\bar{X}^{*}}^{\text {an }}\left(\log D^{\text {an }}\right)\right)^{G} \cong$

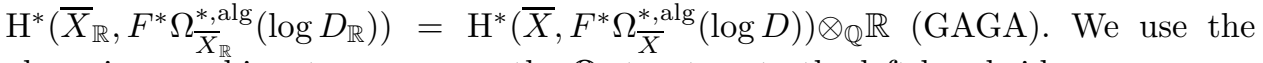
above isomorphism to carry over the $\mathbb{Q}$-structure to the left hand side.

If $X$ is (smooth and) proper, the degeneration of the Hodge-de Rham spectral sequence and weight reasons give us short exact sequences (loc. cit.)

$$
0 \rightarrow \mathrm{H}^{i}\left(X^{\mathrm{an}}, \mathbb{R}(m)\right)^{(-1)^{m}} \rightarrow \mathrm{H}_{\mathrm{dR}}^{i}\left(X_{\mathbb{R}}\right) / F^{m} \rightarrow \mathrm{H}_{\mathrm{D}}^{i+1}(X, m) \rightarrow 0
$$

for $i-2 m \leq-2$ and, for $i-2 m \geq 0$,

$$
0 \rightarrow \mathrm{H}_{\mathrm{D}}^{i}(X, m) \rightarrow \mathrm{H}^{i}\left(X^{\mathrm{an}}, \mathbb{R}(m)\right)^{(-1)^{m}} \rightarrow \mathrm{H}_{\mathrm{dR}}^{i}\left(X_{\mathbb{R}}\right) / F^{m} \rightarrow 0,
$$


respectively. In this case, each individual Deligne cohomology group carries a $\mathbb{Q}$ structure, as opposed to the general case of a merely smooth $X / \mathbb{Q}$.

Now, we recall Beilinson's notion of weak absolute Hodge cohomology. It is relevant to us because of its relation to archimedean factors of $L$-functions, see (3.2). It is based on Deligne's abelian category $\mathbf{M H S}_{\mathbb{Q}}(\mathbb{R})$ of mixed Hodge structures Del71, 2.3.1]. The subscript $\mathbb{Q}$ indicates that we are considering $\mathbb{Q}$-vector spaces, " $(\mathbb{R})$ " means that the structure is endowed with an action of $G=\operatorname{Gal}(\mathbb{C} / \mathbb{R})$. For example, $\mathbf{1}(n)$ is the one-dimensional $\mathbb{Q}$-space, such that it is pure of weight $-2 n$ and the Hodge filtration is concentrated in degree $-n$, and the non-trivial element of $G$ acts as multiplication by $(-1)^{n}$. Let

$$
\mathbf{C o m}_{\mathrm{H}}^{\mathrm{b}}=\left\{C=\left(C_{\mathrm{dR}}, C_{\mathrm{B}}, C_{c}, i_{\mathrm{dR}}, i_{\mathrm{B}}\right)\right\}
$$

be the category of bounded Hodge complexes [Ber86, 3.2]. Its objects consist of a bounded bifiltered complex of $\mathbb{Q}$-vector spaces $\left(C_{\mathrm{dR}}, W_{*}, F^{*}\right)$, a filtered complex of $\mathbb{Q}[G]$-modules $\left(C_{\mathrm{B}}, W_{*}\right)$ and a filtered complex of $\mathbb{C}$-modules with $\mathbb{C}$-antilinear $G$ action, $\left(C_{c}, W_{*}\right)$, a filtered $G$-equivariant quasi-isomorphism $i_{\mathrm{B}}:\left(C_{\mathrm{B}}, W_{*}\right) \otimes_{\mathbb{Q}} \mathbb{C} \rightarrow$ $\left(C_{c}, W_{*}\right)\left(G\right.$ acts on the left hand term by the action on $C_{\mathrm{B}}$ and complex conjugation on $\mathbb{C})$ and finally a filtered $G$-equivariant quasi-isomorphism $i_{\mathrm{dR}}:\left(C_{\mathrm{dR}}, W_{*}\right) \otimes_{\mathbb{Q}} \mathbb{C} \rightarrow$ $\left(C_{c}, W_{*}\right)$ (on the left, $G$ acts by conjugation on $\mathbb{C}$ ). These data are subject to the requirement that the cohomology quintuple $\mathrm{H}^{i}(C)$ defined by the cohomologies of the various complexes and comparison maps has to be an object of $\mathbf{M H \mathbf { S } _ { \mathbb { Q } }}(\mathbb{R})$. Morphisms in the category $\mathbf{C o m}_{\mathrm{H}}^{\mathrm{b}}$ are required to respect the filtrations and the comparison quasi-isomorphisms. To any Hodge complex, we can associate its weak Hodge cohomology [Beř66, 3.13]

$$
\mathrm{R} \Gamma_{\mathrm{w}}(C):=\operatorname{cone}[-1]\left(C_{\mathrm{B}}^{G} \otimes \mathbb{R} \oplus F^{0} C_{\mathrm{dR}} \otimes \mathbb{R}^{i_{\mathrm{B}}-i_{\mathrm{d}}} C_{c}^{G}\right) \in \mathbf{C o m}(\underline{\mathbb{R}}) .
$$

This descends to a functor

$$
\mathrm{R} \Gamma_{\mathrm{w}}: \mathbf{D}_{\mathrm{H}}^{\mathrm{b}}:=\mathbf{C o m}_{\mathrm{H}}^{\mathrm{b}} / \text { quasi-isomorphisms } \rightarrow \mathbf{D}^{\mathrm{b}}(\underline{\mathbb{R}})^{\mathbb{Q}-\operatorname{det}} .
$$

Indeed, taking $G$-invariants and applying the Hodge filtration are exact operations, since morphisms of Hodge structures respect the Hodge filtration strictly Del71, 2.3.5(iii)]. The $\mathbb{Q}$-structure on $\mathrm{R}_{\mathrm{w}}(C)$ is the one stemming from the very definition, where $C_{c}^{G}$ is endowed with a $\mathbb{Q}$-structure using the one on $C_{\mathrm{dR}}$ via $i_{\mathrm{dR}}$. Set $\mathrm{H}_{\mathrm{w}}^{i}(C):=$ $\mathrm{H}^{i}\left(\mathrm{R}_{\mathrm{w}}(C)\right)$. A spectral sequence argument yields an exact sequence:

$$
0 \rightarrow \mathrm{H}_{\mathrm{w}}^{1}\left(\mathrm{H}^{i-1} C\right) \rightarrow \mathrm{H}_{\mathrm{w}}^{i}(C) \rightarrow \mathrm{H}_{\mathrm{w}}^{0}\left(\mathrm{H}^{i} C\right) \rightarrow 0 .
$$

Unlike absolute Hodge cohomology, i.e., the derived functor of $V \mapsto \Gamma_{\text {MHS }}(V):=$ $\operatorname{Hom}_{\mathrm{MHS}}(\mathbf{1}, V)=\mathrm{H}_{\mathrm{w}}^{0}\left(W_{0} V\right)$, the weak variant has a duality: the natural pairing (induced by $A \times A^{\vee} \rightarrow \mathbb{R}$ for any $\mathbb{R}$-vector space $A$ ),

$$
\mathrm{H}_{\mathrm{w}}^{i}(C) \times \mathrm{H}_{\mathrm{w}}^{1-i}\left(C^{\vee}(1)\right) \rightarrow \mathrm{H}_{\mathrm{w}}^{1}(\mathbf{1}(1))=\mathbb{R},
$$

is perfect for all $i$ FPR94, Prop.III.1.2.3].

The following well-known lemma states that weak Hodge cohomology is the same as Deligne cohomology. Recall the Hodge complex $\underline{\mathrm{R}}(X, m)$ of [Bei86, Section 4] whose cohomology objects are the Hodge structures $\mathrm{H}^{i}\left(X^{\mathrm{an}}, \mathbb{Q}(m)\right)$.

Lemma 2.2. For $X / \mathbb{Q}$ smooth and projective and any $i, m$, we have

$$
\mathrm{H}_{\mathrm{w}}^{i}(\underline{\mathrm{R} \Gamma}(X, m))=\mathrm{H}_{\mathrm{D}}^{i}(X, m) .
$$

The induced isomorphism $\operatorname{det} \mathrm{H}_{\mathrm{w}}^{*}(\underline{\mathrm{R}}(X, m))=\operatorname{det} \mathrm{H}_{\mathrm{D}}^{*}(X, m)$ respects the $\mathbb{Q}$ structure. 
Proof: The Hodge structures $L_{i}:=\mathrm{H}^{i}(\underline{\mathrm{R}} \Gamma(X, m))=\mathrm{H}^{i}\left(X^{\text {an }}, \mathbb{Q}(m)\right)$ are pure of weight $i-2 m$. For $i-2 m<0, \mathrm{H}_{\mathrm{w}}^{0}\left(L_{i}\right)=\Gamma_{\mathrm{MHS}}\left(L_{i}\right)=0$. By duality, $\mathrm{H}_{\mathrm{w}}^{1}\left(L_{i}\right)=$ $\mathrm{H}_{\mathrm{w}}^{0}\left(L_{i}^{\vee}(1)\right)^{\vee}=0$ for $i-2 m>-2$. Hence, by (2.4),

$$
\mathrm{H}_{\mathrm{w}}^{i} \underline{\mathrm{R}}(X, m)= \begin{cases}\mathrm{H}_{\mathrm{w}}^{1}\left(L_{i-1}\right) & i-2 m<0 \\ \mathrm{H}_{\mathrm{w}}^{0}\left(L_{i}\right) & i-2 m \geq 0\end{cases}
$$

The map in the exact sequences (2.2) between Betti and de Rham cohomology is the one from the definition of $\mathrm{R} \Gamma_{\mathrm{w}}\left(L_{*}\right)$. This shows (2.6). The identification of the $\mathbb{Q}$-structures follows similarly.

For archimedean factors of $L$-functions of arbitrary motives, we use the Hodge realization functor (see [Beí86, Section 3] for an early avatar):

$$
\mathrm{R} \Gamma_{\mathrm{H}}: \mathbf{D M}_{\mathrm{B}, c}(\mathbb{Q})^{\mathrm{op}} \stackrel{\cong,(1.2)}{\longrightarrow} \mathbf{D M}_{\mathrm{gm}}(\mathbb{Q})^{\mathrm{op}} \rightarrow \mathbf{D}_{\mathrm{H}}^{\mathrm{b}} .
$$

The right hand functor is Huber's Hodge realization functor Hub00, 2.3.5]. It maps $\mathrm{M}_{\mathrm{gm}}(X)(-m)$ to $\underline{\mathrm{R}}(X, m)$. For any $M \in \mathbf{D M}_{\mathrm{b}, c}(\mathbb{Q})$, the natural map $\mathrm{R} \Gamma_{\mathrm{H}}(\underline{\operatorname{Hom}}(M, \mathbf{1})) \rightarrow \underline{\operatorname{Hom}}\left(\mathrm{R} \Gamma_{\mathrm{H}}(M), \mathrm{R} \Gamma_{\mathrm{H}}(\mathbf{1})\right)$ is an isomorphism. It is enough to check this on generators $M=\mathrm{M}(X)$ with $X / \mathbb{Q}$ smooth and projective, where it follows from $(\mathrm{M}(X))^{\vee}=\mathrm{M}(X)\{\operatorname{dim} X\}$. We obtain $\mathrm{R} \Gamma_{\mathrm{H}}\left(M^{\vee}(1)\right)=\left(\mathrm{R} \Gamma_{\mathrm{H}}(M)\right)^{\vee}(-1)$. We put

$$
\mathrm{R} \Gamma_{\mathrm{wH}}:=\mathrm{R} \Gamma_{\mathrm{w}} \circ \mathrm{R} \Gamma_{\mathrm{H}}: \mathbf{D M}_{\mathrm{b}, c}(\mathbb{Q}) \rightarrow \mathbf{D}^{\mathrm{b}}(\underline{\mathbb{R}})^{\mathbb{Q}-\operatorname{det}} .
$$

The composition of these functors with $\eta^{*}: \mathbf{D M}_{\mathrm{b}, c}(\mathbb{Z}) \rightarrow \mathbf{D M}_{\mathrm{b}, c}(\mathbb{Q})$ will be denoted the same.

Finally, we recall the construction of the Deligne cohomology spectrum $\mathrm{H}_{\mathrm{D}}$ HS11. We also sketch how to obtain similar spectra for Betti and de Rham cohomology. The aim is (2.13), the $\mathbb{Q}$-structure on Deligne cohomology groups of general motives.

Let $\mathcal{C}$ be either the category $\mathbf{S m}^{G \text {,an }}$ or $\mathbf{S m} / \mathbb{Q}$. Consider simplicial presheaves $C(p)$ of pointed sets on $\mathcal{C}$, for each $p \geq 0$, together with a "product" map $\cdot_{C}: C(p) \wedge$ $C\left(p^{\prime}\right) \rightarrow C\left(p+p^{\prime}\right)$. Moreover, we assume there is an element $c_{1} \in C(1)\left(\mathbb{G}_{m}\right)$, that restricts to zero at the point $1 \in \mathbb{G}_{m}$ (equivalently, a pointed map $c_{1}:\left(\mathbb{G}_{m}, 1\right) \rightarrow$ $C(1))$ such that for any two maps $f_{i}: U \rightarrow \mathbb{G}_{m}, U \in \mathcal{C}, i=1,2$,

$$
f_{1}^{*}\left(c_{1}\right) \cdot{ }_{C}\left(f_{2}^{*}\left(c_{1}\right) \cdot C c^{\prime}\right)=f_{2}^{*}\left(c_{1}\right) \cdot C\left(f_{1}^{*}\left(c_{1}\right) \cdot{ }_{C} c^{\prime}\right) .
$$

The element $c_{1}$ is referred to as a bonding element. Under these assumptions, the presheaves $C(p)$ with the bonding maps $\mathbb{G}_{m} \wedge C(p) \stackrel{c_{1} \wedge \text { id }}{\longrightarrow} C(1) \wedge C(p) \stackrel{{ }^{C}}{\longrightarrow} C(p+1)$ form a symmetric $\mathbb{G}_{m}$-spectrum $C$ (where the $\Sigma_{p}$-action on $C(p)$ is trivial). The category of such spectra is denoted $\operatorname{Spt}(\mathcal{C})$. It is endowed with a model structure whose homotopy category $\mathbf{S H}(\mathbb{Q})$ (or $\mathbf{S H}\left(\mathbb{R}^{\text {an }}\right)$ ) satisfies (cf. e.g. Ayo10, Section 1] for the analytic version):

$$
\operatorname{Hom}_{\mathbf{S H}}\left(\Sigma^{\infty}(X \sqcup\{*\}) \wedge S^{n} \wedge \mathbb{G}_{m}^{\wedge m}, C\right)=\pi_{n+m+N}(C(m+N)(X))
$$

for any $X \in \mathcal{C}$, and $n, m \in \mathbb{Z}$ and $N \gg 0$, provided that

1. all levels $C(p)$ are homotopy invariant: $C(p)(-) \rightarrow C(p)\left(-\times \mathbb{A}^{1}\right)$ (respectively, $\left.-\times\left(\mathbb{A}^{1}\right)^{\text {an }}\right)$ is a weak equivalence,

2. all levels $C(p)$ satisfy descent (with respect to the Nisnevich and the analytic topology, respectively), and 
3. $C$ is an $\Omega$-spectrum. In the presence of the first two conditions, this is implied by the bundle formula, which says that

$$
\oplus_{i=0}^{1} p_{X}^{*}(-) \cdot C p_{\mathbb{G}_{m}}^{*}\left(c_{1}\right)^{i}: \oplus \pi_{i+*}(C(p-i)(X)) \rightarrow \pi_{*}\left(C(p)\left(X \times \mathbb{G}_{m}\right)\right)
$$

is an isomorphism, where $p_{X}, p_{\mathbb{G}_{m}}: X \times \mathbb{G}_{m} \rightarrow X, \mathbb{G}_{m}$ are the projections.

The spectra below are all obtained by putting $C(p):=D K(\tau \geq 0 A(p))$ for appropriate complexes of abelian groups $A(p)$. Here $\tau$ is the good truncation and $D K$ the DoldKan equivalence.

We now define four different (but isomorphic) spectra representing Betti cohomology with real coefficients by specifying the levels $C(p)$ and the bonding elements in $C(1)\left(\mathbb{G}_{m}\right)$. The product structure map on the level complexes is obvious for these Betti cohomology spectra, and is strictly commutative and associative. For any presheaf of abelian groups $F$ on $\mathbf{S m}^{G \text {, an }}$, we define the Čech-complex in degrees $n \geq 0$

$$
\mathfrak{C}^{n} F(X):=\lim _{\longleftarrow} F\left(U^{n+1}\right)
$$

The limit runs over the directed category of all open covers $\left\{U_{i}\right\}$ of $X \in \mathbf{S m}^{G \text {,an }}$ and $U:=\sqcup U_{i}$. Given some involution ? $: F \rightarrow F$, we write $\mathfrak{C}^{G} F$ for the subcomplex consisting of elements that are fixed by $\overline{\mathrm{Fr}_{\infty}^{*}}$.

Let $\mathrm{H}_{\mathrm{B}, \mathbb{R}}^{(1)}$ be the spectrum whose levels are $\mathfrak{C}^{G}(\mathbb{R}(p)[p])$. To describe the bonding element, we replace $\mathbb{G}_{m}^{\text {an }}$ by $S^{1}$ (equipped with its usual topology). The inclusion $S^{1} \subset \mathbb{G}_{m}^{\text {an }}$ is a homotopy equivalence, and an explicit description of a Čech cocycle generating $\mathrm{H}^{1}\left(\mathbb{G}_{m}^{\text {an }}, \mathbb{C}\right)$ is left to the reader. As for $S^{1}$, consider the standard covering by $U_{ \pm}=\left\{z \in S^{1}, \pm \Re(z)>-0.5\right\}$. This covering is equivariant with respect to $z \mapsto \bar{z}$. Frobenius $\operatorname{Fr}_{\infty}$ acts on the Cech complex

$$
\mathbb{R}(1)\left(U_{+}\right) \oplus \mathbb{R}(1)\left(U_{-}\right) \rightarrow \mathbb{R}(1)\left(U_{+} \cap U_{-}\right)=\mathbb{R}(1)^{2},(a, b) \mapsto(v, w):=(b-a, b-a)
$$

as $(a, b) \mapsto(a, b)$ and $(v, w) \mapsto(w, v)$. Hence $(\pi i,-\pi i) \in \mathbb{R}(1)\left(U_{+} \cap U_{-}\right)$is a $\overline{\operatorname{Fr}_{\infty}}$ invariant element which generates $\mathrm{H}^{1}\left(\mathbb{G}_{m}^{\text {an }}, \mathbb{R}(1)\right)^{G}$. This determines the spectrum $\mathrm{H}_{\mathrm{B}, \mathbb{R}}^{(1)}$. It is well-known that $\mathrm{H}^{*}\left(\mathfrak{C}^{*}(\mathbb{R})(X)\right)=\mathrm{H}^{*}(X, \mathbb{R})$. Thus

$$
\operatorname{Hom}_{\mathbf{S H}\left(\mathbb{R}^{\text {an }}\right)}\left(\Sigma^{\infty} X, \mathrm{H}_{\mathrm{B}, \mathbb{R}}^{(1)}(p)[n]\right)=\mathrm{H}_{\mathrm{B}}^{n}(X, \mathbb{R}(p))^{(-1)^{p}},
$$

where the superscript at the right denotes the subgroup of elements $a$ satisfying $\operatorname{Fr}_{\infty}^{*}(a)=(-1)^{p} a$. The complexes $\operatorname{Tot}\left(\mathfrak{C}^{G}\left(E_{\mathbb{R}}^{*}(p)[p]\right)\right)$ and the bonding element induced by the previous one via the inclusion $\mathbb{R}(1)[1] \subset E_{\mathbb{R}}^{0}(1)[1]$ yield a spectrum $\mathrm{H}_{\mathrm{B}, \mathbb{R}}^{(2)}$ that is naturally isomorphic to $\mathrm{H}_{\mathrm{B}, \mathbb{R}}^{(1)}$, since $\mathbb{R} \rightarrow E_{\mathbb{R}}^{*}$ is a quasi-isomorphism of sheaf complexes. Consider the spectrum $\mathrm{H}_{\mathrm{B}, \mathbb{R}}^{(3)}$ whose levels are the one of $\mathrm{H}_{\mathrm{B}, \mathbb{R}}^{(2)}$, but the bonding element is the 1 -form

$$
d z / z \in E_{\mathbb{R}, \mathbb{P}^{1}}^{1}(\log \{0, \infty\}) \rightarrow \mathfrak{C}^{0} E_{\mathbb{R}}^{1}(1)\left(\mathbb{G}_{m}^{\text {an }}\right) \subset \operatorname{Tot}\left(\mathfrak{C}^{*} E_{\mathbb{R}}^{*}(1)\right)^{1}\left(\mathbb{G}_{m}^{\text {an }}\right) .
$$

Both $\mathrm{H}_{\mathrm{B}, \mathbb{R}}^{(2)}$ and $\mathrm{H}_{\mathrm{B}, \mathbb{R}}^{(3)}$ are $\Omega$-spectra (the above bonding element and $d z / z$ give the same element in $\mathrm{H}^{1}\left(\mathbb{G}_{m}^{\mathrm{an}}, \mathbb{R}(1)\right)$ by Cauchy's residue formula). The identity map between their level-0-complexes thus yields a canonical isomorphism of spectra (in $\left.\mathbf{S H}\left(\mathbb{R}^{\mathrm{an}}\right)\right)$. The complexes $E_{\mathbb{R}}^{*, G}(p)[p]$ (again ${ }^{G}$ denotes invariants under $\overline{\operatorname{Fr}_{\infty}^{*}}$ ) together with the bonding element $d z / z$ form a spectrum denoted $\mathrm{H}_{\mathrm{B}, \mathbb{R}}^{(4)}$. The obvious quasi-isomorphism $E_{\mathbb{R}}^{*, G}=\mathfrak{C}^{0, G} E_{\mathbb{R}}^{*} \rightarrow \operatorname{Tot}\left(\mathfrak{C}^{G} E_{\mathbb{R}}^{*}\right)$ induces an isomorphism $\mathrm{H}_{\mathrm{B}, \mathbb{R}}^{(4)} \rightarrow$ $\mathrm{H}_{\mathrm{B}, \mathbb{R}}^{(3)}$ in $\mathbf{S H}\left(\mathbb{R}^{\text {an }}\right)$. The purpose of the chain of isomorphisms $\mathrm{H}_{\mathrm{B}, \mathbb{R}}^{(4)} \cong \mathrm{H}_{\mathrm{B}, \mathbb{R}}^{(1)}$ is the existence of $\mathrm{H}_{\mathrm{B}, \mathbb{Q}}^{(1)}$, the obvious $\mathbb{Q}$-linear variant of $\mathrm{H}_{\mathrm{B}, \mathbb{R}}^{(1)}$. It induces a $\mathbb{Q}$-structure on the groups represented by $\mathrm{H}_{\mathrm{B}, \mathbb{R}}^{(4)}$. 
As for de Rham cohomology, consider the complexes $E^{F}(p)^{G}:=\operatorname{cone}\left(F^{p} E^{*} \rightarrow\right.$ $\left.E^{*}\right)^{G}[p-1]$. The product

$$
\begin{aligned}
\left(F^{p} E^{n} \oplus E^{n-1}\right) \otimes\left(F^{p^{\prime}} E^{n^{\prime}} \oplus E^{n^{\prime}-1}\right) & \rightarrow\left(F^{p+p^{\prime}} E^{n+n^{\prime}} \oplus E^{n+n^{\prime}-1}\right), \\
\left(f_{1}, e_{1}\right) \otimes\left(f_{2}, e_{2}\right) & \mapsto\left(f_{1} \wedge f_{2}, f_{1} \wedge e_{2}\right)
\end{aligned}
$$

is strictly associative, but in general commutative only up to homotopy EV88, Section 3]. However, putting $c_{1}=(d z / z, 0) \in E^{F}(1)\left(\mathbb{G}_{m}\right)=\left(F^{1} E^{1} \oplus E^{0}\right)\left(\mathbb{G}_{m}\right)$, (2.9) clearly holds. We obtain a spectrum $\mathrm{H}_{\mathrm{dR}}^{/ F \text {,an }} \in \mathbf{S H}\left(\mathbb{R}^{\text {an }}\right)$. Using $\operatorname{Fr}_{\infty}^{*}$-invariant algebraic differential forms, i.e., $\Omega^{* \text {,alg, } G}$ instead of $E^{*, G}$, we get a similar spectrum denoted $\mathrm{H}_{\mathrm{dR}}^{/ F \text {,alg }} \in \mathbf{S H}(\mathbb{Q})$. For smooth $X / \mathbb{Q}$, the obvious maps

$$
\Omega^{*, \text { alg }}(X) \otimes_{\mathbb{Q}} \mathbb{R} \rightarrow \Omega^{*, \text { alg }}\left(X_{\mathbb{R}}\right)=\Omega^{*, \text { alg }, G}\left(X_{\mathbb{C}}\right) \leftarrow \Omega^{*, G}(X) \rightarrow E^{*, G}(X)
$$

are filtered (with respect to the Hodge filtration) quasi-isomorphisms by flat base change for $\Omega^{*, \text { alg }}$, GAGA and [Bur94, Thm. 2.1]. We thus get an isomorphism $c_{*} \mathrm{an}_{*} \mathrm{H}_{\mathrm{dR}}^{/ F, \text { an }}=\mathrm{H}_{\mathrm{dR}}^{/ F, \text { alg }} \otimes \mathbb{R}$ in $\mathbf{S H}(\mathbb{Q})$. Here $c:$ Spec $\mathbb{R} \rightarrow$ Spec $\mathbb{Q}$.

Finally, the complex

$$
\mathrm{D}(p)^{G}:=\operatorname{cone}\left(E_{\mathbb{R}}^{*, G}(p)[p] \rightarrow E^{F}(p)^{G}\right)[-1]
$$

carries a product map ${ }^{\mathrm{D}, \alpha}$ depending on some auxiliary parameter $\alpha \in \mathbb{R}$. It is only commutative and associative up to homotopy (for each $\alpha$ ). Again, $c_{1}=$ $(d z / z, d z / z, 0) \in \mathrm{D}(1)^{0}\left(\mathbb{G}_{m}\right)=\left(E_{\mathbb{R}}^{n+p}(p) \oplus F^{p} E^{n+p} \oplus E^{n+p-1}\right)\left(\mathbb{G}_{m}\right)$ satisfies (2.9) (independently of $\alpha$, see the multiplication table in loc. cit.). The resulting spectrum $\mathrm{H}_{\mathrm{D}}$ sits in a distinguished triangle in $\mathbf{S H}\left(\mathbb{R}^{\text {an }}\right), \mathrm{H}_{\mathrm{D}} \rightarrow \mathrm{H}_{\mathrm{B}, \mathbb{R}}^{(4)} \rightarrow \mathrm{H}_{\mathrm{dR}}^{/ F \text {, an }}$ and thus, in $\mathbf{S H}(\mathbb{Q})$,

$$
c_{*} \operatorname{an}_{*} \mathrm{H}_{\mathrm{D}} \rightarrow c_{*} \mathrm{an}_{*}\left(\mathrm{H}_{\mathrm{B}, \mathbb{Q}}^{(1)} \otimes_{\mathbb{Q}} \mathbb{R}\right) \rightarrow \mathrm{H}_{\mathrm{dR}}^{/ F, \text { alg }} \otimes_{\mathbb{Q}} \mathbb{R} \rightarrow c_{*} \mathrm{an}_{*} \mathrm{H}_{\mathrm{D}}[1] .
$$

From now on, we write $\mathrm{H}_{\mathrm{D}}$ for $\operatorname{an}_{*} c_{*} \mathrm{H}_{\mathrm{D}} \in \mathbf{S H}(\mathbb{Q})$. This is the spectrum established in [HS11, Section 3], except for two inessential differences: instead of $\mathrm{D}(p)$, loc. cit. used other complexes that are homotopic (including the product structure, regardless of $\alpha$ ) to $\mathrm{D}(*)$. Secondly, the construction of loc. cit. builds a symmetric $\mathbb{P}^{1}$-spectrum, but again this is inessential at the level of the homotopy categories, since $-\wedge \mathbb{P}^{1}=-\wedge \mathbb{G}_{m} \wedge S_{s}^{1}$, where $S_{s}^{1}$ is the simplicial sphere. By HS11, Thm. $3.6]$,

$$
\operatorname{Hom}_{\mathbf{D M}_{\mathbf{b}}(\mathbb{Q})}\left(\mathrm{M}(X), \mathrm{H}_{\mathrm{D}}(p)[n]\right)=\operatorname{Hom}_{\mathbf{S H}(\mathbb{Q})}\left(\mathrm{M}(X), \mathrm{H}_{\mathrm{D}}(p)[n]\right)=\mathrm{H}_{\mathrm{D}}^{n}(X, p)
$$

for any $X \in \mathbf{S m} / \mathbb{Q}$. For any $M \in \mathbf{D M}_{\mathrm{b}, c}(\mathbb{Q}),(2.12)$ induces an isomorphism

$$
\begin{aligned}
\operatorname{det} \mathrm{H}_{\mathrm{D}}^{*}(M) & =\operatorname{det} c_{*} \mathrm{an}_{*} \mathrm{H}_{\mathrm{B}, \mathbb{R}}^{(4), *}(M) \otimes \operatorname{det}^{-1} c_{*} \mathrm{an}_{*} \mathrm{H}_{\mathrm{dR}}^{/ F, \text { an }}(M) \\
& =\left(\operatorname{det} c_{*} \mathrm{an}_{*} \mathrm{H}_{\mathrm{B}, \mathbb{Q}}^{(1), *}(M) \otimes \operatorname{det}^{-1} \mathrm{H}_{\mathrm{dR}}^{/ F, \mathrm{alg}, *}(M)\right) \otimes_{\mathbb{Q}} \mathbb{R} .
\end{aligned}
$$

Here $\operatorname{det} \mathrm{H}_{\mathrm{D}}^{*}(M):=\otimes_{n \in \mathbb{Z}} \operatorname{det}^{(-1)^{n}} \operatorname{Hom}\left(M, \mathrm{H}_{\mathrm{D}}[n]\right)$ etc. is well-defined since $M$ is compact. This is the promised extension of (2.1) to Deligne cohomology groups of general geometric motives.

Applied to the Betti realization, the exactness axiom (see Axiom 1.2(iii) means

$$
\operatorname{Hom}\left(M, \mathrm{H}_{\mathrm{B}, \mathbb{R}}\right)=\operatorname{Hom}\left({ }^{\mathrm{P}} \mathrm{H}^{0}(M), \mathrm{H}_{\mathrm{B}, \mathbb{R}}\right), \quad \text { for all } M \in \mathbf{D M}_{\mathrm{b}, c}(\mathbb{Q})
$$

and likewise for de Rham cohomology. This implies that for any smooth projective $X_{\eta} / \mathbb{Q}$

$$
\mathrm{H}_{\mathrm{D}}^{i}\left(\mathrm{~h}^{-b-1}\left(X_{\eta},-m\right)\right)= \begin{cases}\mathrm{H}_{\mathrm{D}}^{b+1}\left(X_{\eta}, m\right) & i=0 \text { and } b+1-2 m \geq 0 \\ \mathrm{H}_{\mathrm{D}}^{b+2}\left(X_{\eta}, m\right) & i=1 \text { and } b+1-2 m \leq-2 \\ 0 & \text { else. }\end{cases}
$$




\subsection{Arakelov motivic cohomology}

In order to formulate Conjecture 5.2 below, we need to recall some facts about Arakelov motivic cohomology.

Theorem 2.3. [HS11, Sch12a] In $\mathbf{D M}_{\mathrm{b}}(\mathbb{Z})$, there is a unique map ch : $\mathbf{1} \rightarrow \eta_{*} \mathrm{H}_{\mathrm{D}}$ representing the Chern class map from motivic cohomology to Deligne cohomology, i.e.

$$
\operatorname{Hom}_{\mathbf{D M}_{\mathbf{B}}(\mathbb{Z})}(\mathrm{M}(X), \mathbf{1}(p)[n]) \stackrel{\operatorname{ch}(p)[n]}{\longrightarrow} \operatorname{Hom}_{\mathbf{D M}_{\mathbf{B}}(\mathbb{Z})}\left(\mathrm{M}(X), \eta_{*} \mathrm{H}_{\mathrm{D}}(p)[n]\right)
$$

agrees with the Chern class $K_{2 p-n}(X)_{\mathbb{Q}}^{(p)} \rightarrow \mathrm{H}_{\mathrm{D}}^{n}\left(X_{\mathbb{Q}}, p\right)$ (also known as Beilinson regulator) for all regular projective schemes $X / \mathbb{Z}$. There is a certain, canonically defined object $\widehat{\mathbf{1}} \in \mathbf{D M}_{\mathrm{B}}(\mathbb{Z})$ called Arakelov motivic cohomology spectrum such that there is a distinguished triangle

$$
\widehat{\mathbf{1}} \stackrel{f}{\rightarrow} \mathbf{1} \stackrel{\mathrm{ch}}{\longrightarrow} \eta_{*} \mathrm{H}_{\mathrm{D}} \stackrel{\delta}{\longrightarrow} \widehat{\mathbf{1}}[1] .
$$

Moreover, given another object $\widehat{\mathbf{1}}^{\prime}$ in a similar triangle, there is a unique isomorphism $\widehat{\mathbf{1}} \rightarrow \widehat{\mathbf{1}}^{\prime}$ in $\mathbf{D M}_{\mathrm{b}}(\mathbb{Z})$ fitting in the obvious commutative diagram of distinguished triangles.

Definition 2.4. Given a motive $M \in \mathbf{D M}_{\mathrm{b}, c}(\mathbb{Z})$, its Arakelov motivic cohomology is defined as

$$
\widehat{\mathrm{H}}^{i}(M, m):=\operatorname{Hom}_{\mathbf{D M}_{\mathbf{B}}(\mathbb{Z})}(M, \widehat{\mathbf{1}}(m)[i]) .
$$

We write $\widehat{\mathrm{H}}^{i}(X, m):=\widehat{\mathrm{H}}^{i}(\mathrm{M}(X), m)$. We also consider the $\mathbb{R}$-linear variant of these groups, denoted $\widehat{H}_{\mathbb{R}}^{i}(X, m)$, obtained by replacing $\mathbf{1}$ by $\mathbf{1}_{\mathbb{R}}$ in (2.16). This amounts to tensoring the motivic cohomology groups with $\mathbb{R}$.

The triangle (2.16) induces long exact sequences

$$
\widehat{\mathrm{H}}_{\mathbb{R}}^{i}(M, m) \rightarrow \mathrm{H}^{i}(M, m)_{\mathbb{R}} \rightarrow \mathrm{H}_{\mathrm{D}}^{i}(M, m) \rightarrow \widehat{\mathrm{H}}_{\mathbb{R}}^{i+1}(M, m) .
$$

On the other hand, we have the notion of arithmetic $K$-theory. For a regular and projective scheme $X$ over $\mathbb{Z}$, such groups $\widehat{\mathrm{K}}_{n}^{T}(X)$ have been defined by Gillet and Soulé for $n=0$ and for higher $n$ by Takeda [GS90b, Section 6], [Tak05, p. 621]. These groups sit in an exact sequence

$$
K_{n+1}(X) \rightarrow \oplus_{p \in \mathbb{Z}} \mathrm{D}(p)^{2 p-n-1, G}(X) / \operatorname{im} d_{\mathrm{D}} \rightarrow \widehat{\mathrm{K}}_{n}^{T}(X) \rightarrow K_{n}(X) \rightarrow 0
$$

where $\mathrm{D}(p)^{G}$ is the complex defined in (2.11). Moreover, they come with a Chern class map ch $: \widehat{\mathrm{K}}_{n}^{T}(X) \rightarrow \oplus_{p \in \mathbb{Z}} \mathrm{D}(p)^{2 p-n, G}(X)$. The group $\widehat{\mathrm{K}}_{n}(X):=$ ker ch fits in a long exact sequence

$$
\ldots \rightarrow \oplus_{p \in \mathbb{Z}} \mathrm{H}_{\mathrm{D}}^{2 p-n-1}(X, p) \rightarrow \widehat{\mathrm{K}}_{n}(X) \rightarrow K_{n}(X) \rightarrow \oplus \mathrm{H}_{\mathrm{D}}^{2 p-n}(X, p) \rightarrow \ldots
$$

The group $\widehat{\mathrm{K}}_{0}^{T}(X)_{\mathbb{Q}}$ is also isomorphic, via the arithmetic Chern class to $\oplus_{p} \widehat{\mathrm{CH}}_{\mathrm{GS}}^{p}(X)_{\mathbb{Q}}$, where $\widehat{\mathrm{CH}}_{\mathrm{GS}}$ denotes the arithmetic Chow group of Gillet and Soulé [GS90a, 3.3.4]. It is generated by arithmetic cycles $\left(Z, g_{Z}\right)$, i.e., cycles $Z \subset X$ and Green currents, i.e., such that $\omega_{Z}:=\delta_{Z}-2 \partial \bar{\partial} g_{Z}$ is a differential form. Here $\delta_{Z}$ is the Dirac current. Under the arithmetic Chern class, the subgroup $\widehat{\mathrm{K}}_{0}(X)_{\mathbb{Q}} \subset \widehat{\mathrm{K}}_{0}^{T}(X)_{\mathbb{Q}}$ corresponds to the subgroup $\widehat{\mathrm{CH}}^{*}(X) \subset \widehat{\mathrm{CH}}_{\mathrm{GS}}^{*}(X)$ generated by arithmetic cycles $\left(Z, g_{Z}\right)$ such that $\omega_{Z}=0$ [GS90b, Thm. 7.3.4].

For a smooth scheme $X$ over $S \subset$ Spec $\mathbb{Z}$, the resulting decomposition of $\widehat{\mathrm{K}}_{0}(X)_{\mathbb{Q}}$ in Adams eigenspaces is extended to higher $\widehat{\mathrm{K}}$-theory [Sch12a, Cor. 6.2]: $\widehat{\mathrm{K}}_{n}(X)_{\mathbb{Q}}$ 
decomposes as a direct sum of Adams eigenspaces $\oplus \widehat{\mathrm{K}}_{n}(X)_{\mathbb{Q}}^{(p)}$, compatibly with (2.18). In fact, this statement is derived from a canonical isomorphism

$$
\widehat{\mathrm{H}}^{i}(X, m)=\widehat{\mathrm{K}}_{2 m-i}(X)_{\mathbb{Q}}^{(m)}\left(=\widehat{\mathrm{CH}}^{m}(X)_{\mathbb{Q}} \text { for } i=2 m\right) .
$$

Definition 2.5. Let $S \subset$ Spec $\mathbb{Z}$ be an open subscheme and let $M \in \mathbf{D M}_{\mathrm{B}}(S)$ be any motive. The natural pairing of motivic homology (see (1.2) ) and Arakelov motivic cohomology,

$$
\pi_{M}: \mathrm{H}_{-2}(M,-1)_{\mathbb{R}} \times \widehat{\mathrm{H}}_{\mathbb{R}}^{0}(M) \rightarrow \widehat{\mathrm{H}}_{\mathbb{R}}^{2}\left(\mathbf{1}_{S}, 1\right)
$$

given by the composition of morphisms in $\mathbf{D M}_{\mathrm{b}}(S)$ is called Arakelov intersection pairing.

Remark 2.6. (i) For $M \in \mathbf{D M}_{\mathrm{b}, c}(S)$, we often tacitly identify $\mathrm{H}_{-2}(M,-1) \cong$ $\mathrm{H}^{2}\left(M^{\vee}, 1\right)$, cf. (1.4).

(ii) The Arakelov intersection pairing is functorial in $M$ in an obvious sense.

(iii) Let $M \in \mathbf{D M}_{\mathrm{b}, c}(S)$. Consider

$$
\begin{array}{cccccc}
\widehat{\mathrm{H}}_{\mathbb{R}}^{0}(M) & \times & \mathrm{H}_{\mathbb{R}}^{2}\left(M^{\vee}, 1\right) & \longrightarrow & \widehat{\mathrm{H}}^{2}(\mathbf{1}, 1) \\
\downarrow & & \uparrow & & \downarrow= \\
\mathrm{H}_{\mathbb{R}}^{0}(M) & \times & \widehat{\mathrm{H}}_{\mathbb{R}}^{2}\left(M^{\vee}, 1\right) & \longrightarrow & \widehat{\mathrm{H}}^{2}(\mathbf{1}, 1) \\
\downarrow & & \uparrow & & \uparrow \cong \\
\mathrm{H}_{\mathrm{D}}^{0}(M) & \times & \mathrm{H}_{\mathrm{D}}^{1}\left(M^{\vee}, 1\right) & \longrightarrow & \mathrm{H}_{\mathrm{D}}^{1}(\mathbf{1}, 1),
\end{array}
$$

where in the first row $\left(a: M \rightarrow \widehat{\mathbf{1}}, b: M^{\vee} \rightarrow \mathbf{1}\{-1\}\right)$ is mapped to $\mu \circ(a \otimes b) \circ$ coev, where the coevaluation $\mathbf{1} \rightarrow M \otimes M^{\vee}$ is obtained from (1.4), $\mu: \mathbf{1} \otimes \widehat{\mathbf{1}} \rightarrow \widehat{\mathbf{1}}$ is the $\mathbf{1}$-module structure map for $\widehat{\mathbf{1}}$. This is just another way to write $\pi_{M}$. Likewise, the second row pairing is $\pi_{M^{\vee}\{-1\}}$. The pairing in the third row is defined similarly using the product of the ring spectrum $\mu_{\mathrm{D}}: \mathrm{H}_{\mathrm{D}} \otimes \mathrm{H}_{\mathrm{D}} \rightarrow \mathrm{H}_{\mathrm{D}}$ instead. This diagram is commutative. This follows from the commutativity of the following diagram, which in turn is a rephrasing of the fact that (2.16) is a distinguished triangle of $\mathbf{1}$-modules.

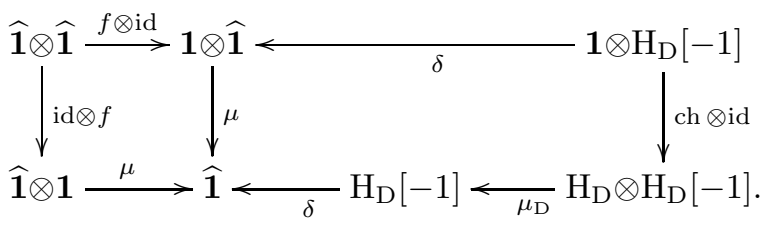

(iv) The pairing $\mathrm{H}_{\mathrm{D}}^{0}(M) \times \mathrm{H}_{\mathrm{D}}^{1}\left(M^{\vee}, 1\right) \rightarrow \mathbb{R}$ is a perfect pairing for any $M$. It suffices to see this for $M=\mathrm{M}(X)(p)[n]$ for $X / \mathbb{Z}$ regular and projective, in which case it follows from the identification of Deligne cohomology with weak Hodge cohomology (Lemma 2.2) and the duality of weak Hodge cohomology, (2.5). This plays an important role in the compatibility of our $L$-values conjecture with respect to the functional equation, see Theorem [5.5)(ii).

Example 2.7. Consider a motive $M=i_{*} N$, where $i:$ Spec $\mathbb{F}_{p} \rightarrow$ Spec $\mathbb{Z}$ and $N \in \mathbf{D M}_{\mathrm{b}, c}\left(\mathbb{F}_{p}\right)$ (for example $M=\mathrm{M}\left(\mathbb{F}_{p}\right)=i_{*} i^{*} \mathbf{1}\{-1\}$ ). The forgetful map $f: \widehat{\mathrm{H}}_{\mathbb{R}}^{0}(M) \rightarrow \mathrm{H}^{0}(M)_{\mathbb{R}}=\mathrm{H}^{-2}(N,-1)_{\mathbb{R}}$ is an isomorphism and the pairing $\pi_{M}$ coincides with the natural pairing $\mathrm{H}_{-2}(N,-1)_{\mathbb{R}} \times \mathrm{H}^{-2}(N,-1)_{\mathbb{R}} \rightarrow \mathrm{H}^{0}\left(\mathbf{1}_{\mathbb{F}_{p}}, 0\right)_{\mathbb{R}}=\mathbb{R}$ followed by the pushforward $i_{*}: \widehat{\mathrm{H}}^{0}\left(\mathbf{1}_{\mathbb{F}_{p}}, 0\right)_{\mathbb{R}} \rightarrow \widehat{\mathrm{H}}_{\mathbb{R}}^{2}\left(\mathbf{1}_{\mathbb{Z}}, 1\right)$, which is $\log p: \mathbb{R} \rightarrow \mathbb{R}$ [Sch12a, Theorem 6.4.(i)]. 
Example 2.8. Let $X$ be a regular projective scheme over $S \subset$ Spec $\mathbb{Z}$ of constant dimension $d$. We pick some open $j: U \subset S$ such that $X_{U}$ is smooth over $U$. Let $M:=\mathrm{M}(X)\{m-d\}[i] \in \mathbf{D M}_{\mathrm{b}, c}(S)$. Then $\mathrm{H}_{-2}(M,-1)=K_{i}(X)_{\mathbb{Q}}^{(m)}$ by absolute purity. Let $M_{U}:=j^{*} M \in \mathbf{D M}_{\mathrm{b}, c}(U)$. Consider

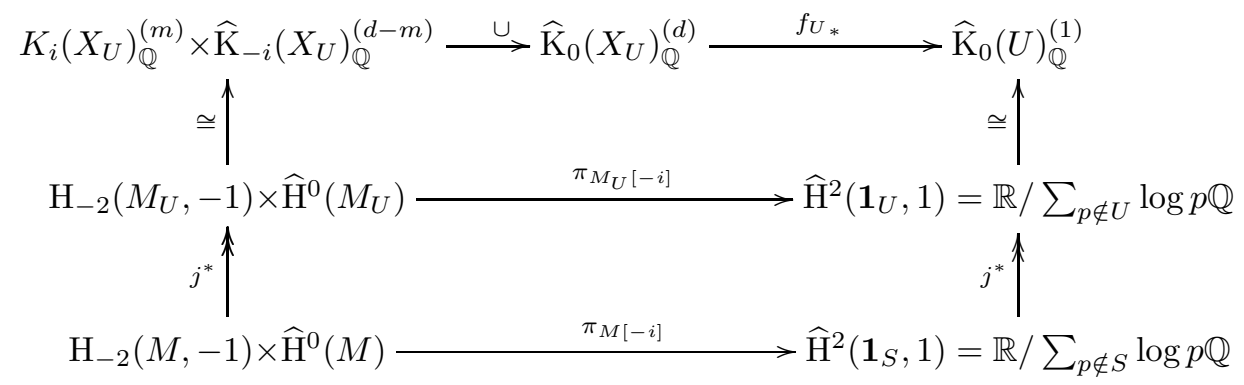

In the first row, the pushforward $f_{U_{*}}$ is not the pushforward on arithmetic $K$ theory, but the one on arithmetic Chow groups using the arithmetic Chern class isomorphism (2.19). The top square is commutative by [Sch12a, Thm. 7.4.]. The bottom square is commutative by definition. See also Remark 5.7

\section{$3 \quad L$-functions of motives over number rings}

Let $F$ be a number field and $\mathcal{O}_{F}$ its ring of integers. For every finite prime $\mathfrak{p}$ of $\mathcal{O}_{F}$ we fix a rational prime $\ell$ that does not lie under $\mathfrak{p}$. Moreover, fix for every $\ell$ an embedding $\sigma_{\ell}: \mathbb{Q}_{\ell} \rightarrow \mathbb{C}$. All subsequent definitions of $L$-functions are taken with respect to these choices.

Definition 3.1. The $L$-series of a mixed motive $M_{\eta}$ over $F$ is defined by

$$
L_{F}\left(M_{\eta}, s\right):=\prod_{\mathfrak{p}<\infty} \operatorname{det}\left(\operatorname{Id}-\operatorname{Fr}^{-1} \cdot N(\mathfrak{p})^{-s} \mid\left(M_{\eta_{\ell}} \otimes_{\mathbb{Q}_{\ell}, \sigma_{\ell}} \mathbb{C}\right)^{I_{\mathfrak{p}}}\right)^{-1} .
$$

The $L$-series of a geometric motive $M$ over $\mathcal{O}_{F}$ is given by

$$
L_{\operatorname{Spec} \mathcal{O}_{F}}(M, s):=L(M, s):=\prod_{\mathfrak{p}<\infty} \operatorname{det}\left(\operatorname{Id}-\operatorname{Fr}^{-1} \cdot N(\mathfrak{p})^{-s} \mid\left(i_{\mathfrak{p}}^{!} M\right)_{\ell} \otimes_{\mathbb{Q}_{\ell}, \sigma_{\ell}} \mathbb{C}\right)^{-1} .
$$

The first definition is classical, the second is a natural adaptation to motives over $\mathcal{O}_{F}$. The products run over all finite primes of $\mathcal{O}_{F}, \mathrm{Fr}$ is the arithmetic Frobenius map (given on residue fields by $\left.a \mapsto a^{N(\mathfrak{p})}\right), N(\mathfrak{p})$ is the norm of $\mathfrak{p}, i_{\mathfrak{p}}$ denotes the immersion of the corresponding closed point and $-_{\ell}$ denotes the $\ell$-adic realization functor, see (1.6). The determinants are understood in the sense of Section 1.1. The superscript $I_{\mathfrak{p}}$ denotes the invariants under the action of the inertia group.

Remark 3.2. By [Sch12b, Axiom 4.5.], the $\ell$-adic realization $M_{\eta_{\ell}}$ is in fact an $\ell$-adic sheaf. For example, $\left(\mathrm{h}^{-b-1}\left(X_{\eta},-m\right)\right)_{\ell}=\mathrm{H}^{b+1}\left(X_{\eta}, \mathbb{Q}_{\ell}(m)\right)$ for some scheme $X_{\eta}$ over $F$.

The independence of the choices of $\ell$ and the embeddings $\sigma_{\ell}$ is discussed around Lemma 3.10. See also Theorem 4.3 .

The $L$-series for motives over $\mathcal{O}_{F}$ is multiplicative, i.e., given a triangle $M \rightarrow$ $M^{\prime} \rightarrow M^{\prime \prime}$ in $\mathbf{D M}_{\mathrm{b}, c}\left(\mathcal{O}_{F}\right)$, one gets

$$
L\left(M^{\prime}, s\right)=L(M, s) \cdot L\left(M^{\prime \prime}, s\right) .
$$

A similar property does not hold for $L$-functions of motives over $F$ [Sch91]. See also FPR94, 1.3.3]. 
By definition and the calculation of $\ell$-adic cohomology of $\mathbb{P}_{\mathbb{F}_{\mathfrak{p}}}^{1}$, one has

$$
L(M(-m), s)=L(M, m+s), \quad m \in \mathbb{Z} .
$$

For an open subscheme $j:$ Spec $\mathcal{O}_{F} \backslash Z \rightarrow$ Spec $\mathcal{O}_{F}$ with complement $i: Z \rightarrow$ Spec $\mathcal{O}_{F}$, the $L$-function of $j_{*} j^{*} M$ is the one of $M$, but the Euler factors for the points in $Z$ are omitted. This follows from $i ! j_{*}=0$.

The following lemma is well-known, see [Del73, Prop. 3.8.(ii)] or Neu92, VII.10.4.(iv)] for similar statements. It permits to replace any number ring $\mathcal{O}_{F}$ by $\mathbb{Z}$ and to study $L$-values of motives over $\mathbb{Z}$, only.

Lemma 3.3. The L-series is an absolute invariant of a motive, i.e., for any geometric motive $M$ over Spec $\mathcal{O}_{F}$ we have $L_{\operatorname{Spec}} \mathcal{O}_{F}(M, s)=L_{\text {Spec } \mathbb{Z}}\left(f_{*} M, s\right)$, where $f:$ Spec $\mathcal{O}_{F} \rightarrow$ Spec $\mathbb{Z}$ denotes the structural map.

We now relate $L$-series of motives over $\mathbb{Q}$ to ones over $\mathbb{Z}$. Recall the notion of smooth motives from Definition 1.1. The following lemma is proven in Sch12b, Section 5.5] as a corollary of the exactness axiom for $\ell$-realization functors (see around (1.7)).

Lemma 3.4. Let $M$ be a mixed smooth motive over $U$, where $j: U \rightarrow$ Spec $\mathbb{Z}[1 / \ell]$ is an open subscheme. Let $i$ be the complementary closed immersion to $j$ and let $\eta^{\prime}$ and $\eta$ be the generic point of $U$ and Spec $\mathbb{Z}[1 / \ell]$, respectively. Then $\left(i ! j_{! *} M\right)_{\ell}=$ $i^{*}\left(\mathrm{R}^{0} \eta_{*} \eta^{\prime *} M_{\ell}[1]\right)[-1]$.

The following proposition relates $L$-series of motives over $\mathbb{Q}$ and $\mathbb{Z}$. Our main example is $M_{\eta}=\mathrm{h}^{-b-1}\left(X_{\eta},-m\right)$ and $M=\mathrm{h}^{-b}(X,-m)$ where $X / \mathbb{Z}$ is some projective scheme whose generic fiber $X_{\eta} / \mathbb{Q}$ is smooth.

Proposition 3.5. Let $M_{\eta} \in \mathbf{M M}(\mathbb{Q})$. Pick some $M \in \mathbf{M M}(\mathbb{Z})$ with $M_{\eta}=$ $\eta^{*}[-1] M$. Then

$$
L_{\mathbb{Q}}\left(M_{\eta}, s\right)^{-1}=L_{\mathbb{Z}}\left(\eta_{! *} \eta^{*} M, s\right) .
$$

Proof: For sufficiently small $j: U \rightarrow \operatorname{Spec} \mathbb{Z}$, the right hand side is equal to

$$
\begin{aligned}
L_{\mathbb{Z}}\left(j_{! *} j^{*} M, s\right) & \stackrel{\text { 3.4. }}{=}\left(\prod_{p} \operatorname{det}\left(\mathrm{Id}-\mathrm{Fr}^{-1} p^{-s} \mid i_{p}^{*}\left(\mathrm{R}^{0} \eta_{*} \eta^{*} M_{\ell}[1]\right)[-1]\right)\right)^{-1} \\
& =\prod_{p} \operatorname{det}\left(\operatorname{Id}-\mathrm{Fr}^{-1} p^{-s} \mid i_{p}^{*} \mathrm{R}^{0} \eta_{*} M_{\eta_{\ell}}\right) \\
& =\prod_{p} \operatorname{det}\left(\operatorname{Id}-\mathrm{Fr}^{-1} p^{-s} \mid\left(M_{\eta_{\ell}}\right)^{I_{p}}\right)=L_{\mathbb{Q}}\left(M_{\eta}, s\right)^{-1}
\end{aligned}
$$

\subsection{Hasse-Weil $\zeta$-functions - Motives with compact support}

Definition 3.6. (see e.g. Ser65]) The Hasse-Weil zeta function of a scheme $X / \mathbb{Z}$ (always separated and of finite type) is defined as $\zeta(X, s):=\prod_{x}\left(1-N(x)^{-s}\right)^{-1}$. The product is over all closed points $x$ of $X$, and $N(x)$ denotes the cardinality of the (finite) residue field of $x$.

Recall from (1.1) the motive with compact support $\mathrm{M}_{\mathrm{c}}(X)$ of some scheme $X$.

Proposition 3.7. For any scheme $X / \mathbb{Z}$, we have

$$
\zeta(X, s)=L\left(\mathrm{M}_{\mathrm{c}}(X), s\right) .
$$


Proof: Writing $i_{p}:$ Spec $\mathbb{F}_{p} \rightarrow$ Spec $\mathbb{Z}$ and $X_{p}:=X \times \mathbb{F}_{p}$, base-change implies $i_{p *} i_{p}^{!} \mathrm{M}_{\mathrm{c}}(X)=\mathrm{M}_{\mathrm{c}}\left(X_{p}\right)$. (At the right hand side, $X_{p}$ is seen as a $\mathbb{Z}$-scheme.) Therefore, $L\left(\mathrm{M}_{\mathrm{c}}(X), s\right)=\prod_{p} L\left(\mathrm{M}_{\mathrm{c}}\left(X_{p}\right), s\right)$. A similar decomposition for the $\zeta$-function allows us to assume that $X$ is an $\mathbb{F}_{p}$-scheme. The $\ell$-adic realization functor satisfies $\left(f_{*} f^{!} \mathbf{1}\right)_{\ell}=f_{!} f^{*} \mathbb{Q}_{\ell}$. Grothendieck's trace formula (see e.g. Mil80, Sections VI.12, 13]) says

$$
\begin{aligned}
\zeta(X, s) & =\prod_{i=0}^{2 \operatorname{dim} X}\left(\operatorname{det}\left(\operatorname{Id}-\mathrm{Fr}^{-1} \cdot p^{-s} \mid \mathrm{H}_{c}^{i}\left(X \times_{\mathbb{F}_{p}} \overline{\mathbb{F}}_{p}, \mathbb{Q}_{\ell}\right)\right)\right)^{(-1)^{i+1}} \\
& =\operatorname{det}\left(\operatorname{Id}-\mathrm{Fr}^{-1} \cdot p^{-s} \mid f_{!} f^{*} \mathbb{Q}_{\ell}\right)^{-1}
\end{aligned}
$$

where $\mathrm{H}_{c}^{i}\left(X \times \overline{\mathbb{F}}_{p}, \mathbb{Q}_{\ell}\right)=\mathrm{H}^{i}\left(f_{!} f^{*} \mathbb{Q}_{\ell}\right)$ denotes $\ell$-adic cohomology with compact support.

The $L$-series of a motive over $\mathbb{Q}$ is conjectured to be independent of the choice of $\ell$ and $\sigma_{\ell}$ in every factor (assuming $p \neq \ell$ ). This is known for the individual Euler factors at $p$ if the motive is $\mathrm{h}^{i}\left(X_{\eta}, n\right)$, where $X_{\eta}$ is a variety with good reduction at $p$, by Deligne's work on the Weil conjectures [Del74, Th. 1.6]. From Proposition 3.7 we now immediately obtain another statement concerning independence of $\ell$.

Definition 3.8. The smallest triangulated subcategory of $\mathbf{D M}_{\mathrm{b}, c}(\mathbb{Z})$ containing the motives $\mathrm{M}(X)(n)(n \in \mathbb{Z})$ of all regular schemes $X$ which are projective and flat over $\mathbb{Z}$, and the image of $i_{*}: \mathbf{D M}_{\mathrm{b}, c}\left(\mathbb{F}_{p}\right) \rightarrow \mathbf{D M}_{\mathrm{b}, c}(\mathbb{Z})$ for all primes $p$, is called $\mathbf{D M}_{\mathrm{B}, \operatorname{tr}}(\mathbb{Z})$ and called category of accessible motives. Its triangulated subcategory generated by $\mathrm{M}(X)(n)$ where $X$ is regular and projective, but not necessarily flat over $\mathbb{Z}$ (such as a smooth projective $X / \mathbb{F}_{p}$ ) is called the category of easily accessible motives.

Remark 3.9. (i) By de Jong's resolution of singularities using alterations, the thick closure (i.e., closure under direct summands and triangles) of the category of easily accessible motives contains the motives $\mathrm{M}(X)(n)$ of all $X$ schemes (of finite type) over $\mathbb{Z}$. Therefore, this thick closure is the entire category $\mathbf{D M}_{\mathrm{b}, c}(\mathbb{Z})$ of geometric motives.

(ii) By the proof of $\left[\underline{S c h 12 b}\right.$, Prop. 5.6], $\mathbf{D M}_{\mathrm{b}, \mathrm{tr}}(\mathbb{Z})$ is contained in the triangulated category generated by $i_{*} \mathbf{D M}_{\mathrm{b}, c}\left(\mathbb{F}_{p}\right)$ and motives of the form $E:=$ $\eta ! * \eta^{*} \mathrm{~h}^{-b}(X,-m)$, where $X / \mathbb{Z}$ is regular, flat and projective.

The following lemma shows that the question of independence of $L$-functions of $\ell$ is solely about the behavior of $L$-functions under direct summands.

Lemma 3.10. For any easily accessible motive $M$ over $\mathbb{Z}$, the $L$-series $L(M, s)$ does not depend on the choices of $\ell$ (provided $p \nmid \ell$ ) and $\sigma_{\ell}$.

Proof: Using (3.1), we may assume $M=\mathrm{M}(X)=\mathrm{M}_{\mathrm{c}}(X)$ for some $X$ which is projective over $\mathbb{Z}$ (and regular). Then the claim immediately follows from Proposition 3.7 .

\subsection{Meromorphic continuation and functional equation}

Properties of $L$-series for motives over $\mathbb{Q}$ tend to generalize to ones over $\mathbb{Z}$, given that the property in question is known for motives over $\mathbb{F}_{p}$. We illustrate this by the absolute convergence, meromorphic continuation, and the functional equation. Recall from [Del79, 5.2.] or [Sch88, p. 4] the definition of the archimedean Euler factor $L_{\infty}(V, s)$ for a mixed Hodge structure $V$. Essentially, $L_{\infty}(V, s)$ is a product 
of $\Gamma$-functions. The pole order at $s=0$ is given by ([Ber86, Lemma 7.1.] or [FPR94, III.1.2.5 + III.1.2.3]):

$$
\operatorname{ord}_{s=0} L_{\infty}(V, s)=-\operatorname{dim}_{\mathbb{R}} \mathrm{H}_{\mathrm{w}}^{1}\left(V^{\vee}(1)\right) .
$$

For $V_{*} \in \mathbf{D}_{\mathrm{H}}^{\mathrm{b}}$, we put $L_{\infty}\left(V_{*}, s\right):=\prod_{i \in \mathbb{Z}} L_{\infty}\left(\mathrm{H}^{i}\left(V_{*}\right), s\right)^{(-1)^{i}}$. Here $\mathrm{H}^{i}\left(V_{*}\right)$ denotes the $i$-th cohomology Hodge structure of the complex $V_{*}$.

Definition 3.11. Let $M$ be a geometric motive over $\mathbb{Z}$ or a mixed motive over $\mathbb{Q}$. The function

$$
L_{\infty}(M, s):=L_{\infty}\left(\mathrm{R} \Gamma_{\mathrm{H}}(M), s\right)
$$

is called the archimedean factor of the $L$-function of $M$. Here $\mathrm{R} \Gamma_{\mathrm{H}}$ is the Hodge realization functor (2.7). The completed $L$-function of $M$ is defined as

$$
\Lambda(M, s):=L(M, s) L_{\infty}(M, s) .
$$

Much the same way as $L$-functions of motives over $\mathbb{Q}$, archimedean factors are not multiplicative with respect to short exact sequences of Hodge structures. (See [FPR94, 1.1.9, 1.2.5] for a necessary and sufficient criterion for multiplicativity.)

The following is a long-standing conjecture concerning $L$-functions Del73, [Del79, 5.2, 5.3] or [FPR94, p. 610, 699]:

Conjecture 3.12. Let $M_{\eta}$ be a mixed motive over $\mathbb{Q}$. The $L$-series $L_{\mathbb{Q}}\left(M_{\eta}, s\right)$ converges absolutely for $\Re(s) \gg 0$ and has a meromorphic continuation to the complex plane. There is a functional equation relating the $\Lambda$-functions of $M_{\eta}$ and $M_{\eta}^{\vee}(-1)$ :

$$
\Lambda\left(M_{\eta}, s\right)=\epsilon(M, s) \Lambda\left(M_{\eta}^{\vee}(-1),-s\right),
$$

where $\epsilon(M, s)$ is of the form $a b^{s}$, with nonzero constants $a$ and $b$ depending on $M$.

Lemma 3.13. Conjecture 3.12 implies the following: for any accessible motive $M$ over $\mathbb{Z}$ (Definition [3.8), the L-series $L(M, s)$ converges absolutely for $\Re(s) \gg 0$, has a meromorphic continuation to the complex plane, and there is a functional equation $\Lambda(M, s)=\epsilon(M, s) \Lambda\left(M^{\vee}(-1),-s\right)$, where $\epsilon(M, s)$ is of the form ab $b^{s}$, with nonzero constants $a$ and $b$ depending on $M$.

Proof: The claim is triangulated, since the assignments $M \mapsto L(M, s)$, and $M \mapsto L_{\infty}(M, s) / L_{\infty}\left(M^{\vee}(-1),-s\right)$ are multiplicative for $M \in \mathbf{D M}_{\mathrm{b}, c}(\mathbb{Z})$, the latter up to sign [FPR94, Prop. III.1.2.8]. By Remark 3.9(1ii), it is enough to show the claim for $M=i_{*} N, N \in \mathbf{D M}_{\mathrm{b}, c}\left(\mathbb{F}_{p}\right)$ and $M=E:=\eta_{! *} \eta^{*} \mathrm{~h}^{-b}(X,-m)$, where $X / \mathbb{Z}$ is regular, flat and projective. For $M=E$, we have $L(M, s)=L_{\mathbb{Q}}\left(\mathrm{h}^{-b-1}\left(X_{\eta}\right), s\right)^{-1}$. This and the formula (5.2) for $M^{\vee}(-1)$ in this case shows that the conjectural (see 3.12) properties of $L_{\mathbb{Q}}\left(\mathrm{h}^{-b-1}\left(X_{\eta}\right), s\right)$ implies the same properties for $L(M, s)$. The $L$-series of $M=i_{*} N$ is a rational function in $p^{-s}$ (a priori with complex coefficients), which immediately yields the convergence for $\Re(s) \gg 0$ and the meromorphicity. Noting that $\left(i_{*} N\right)^{\vee}\{-1\}=i_{*}\left(N^{\vee}\right)$, the functional equation also holds unconditionally, as is well-known.

Remark 3.14. Under Conjecture 1.5 the constant $a$ above is rational for $M=i_{*} N$, where $i:$ Spec $\mathbb{F}_{p} \rightarrow$ Spec $\mathbb{Z}$. To see this, we may assume by triangulatedness that $N$ is a pure motive with respect to numerical or homological equivalence, so that its $L$-function is a rational function in $p^{-s}$ with rational coefficients (see the reference in the proof of Theorem [5.20). 


\section{Is the Arakelov intersection pairing perfect?}

Conjecture 4.1. For any geometric motive $M$ over $\mathbb{Z}, M \in \mathbf{D M}_{\mathrm{b}, c}(\mathbb{Z})$ (see Section 1.2 for the notation), the Arakelov intersection pairing between motivic homology and Arakelov motivic cohomology (Definition 2.5)

$$
\pi_{M}: \mathrm{H}_{-2}(M,-1)_{\mathbb{R}} \times \widehat{\mathrm{H}}_{\mathbb{R}}^{0}(M) \rightarrow \mathbb{R}
$$

is a perfect pairing of finite-dimensional $\mathbb{R}$-vector spaces.

Remark 4.2. (i) The shape of (4.1) is similar to the situation of étale constructible sheaves over Spec $\mathbb{Z}$ : thinking of $M \in \mathbf{D M}_{\mathrm{B}, c}(\mathbb{Z})$ as being analogous to a complex of constructible sheaves $\mathcal{F}$ over $\mathbb{Z}$, the groups $\mathrm{H}_{\mathrm{D}}^{*}(M)$ correspond (in spirit) to the Tate cohomology groups $\mathrm{H}_{\text {Tate }}^{*}\left(\mathbb{R},\left.\mathcal{F}\right|_{\mathbb{R}}\right)$ at the archimedean place. Given that, $\widehat{\mathrm{H}}^{i}(M)$ parallels $\mathrm{H}_{\mathrm{c}}^{i}(\mathcal{F}):=\mathrm{H}^{i} \mathrm{R} \Gamma_{\mathrm{c}}(\mathbb{Z}, \mathcal{F})$, that is to say, cohomology with compact support, which is defined via $\mathrm{R} \Gamma_{\mathrm{c}}:=$ cone $[-1]\left(\mathrm{R} \Gamma(\mathbb{Z}, \mathcal{F}) \rightarrow \mathrm{R} \Gamma_{\text {Tate }}\left(\mathbb{R},\left.\mathcal{F}\right|_{\mathbb{R}}\right)\right)$, much the same way as (2.16), (2.17). Finally, the Arakelov intersection pairing corresponds to the perfect pairing of Artin-Verdier duality, see e.g. [Mil06, Ch. II.3]

$$
\mathrm{H}_{\mathrm{c}}^{i}(\mathbb{Z}, \mathcal{F}) \times \operatorname{Ext}_{\mathbb{Z}}^{3-i}\left(\mathcal{F}, \mathbb{G}_{m}\right) \rightarrow \mathrm{H}_{\mathrm{c}}^{3}\left(\mathbb{Z}, \mathbb{G}_{m}\right) .
$$

A higher-dimensional extension was conjectured by Milne [Mil06, Conjecture II.7.17] and proven by Geisser Gei10.

(ii) For any fixed $M \in \mathbf{D M}_{\mathrm{b}, c}(\mathbb{Z})$, Conjecture 4.1 for all $M[k](k \in \mathbb{Z})$ is equivalent to the one for $M^{\vee}\{-1\}[k]$. This follows from Remark 2.6(iii), (iv) and the five lemma.

(iii) Gillet and Soulé conjecture that the intersection product

$$
\widehat{\mathrm{CH}}_{\mathrm{GS}}^{m}(X)_{\mathbb{R}} \times \widehat{\mathrm{CH}}_{\mathrm{GS}}^{d-m}(X)_{\mathbb{R}} \rightarrow \mathbb{R}
$$

is non-degenerate for any regular scheme $X$ that is projective and flat over $\mathbb{Z}$ of constant dimension $d$ [GS94, Conjecture 1]. By Example 2.8 at least for $X$ smooth, this pairing is compatible with the Arakelov intersection pairing $\pi_{\mathrm{M}(X)\{m-d\}}$, i.e., there is a commutative diagram of pairings,

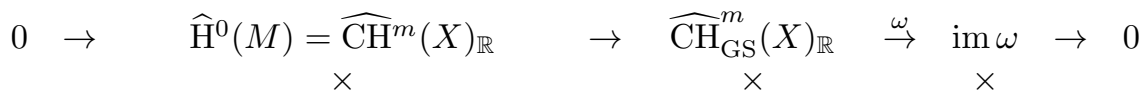

$$
\begin{aligned}
& 0 \leftarrow \mathrm{H}_{-2}(M,-1)=\mathrm{CH}^{d-m}(X)_{\mathbb{R}} \leftarrow{\widehat{\mathrm{CH}_{\mathrm{GS}}}}^{d-m}(X)_{\mathbb{R}} \leftarrow \operatorname{im} a \leftarrow 0 \\
& \begin{array}{lll}
\downarrow & \downarrow & \downarrow \\
\mathbb{R} & \mathbb{R} & \mathbb{R}
\end{array}
\end{aligned}
$$

where $\omega: \widehat{\mathrm{CH}}_{\mathrm{GS}}^{m}(X) \rightarrow A^{m, m}(X)$ and $a: A^{d-m-1, d-m-1}(X) /(\operatorname{im} \partial+\operatorname{im} \bar{\partial}) \rightarrow$ $\widehat{\mathrm{CH}}_{\mathrm{GS}}^{d-m}(X)$ are defined in [GS90a, Section 3.3.4]. I don't know whether the pairing on the right is a non-degenerate pairing, so the relation of Gillet-Soulé's conjecture and 5.2 is unclear. Note that $\operatorname{im} \omega$ and $\operatorname{im} a$ are infinite-dimensional $\mathbb{R}$-vector spaces.

Next, we show that Conjecture 4.1 recovers all the axioms on mixed motives over $\mathbb{F}_{p}$ we were willing to assume. Previously, it was known that Tate's conjecture about the pole order of $\zeta$-functions over finite fields and Conjecture 1.5 together imply the Beilinson-Parshin conjecture Gei98, Thm. 1.2.], and that the Beilinson-Parshin conjecture is equivalent to Bondarko's weight functor $\mathbf{D M}_{\mathrm{gm}}^{\mathrm{eff}}\left(\mathbb{F}_{p}\right) \rightarrow \mathbf{K}^{\mathrm{b}}\left(\mathrm{M}_{\text {rat }}^{\mathrm{eff}}\right)$ between the triangulated category of effective motives with the bounded homotopy category of effective Chow motives (with rational coefficients) being an equivalence of categories Bon09, Section 8.3.2]. 
Theorem 4.3. Conjecture 4.1 for motives of the form $M=i_{*} N$ ( $N$ any geometric motive over $\mathbb{F}_{p}, i:$ Spec $\mathbb{F}_{p} \rightarrow$ Spec $\mathbb{Z}$ ) is equivalent to the conjunction of Conjecture 1.5 and the Beilinson-Parshin conjecture stating

$$
K_{r}(X)_{\mathbb{Q}}=0
$$

for any smooth projective variety $X$ over $\mathbb{F}_{p}$ and all $r>0$.

Under the axioms concerning the existence and cohomological dimension of mixed motives over $\mathbb{F}_{p}$ and the weight formalism (see Axiom [1.2), Conjecture 4.1] for all motives $i_{*} N$ is equivalent to Conjecture 1.5.

Proof: Using the axioms about mixed motives, we first show that Conjecture 1.5 implies the perfectness. By construction, cf. (2.17), $\widehat{\mathrm{H}}_{\mathbb{R}}^{*}\left(i_{*} N\right)=\mathrm{H}^{*}(N)_{\mathbb{R}}$. By Sch12b Axiom 4.1.], the cohomological dimension of $\mathbf{D M}_{\mathrm{E}, c}\left(\mathbb{F}_{p}\right)$ is zero, so that $\mathrm{H}^{j}(N)=\mathrm{H}^{0}\left({ }^{\mathrm{p}} \mathrm{H}^{j} N\right)$ and similarly for $N^{\vee}$. By the same axiom, only finitely many $j$ yield a non-zero term. Therefore, we may replace $N$ by ${ }^{\mathrm{p}} \mathrm{H}^{j} N$ and assume that $N$ is a mixed motive. Using the weight filtration we reduce to the case where $N$ is a pure motive. Under Conjecture 1.5. all adequate equivalence relations agree, so we may regard $N$ as a Chow motive or as a pure motive with respect to numerical equivalence. By the semi-simplicity of pure numerical motives there is a decomposition $N=\mathbf{1}^{r} \oplus R$, where $R$ satisfies $\mathrm{H}_{\mathbf{D M}_{\mathrm{B}, c}^{0}\left(\mathbb{F}_{p}\right)}\left(R^{\vee}\right)=\mathrm{H}_{\mathbf{D M}_{\mathrm{B}, c}\left(\mathbb{F}_{p}\right)}(R)=0$. By functoriality of the pairing we get a commutative diagram

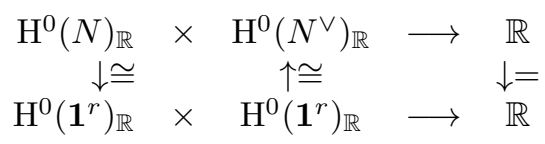

The lower line is a perfect pairing, since the one for $\mathbf{1}_{\mathbb{F}_{p}}$ is by Example 2.7

We now show the second statement. Let $X$ be a smooth equidimensional projective variety over $\mathbb{F}_{q}$. We regard it as a $\mathbb{Z}$-scheme. By Example 2.7 the Arakelov intersection pairing

$$
\widehat{\mathrm{K}}_{2 m-k}(X)^{(m)} \times K_{k-2 m}(X)_{\mathbb{Q}}^{(\operatorname{dim} X-m)}=K_{2 m-k}(X)_{\mathbb{R}}^{(m)} \times K_{k-2 m}(X)_{\mathbb{R}}^{(\operatorname{dim} X-m)} \rightarrow \mathbb{R}
$$

is the usual multiplication on Adams eigenspaces in $K$-theory, followed by the multiplication with $\log p$ (which is irrelevant for the question of the perfectness). For $2 m-k>0$ the second factors vanishes, hence the perfectness is equivalent to (4.3). For $2 m=k$ is perfectness is equivalent, by definition, to the agreement of numerical and rational equivalence (up to torsion). This shows one implication of the second statement. By resolution of singularities, the category $\mathbf{D} \mathbf{M}_{\mathrm{b}, c}\left(\mathbb{F}_{p}\right)$ is generated as a thick category by motives $\mathrm{M}(X)(m)$ as above. Since the perfectness only has to be checked on such generators, we are done with the converse implication as well.

The following corollary was pointed out to me by Bruno Kahn.

Corollary 4.4. The perfectness of $\pi_{M}$ for all motives $M=i_{*} N$ implies a canonical equivalence $\mathbf{D M}_{\mathrm{b}, c}\left(\mathbb{F}_{p}\right)=\mathbf{D}^{\mathrm{b}}\left(\mathbf{M}_{\text {rat }}\left(\mathbb{F}_{p}\right)\right)$, which in turn implies among other things the independence of $L$-functions of $\ell$.

Proof: That description of $\mathbf{D M}_{\mathrm{b}, c}\left(\mathbb{F}_{p}\right)$ is a consequence of $\sim_{\text {num }}=\sim_{\text {rat }}$ and the Beilinson-Parshin conjecture [Kah05, proof of Theorem 56].

We now give some interesting consequences of Conjecture 4.1 for motives which are truly motives over $\mathbb{Z}$, i.e., not coming from a motive over $\mathbb{F}_{p}$. It would be interesting to know whether other axioms on mixed motives over $\mathbb{Q}$, such as the agreement of homological and numerical equivalence on smooth projective varieties $X_{\eta} / \mathbb{Q}$ can be derived from Conjecture 4.1] 
Theorem 4.5. As in Example [2.8, consider the motive $M=\mathrm{M}(X)\{m-d\}[p-2 m]$, $X / \mathbb{Z}$ regular, flat, projective and of equidimension d. Then Conjecture 4.1 for $M$ is equivalent to the Beilinson-Soulé vanishing conjecture

$$
K_{2 m-p}(X)_{\mathbb{Q}}^{(m)}=0 \quad(\text { for } p<0 \text { and for } p=0, m>0) .
$$

Proof: The group $\widehat{\mathrm{H}}^{0}(M)$ appears in the long exact sequence

$\ldots \rightarrow \mathrm{H}_{\mathrm{D}}^{-1}(M)=\mathrm{H}_{\mathrm{D}}^{2 d-p-1}(X, d-m) \rightarrow \widehat{\mathrm{H}}^{0}(M) \rightarrow \mathrm{H}^{0}(M)=\underbrace{K_{p-2 m}(X)^{(d-m)}}_{=0} \rightarrow \ldots$

where the right hand vanishing is because $p-2 m<0$ for $p<0$ and $p=0, m>0$. The left hand vector space is dual to $\mathrm{H}_{\mathrm{D}}^{p}(X, m)$ by (2.5) (note that $d=\operatorname{dim} X_{\mathbb{C}}+1$ ). It vanishes for $p<0$ for trivial reasons. For $p=0$, the short exact sequence (2.3) gives $\mathrm{H}_{\mathrm{D}}^{0}(X, m)=0$ for $m>0$. Indeed, the Hodge structure on $\mathrm{H}_{\mathrm{dR}}^{0}(X)$ only lies in the $(0,0)$-part of the Hodge diamond, i.e., $F^{m}=0$ for $m>0$. Hence the injectivity of $\mathrm{H}_{\mathrm{B}}^{0}(X, \mathbb{R}(m)) \rightarrow \mathrm{H}_{\mathrm{B}}^{0}(X, \mathbb{C}) \stackrel{\cong}{\rightarrow} \mathrm{H}_{\mathrm{dR}}^{0}\left(X_{\mathbb{C}}\right)$ gives the claim. Therefore Conjecture 4.1 for $M$ is equivalent to $\mathrm{H}_{-2}(M,-1)=\mathrm{H}_{2 m-p-2}(\mathrm{M}(X)\{m-d\},-1)=$ $K_{2 m-p}(X)_{\mathbb{R}}^{(m)}=0$.

Example 4.6. Using the notation of Theorem 4.5 the group $\mathrm{H}_{-2}(M,-1)$ vanishes for $2 m-p<0$. Therefore, 4.1 asserts that the Chern class map

$$
\mathrm{H}^{0}(M)_{\mathbb{R}}=K_{p-2 m}(X)_{\mathbb{R}}^{(d-m)} \rightarrow \mathrm{H}_{\mathrm{D}}^{0}(M)=\mathrm{H}_{\mathrm{D}}^{2 d-p}(X, d-m)
$$

is injective for $p-2 m>0$ and an isomorphism for $p-2 m>1$. In particular, the non-torsion part of higher $K$-theory of $X$ is finitely generated - a weakening of Conjecture 5.1

Proposition 4.7. Assuming the existence of motivic t-structure on $\mathbf{D M}_{\mathrm{b}, c}(\mathbb{Z})$ such that Betti and de Rham realization are exact (see Axiom 1.2(1ii) and (2.14)), the perfectness of the Arakelov intersection pairing for all motives $M \in \mathbf{D M}_{\mathrm{b}, c}(\mathbb{Z})$ implies that the cohomological dimension of mixed motives over $\mathbb{Z}$ is two, i.e., $\operatorname{Hom}(\mathbf{1}[1], M[n])=0$ for any $n>2$ and $M \in \mathbf{M M}(\mathbb{Z})$.

Proof: Let $M$ be a mixed motive. The group $\mathrm{H}_{1-n}(M,-1)_{\mathbb{R}}=\operatorname{Hom}(\mathbf{1}[1-$ $n](-1), M)_{\mathbb{R}}$ is zero for $n<0$ : in this case $\mathbf{1}[1-n]$ lies in degree $n<0$ (with respect to the motivic $t$-structure). On the other hand this group is dual, via $\pi_{M[n-3]}$, to $\widehat{\mathrm{H}}_{\mathbb{R}}^{3-n}(M)$. In (2.17), this group lies between $\mathrm{H}_{\mathbb{R}}^{3-n}(M)$ which vanishes for $n>2$ for the same reason and the Deligne cohomology group $\mathrm{H}_{\mathrm{D}}^{3-n}(M)=\operatorname{Hom}\left(M, \mathrm{H}_{\mathrm{D}}[3-n]\right)$ which in turn vanishes by exactness of Betti and de Rham realization, except for $n=1,2$, as in (2.15). Consequently, $\mathrm{H}_{1-n}(M)=0$ except for $n=0,1,2$.

Lemma 4.8. Under Conjecture 4.1. $\mathrm{H}^{i}(M)$ is nonzero only for finitely many $i \in \mathbb{Z}$.

This is a consequence of the spectral sequence $\mathrm{H}^{a}\left({ }^{\mathrm{p}} \mathrm{H}^{b}(M)\right) \Rightarrow \mathrm{H}^{a+b}(M)$, the boundedness of the motivic $t$-structure and of the cohomological dimension $[\mathrm{Sch} 12 \mathrm{~b}$, Axiom 4.1.]. It also follows from the perfectness of the Arakelov intersection pairing (not using the axioms on mixed motives):

Proof: It suffices to check the claim for $M=\mathrm{M}(X)(m)$, where $X$ is as in Example 4.6 and $m \in \mathbb{Z}$, since these objects generate $\mathbf{D M}_{\mathrm{b}, c}(\mathbb{Z})$ as a thick category by resolution of singularities. Now, the claim follows as in Proposition 4.7 using the vanishing $K_{k}(X)$ for $k<0$ and the vanishing of almost all Deligne cohomology groups of $X$. 


\section{Are special $L$-values given by the Arakelov in- tersection pairing?}

Throughout this section, let $M$ be any geometric motive over $\mathbb{Z}$. In this chapter, wherever ranks of motivic cohomology groups are involved, we assume that the Bass conjecture holds up to torsion:

Conjecture 5.1. For any regular scheme $X / \mathbb{Z}, \operatorname{dim}_{\mathbb{Q}} K_{i}(X)_{\mathbb{Q}}<\infty$.

We need the following consequence (by resolution of singularities): motivic cohomology of all geometric motives over $\mathbb{Z}$ is finitely generated.

By [Sch12b, Axiom 4.1.] (see also Lemma 4.8) and (2.17) only finitely many $\mathrm{H}^{i}(M)$ and $\widehat{\mathrm{H}}^{i}(M)$ are nonzero as $i \in \mathbb{Z}$ varies. Thus, the Euler characteristic

$$
\chi(M):=\sum_{i}(-1)^{i} \operatorname{dim} \mathrm{H}^{i}(M)
$$

and similarly $\widehat{\chi}(M), \chi_{\mathrm{D}}(M)$ are well-defined. We write $\operatorname{det} H^{*}:=\otimes_{i \in \mathbb{Z}} \operatorname{det}^{(-1)^{i}} H^{i}$ for any bounded family $H^{i}$ of finite-dimensional vector spaces, such as $\mathrm{H}^{i}(M)$ etc. The determinant of Arakelov motivic cohomology groups carries a $\mathbb{Q}$-structure by the isomorphism induced by (2.13) and (2.17),

$$
\operatorname{det} \widehat{\mathrm{H}}_{\mathbb{R}}^{*}(M)=\left(\operatorname{det} \mathrm{H}^{*}(M) \otimes \operatorname{det}^{-1} \mathrm{H}_{\mathrm{B}, \mathbb{Q}}^{*}(M) \otimes \operatorname{det} \mathrm{H}_{\mathrm{dR}}^{/ F, \operatorname{alg}, *}(M)\right) \otimes_{\mathbb{Q}} \mathbb{R} .
$$

Conjecture 5.2. The order of the $L$-function of $M$ (Definition 3.1) is given by

$$
\operatorname{ord}_{s=0} L(M, s)=-\chi\left(M^{\vee}(-1)\right) .
$$

As usual, negative orders mean a pole, positive ones a zero of the $L$-function. Moreover, assuming the perfectness of the Arakelov intersection pairings $\pi_{M[k]}$ (Definition 2.5) for all $k \in \mathbb{Z}$ asserted by Conjecture 4.1, the special $L$-value is given by

$$
L^{*}(M, 0) \equiv 1 / \Pi_{M}\left(\bmod \mathbb{Q}^{\times}\right) .
$$

Here $\Pi_{M}$ means the following: the perfectness of the Arakelov intersection pairing yields a map

$$
\operatorname{det} \mathrm{H}_{-2+*}(M,-1)_{\mathbb{R}} \otimes \operatorname{det} \widehat{\mathrm{H}}_{\mathbb{R}}^{*}(M) \rightarrow \mathbb{R} .
$$

The $\mathbb{Q}$-structure on the left maps to a real number denoted $\Pi_{M}$. Note that $\Pi_{M}$ is well-defined up to multiplication by a non-zero rational number.

Notation 5.3. For a projective flat scheme $X / \mathbb{Z}$ with smooth generic fiber $X_{\eta} / \mathbb{Q}$, we write $E:=\eta_{! *} \eta^{*} \mathrm{~h}^{-b}(X,-m) \in \mathbf{M M}(\mathbb{Z})$ and $M_{\eta}=\eta^{*}[-1] E=$ $\mathrm{h}^{-b-1}\left(X_{\eta},-m\right) \in \mathbf{M M}(\mathbb{Q})$. The definition of $E$ is recalled in Section 1.2. In particular, whenever $E$ is considered, we need to assume the axioms on mixed motives mentioned in Section 1.2. The motive $E$ only depends on $X_{\eta}$, not on $X$. It is pure of weight $w:=\operatorname{wt}(E)=2 m-b$. Putting $d:=\operatorname{dim} X$ and $d_{\eta}=\operatorname{dim} X_{\eta}$, the dual

$$
E^{\vee}=\left(\eta_{! *} \eta^{*} \mathrm{~h}^{-2 d+4+b}(X, 1-d+m)\right)[-2]
$$

is pure of weight $-w$, while $M_{\eta}$ is pure of weight $w-1$.

Under Conjecture 4.1, the pole order conjecture is equivalent to

$$
\operatorname{ord}_{s=0} L(M, s)=-\widehat{\chi}(M) .
$$

We expound some structural properties of the conjecture. In order to state the compatibility with the functional equation, we shall need the following conjecture due to Deligne. It implies the compatibility of the $L$-values conjecture for critical pure motives $M_{\eta}$ over $\mathbb{Q}$ (i.e., motives such that $\mathrm{H}_{\mathrm{w}}^{i}\left(M_{\eta}\right)=0, i=0,1$ ) with the functional equation [Del79, Theorem 5.6]. 
Conjecture 5.4. Del79, Conjecture 6.6] Let $M$ be a pure motive over $\mathbb{Q}$ with respect to homological equivalence, i.e., a direct summand in $\mathbf{M}_{\mathrm{hom}}(\mathbb{Q})$ of $h\left(X_{\eta}, m\right)$ where $X_{\eta} / \mathbb{Q}$ is smooth projective. Assume that $M$ is of rank one, that is to say, its Betti realization (or, equivalently, de Rham or $\ell$-adic realization) is one-dimensional. Then $M$ is of the form $M(\epsilon)(n)$, where $n$ is an integer and $\epsilon: \operatorname{Gal}(\mathbb{Q}) \rightarrow \mathbb{Q}^{\times}$is a finite character and $M(\epsilon)$ denotes the Dirichlet motive attached to the one-dimensional representation, $\epsilon$, of $\operatorname{Gal}(\mathbb{Q})$ (loc. cit.).

Theorem 5.5. (i) Conjecture 5.2 is triangulated: given a distinguished triangle $M_{1} \rightarrow M_{2} \rightarrow M_{3}$ in $\mathbf{D M}_{\mathrm{b}, c}(\mathbb{Z})$, the conjecture predicts

$$
L^{*}\left(M_{1}, 0\right) L^{*}\left(M_{3}, 0\right)=L^{*}\left(M_{2}, 0\right)
$$

and additively with the pole orders. In particular, the subcategory of $\mathbf{D M}_{\mathrm{b}, c}(\mathbb{Z})$ of motives for which the conjecture holds is triangulated.

(ii) Assume Deligne's Conjecture 5.4. Conjecture 1.5 $\left(\sim_{\text {rat }}=\sim_{\text {num }}\right)$, the functional equation for completed L-functions over $\mathbb{Q}$ (Conjecture [3.12) and 4.1. Then Conjecture 5.2 for any accessible motive $M$ (Definition [3.8) is equivalent to the one for $M^{\vee}\{-1\}$.

Note that accessible motives generate $\mathbf{D M}_{\mathrm{b}, c}(\mathbb{Z})$ as a thick category (Remark 3.9).

Proof: (1): The pole order additivity is clear. The multiplicativity of the special values formula follows easily by considering the long exact sequences made of $\widehat{\mathrm{H}}_{\mathbb{R}}^{*}\left(M_{i}\right)$ and $\mathrm{H}_{*}(M,-1)_{\mathbb{R}}$. By construction, the $\mathbb{Q}$-structure on Arakelov motivic cohomology is triangulated, i.e., there is a canonical isomorphism $\operatorname{det} \widehat{\mathrm{H}}_{\mathbb{R}}^{*}\left(M_{2}\right)=$ $\operatorname{det} \widehat{\mathrm{H}}_{\mathbb{R}}^{*}\left(M_{1}\right) \otimes \operatorname{det} \widehat{\mathrm{H}}_{\mathbb{R}}^{*}\left(M_{3}\right)$ of $\mathbb{R}$-vector spaces, respecting the $\mathbb{Q}$-structure.

(ii): By Remark 3.9(1ii), it is enough to show the claim for all $M$ contained in the triangulated subcategory of $\mathbf{D M}_{\mathrm{b}, c}(\mathbb{Z})$ generated by the image of $i_{*}: \mathbf{D M}_{\mathrm{b}, c}\left(\mathbb{F}_{p}\right) \rightarrow$ $\mathbf{D M}_{\mathrm{b}, c}(\mathbb{Z})$ for all primes $p$ and motives $E$ as in Notation 5.3 .

We put ord $:=\operatorname{ord}_{s=0}$ and $\chi_{w}^{a}(M):=\sum_{i \in \mathbb{Z}}(-1)^{i} \operatorname{dim} \mathrm{H}_{w}^{a}\left(\mathrm{H}^{i}\left(\mathrm{R}_{\mathrm{H}}(M)\right)\right.$ for $a=$ 0,1 , where $R \Gamma_{\mathrm{H}}$ denotes the Hodge realization functor defined in (2.7). Conjecture 5.2 for $M$, ord $L(M, s)=-\chi\left(M^{\vee}(-1)\right)$, is equivalent to

$$
\begin{aligned}
& \operatorname{ord} \Lambda(M) \stackrel{3.21}{=} \text { ord } L(M)-\chi_{w}^{1}\left(M^{\vee}(-1)\right) \\
& =-\chi\left(M^{\vee}(-1)\right)-\chi_{w}^{1}\left(M^{\vee}(-1)\right) \\
& \stackrel{4.1}{=}-\widehat{\chi}(M)-\chi_{w}^{1}\left(M^{\vee}(-1)\right) \\
& \stackrel{(2.5)}{=}-\chi(M)+\chi_{\mathrm{D}}(M)-\chi_{w}^{0}(M) \\
& =-\chi(M)-\chi_{w}^{1}(M)
\end{aligned}
$$

Indeed, $\chi_{\mathrm{D}}(M)=\chi_{w}^{0}(M)-\chi_{w}^{1}(M)$ (at least) for all $M$ as in the claim: for $M=E$, this follows from (2.4), (2.6), and (2.15), while for $M=i_{*} N$, these terms are zero. By Lemma 3.13, the functional equation for mixed motives over $\mathbb{Q}$ implies the one for motives over $\mathbb{Z}$, so that ord $\Lambda\left(M^{\vee}(-1)\right)=\operatorname{ord} \Lambda(M)$. Again invoking the pole order calculation of $L_{\infty}$-functions we get ord $L\left(M^{\vee}(-1)\right)=-\chi(M)$, that is, the conjectural prediction of the pole order of $L\left(M^{\vee}(-1)\right)$. This settles the compatibility of the pole order prediction with the functional equation.

As for the special $L$-values, the claim is again triangulated. For motives $M=$ $i_{*} N$, where $i:$ Spec $\mathbb{F}_{p} \rightarrow$ Spec $\mathbb{Z}$ and $N$ is any geometric motive over $\mathbb{F}_{p}$ we have $M^{\vee}\{-1\}=i_{*} N^{\vee}$. The functional equation reads $L\left(i_{*} N, s\right)=a b^{s} L\left(i_{*} N^{\vee},-s\right)$, with $a$ and $b$ in $\mathbb{Q}^{\times}$(Remark 3.14, this uses the agreement of numerical and homological 
equivalence, implied by Conjecture 1.5) On the other hand $\Pi_{i_{*} N} \equiv \Pi_{i_{*} N^{\vee}} \bmod \mathbb{Q}^{\times}$ by Example 2.7

To check the claim for $M=E$ as above, we may assume $X$ is of equidimension $d$. Each individual Deligne cohomology group $\mathrm{H}_{\mathrm{D}}^{r}(E)$ carries the $\mathbb{Q}$-structure mentioned in (2.2). We can assume $w:=\operatorname{wt}(E)=2 m-b \leq 2$, since otherwise we can replace $E$ by $E^{\vee}(-1)[2]$. Let $M_{\eta}:=\eta^{*} M[-1]=\mathrm{h}^{-b-1}\left(X_{\eta}, m\right)$, where $X_{\eta}$ is the generic fiber of $X$. For $w=2$, the hard Lefschetz axiom implies an isomorphism $E \cong E^{\vee}(-1)[2]$ (see (5.10) $)$, so that there is nothing to show in that case. Let now $w \leq 1$. We write $L^{*}(-):=L^{*}(-, 0)$. Deligne's conjecture 5.4 implies (see loc. cit.)

$$
\frac{L^{*}(E)}{L^{*}\left(E^{\vee}(-1)\right)} \stackrel{\sqrt[3.5]{=}}{=} \frac{L^{*}\left(M_{\eta}^{\vee}(-1)\right)}{L^{*}\left(M_{\eta}\right)} \stackrel{5.4}{=} \frac{a_{1}}{a_{2}} \bmod \mathbb{Q}^{\times}
$$

where $a_{1}$ is an element in the $\mathbb{Q}$-lattice of $\operatorname{det} \mathrm{H}_{\mathrm{D}}^{0}\left(E^{\vee}(-1)\right)(=\mathbb{R})$ given by the $\mathbb{Q}$ structure on this Deligne cohomology group, and $a_{2}$ is an element in the $\mathbb{Q}$-lattice of $\operatorname{det}^{-1} \mathrm{H}_{\mathrm{D}}^{1}(E)$, regarded as an element of $\operatorname{det} \mathrm{H}_{\mathrm{D}}^{0}\left(E^{\vee}(-1)\right)$ using the isomorphism $\mathrm{H}_{\mathrm{D}}^{1}\left(M_{\eta}^{\vee}(-1)\right)^{\vee} \rightarrow \mathrm{H}_{\mathrm{D}}^{0}\left(M_{\eta}\right)$, cf. (2.5). In other words, the isomorphism $\operatorname{det} \mathrm{H}_{\mathrm{D}}^{1}(E) \rightarrow$ $\operatorname{det} \mathrm{H}_{\mathrm{D}}^{0}\left(E^{\vee}(-1)\right)$ is multiplication by $a_{1} / a_{2}$ with respect to the $\mathbb{Q}$-structures on both sides.

For $r \neq 1$, the group $\mathrm{H}_{\mathrm{D}}^{r}(E)$ and its $\mathbb{Q}$-structure is trivial, since the corresponding Betti and (truncated) de Rham cohomology groups vanish. Therefore there is a canonical isomorphism

$$
\operatorname{det} \mathrm{H}_{\mathrm{D}}^{*}(E) \cong \operatorname{det}^{-1} \mathrm{H}_{\mathrm{D}}^{1}(E)
$$

(including the $\mathbb{Q}$-structure). Thus

$$
\begin{aligned}
\operatorname{det} \widehat{\mathrm{H}}_{\mathbb{R}}^{*}(E) \otimes \operatorname{det} \mathrm{H}_{*}(E,-1)_{\mathbb{R}} & =\operatorname{det}^{*}(E)_{\mathbb{R}} \otimes \operatorname{det}^{-1} \mathrm{H}_{\mathrm{D}}^{*}(E) \otimes \operatorname{det} \mathrm{H}^{*}\left(E^{\vee}, 1\right)_{\mathbb{R}} \\
& \cong \operatorname{det} \mathrm{H}^{*}(E)_{\mathbb{R}} \otimes \operatorname{det}^{-1} \mathrm{H}_{\mathrm{D}}\left(E^{\vee}(-1)\right) \otimes \operatorname{det} \mathrm{H}^{*}\left(E^{\vee}, 1\right)_{\mathbb{R}} \\
& =\operatorname{det} \mathrm{H}_{*}\left(E^{\vee}\right)_{\mathbb{R}} \otimes \operatorname{det} \widehat{\mathrm{H}}_{\mathbb{R}}^{*}\left(E^{\vee}, 1\right)
\end{aligned}
$$

Both the left hand side and the last term on the right hand side map to $\mathbb{R}$ via the Arakelov intersection pairings for $E$ and $E^{\vee}\{-1\}$, respectively. The two pairings are compatible with the isomorphism by the commutativity of (2.20). By Conjecture 5.2 for $E$, the image of the $\mathbb{Q}$-structure on the left hand side is $L^{*}(E)^{-1}$, while the one from the right hand side is, by 5.2, just $1 / L^{*}\left(E^{\vee}(-1)\right)$. Hence the two cases of the conjecture are equivalent.

In the remainder of this paper, we show how certain special cases of 5.2 are related to conjectures of Beilinson, Soulé, and Tate. In order to formulate our main result as succinctly as possible, we formulate the following

Conjecture 5.6. For the motive $E$ defined in Notation 5.3 with $w:=\operatorname{wt}(E)=$ 2 , the Arakelov intersection pairing $\pi_{E[-2]}: \mathrm{H}_{0}(E,-1) \times \widehat{\mathrm{H}}^{2}(E) \rightarrow \mathbb{R}$ agrees with Beilinson's height pairing (5.6).

Remark 5.7. By Theorem 1.3 and (2.15), we know $\mathrm{H}_{0}(E,-1)=\mathrm{CH}^{d-m}\left(X_{\eta}\right)_{\mathbb{Q}}$ and $\widehat{\mathrm{H}}^{2}(E)=\mathrm{H}^{2}(E)=\mathrm{CH}^{m}\left(X_{\eta}\right)_{\mathbb{Q}}$ (cf. the proof of Proposition 5.16), so this conjecture only concerns the pairing itself. Moreover, (5.6) is induced by the Gillet-Soulé intersection pairing

$$
\mathrm{CH}^{d-m}(X)_{\mathbb{Q}} \times \widehat{\mathrm{CH}}^{m}(X)_{\mathbb{Q}} \rightarrow \widehat{\mathrm{CH}}^{d}(X)_{\mathbb{Q}} \stackrel{f_{*}}{\longrightarrow} \widehat{\mathrm{CH}}^{1}(\mathbb{Z})=\mathbb{R},
$$

which in turn is induced by (4.2). As mentioned in Example 2.8, this pairing agrees with the Arakelov intersection pairing for $\mathrm{M}(X)\{-m\}$ at least up a $\mathbb{Q}$-linear combination of $\log p_{i}$, where $p_{i}$ are the primes such that the restriction of $X$ is 
smooth over $\mathbb{Z}\left[1 / \prod p_{i}\right]$. It is worth mentioning that this comparison is an entirely formal consequence of the use of stable homotopy category. Its definition as the homotopy category of spectra of simplicial presheaves on smooth schemes yields immediate comparison results such as [Sch12a, Thm. 7.4] for smooth schemes, but not easily for other schemes. Therefore, it is a natural idea to overcome this hurdle by studying (Arakelov) motivic cohomology for log-smooth schemes. By de Jong's resolution of singularities, motives of all $\log$-smooth $\mathbb{Z}$-schemes should generate a category of motives of logarithmic schemes over $\mathbb{Z}$. This would allow to bypass Conjecture 5.6. I plan to return to this question in a subsequent paper.

The following two theorems summarize the remainder of this paper: under standard assumptions on motives and their $L$-functions, it shows that Beilinson's, Soulé's, and Tate's conjectures are essentially equivalent to the conceptual reformulation made possible by the use of the Arakelov intersection pairing.

Theorem 5.8. The following are equivalent:

(i) The conjecture of Soulé (5.11), restricted to regular, projective (but not necessarily flat) schemes.

(ii) The restriction of the pole order formula (Conjecture 5.2) to the category of easily accessible motives (Definition 3.8).

Proof: This follows immediately from Theorem 5.12 by Theorem 5.5 .

By Remark 3.9, the thick closure of the category of easily accessible motives is the entire category $\mathbf{D M}_{\mathrm{b}, c}(\mathbb{Z})$. Thus, the pole order formula of Conjecture 5.2 can be regarded as an extension of Soulé's conjecture to direct summands.

Theorem 5.9. We assume the existence of mixed motives as formulated in Axiom 1.2 and the agreement of Beilinson's height pairing with the Arakelov intersection pairing (Conjecture 5.6). Moreover, in order to incorporate the compatibility of $L$ values with respect to the functional equation, we assume Deligne's conjecture 5.4 on rank one motives, and the functional equation for completed L-functions over $\mathbb{Q}$ (Conjecture 3.12). Finally, we assume that the pole order formula of Conjecture 5.2 holds for all motives in $\mathbf{D M}_{\mathrm{B}, c}(\mathbb{Z})$.

Then, the following are equivalent:

(i) The conjunction of the conjectures of Beilinson (L-values and $\sim_{\text {num }}=\sim_{\text {rat }}$, 5.14, 1.5), and Tate (5.19).

(ii) The restriction of the conjunction of the perfectness of the Arakelov intersection pairings (Conjecture 4.1) and the special L-values formula (Conjecture 5.2) to the subcategory $\mathbf{D} \mathbf{M}_{\mathrm{b}, \operatorname{tr}}(\mathbb{Z}) \subset \mathbf{D M}_{\mathrm{b}, c}(\mathbb{Z})$ of accessible motives (Definition 3.8).

Proof: By Remark 3.9 (iii), $\mathbf{D M}_{\mathrm{b}, \mathrm{tr}}(\mathbb{Z})$ is contained in the triangulated category generated by motives $M=E$ as in Notation 5.3, and motives of the form $M=i_{*} N$, $N \in \mathbf{D M}_{\mathrm{b}, c}\left(\mathbb{F}_{p}\right), i:$ Spec $\mathbb{F}_{p} \rightarrow$ Spec $\mathbb{Z}$. For the latter type of motives, Conjecture 4.1 is equivalent to Conjecture 1.5 by Theorem 4.3 and 5.2 is equivalent to the Tate conjecture by Theorem 5.20 .

The subcategory of $\mathbf{D M}_{\mathrm{B}, c}(\mathbb{Z})$ of motives $M$ for which all pairings $\pi_{M[k]}$ are perfect is triangulated since motivic and Arakelov motivic cohomology behave well under triangles. Moreover, 4.1 for $M\left(\in \mathbf{D M}_{\mathrm{b}, \operatorname{tr}}(\mathbb{Z})\right)$ is equivalent to 4.1 for $M^{\vee}\{-1\}$ by Remark 4.2(ii). In a similar vein, Conjecture 5.2 is stable under distinguished triangles, and 5.2 for $M$ is equivalent to 5.2 for $M^{\vee}\{-1\}$ (Theorem 5.5). 
To finish (ii) $\Rightarrow$ (iii), using the calculation of $E^{\vee}\{-1\}$ in (5.2), we therefore only need to consider $M=E$ with $w:=\operatorname{wt}(E)=2 m-b \leq 2$. Beilinson's pole order conjecture for $M_{\eta}, 5.14(\mathrm{~A})$, is equivalent (see (5.14) $)$ to

$$
\operatorname{ord}_{s=0} L(E, s)=-\chi\left(E^{\vee}(-1)\right)+\operatorname{dim} H^{1}\left(E^{\vee}(-1)\right) .
$$

By assumption, $L(E, s)=-\chi\left(E^{\vee}\{-1\}\right)=-\chi\left(E^{\vee}(-1)\right)$, so that we get $\mathrm{H}^{1}\left(E^{\vee}(-1)\right)=$ 0 . Using this vanishing, part (B) of Beilinson's conjecture is equivalent to the perfectness of the intersection pairings $\pi_{E[k]}, k \in \mathbb{Z}$ (with $w=\operatorname{wt}(E) \leq 2$ ), by Proposition 5.16. This shows that 1.5, 5.11 and 5.14(B) together imply 4.1 for all $M \in \mathbf{D M}_{\mathrm{b}, \mathrm{tr}}(\mathbb{Z})$. Then parts $(\mathrm{A}),(\mathrm{C})$ of Beilinson's conjecture are equivalent to 5.2 for all motives of the form $E$ (of weight $\leq 2$ ), by Theorem 5.18 .

The converse implication (iii) $\Rightarrow$ (ii) is shown using the same arguments.

Remark 5.10. It is natural to ask for the equivalence of the following two statements:

(i) The conjectures of Beilinson, Soulé, and Tate (5.14 1.5, 5.11, 5.19).

(ii) The restriction of Conjectures 4.1 and 5.2 to the category of accessible motives.

Under the assumptions of 5.9, except for the pole order formula assumption, the above proof does show (iii) $\Rightarrow$ (ii). The latter addditional assumption is only needed to prove the converse, and is actually only needed for motives of the form $M=E$ as above. Moreover, it holds unconditionally if $\mathrm{M}\left(X_{\eta}\right)$ is an Artin-Tate motive (Theorem 1.4). The vanishing $\mathrm{H}^{1}\left(E^{\vee}(-1)\right)=0$ also follows from the Soulé+Tate conjecture if one can show $E \in \mathbf{D M}_{\mathrm{b}, \mathrm{tr}}(\mathbb{Z})$, which in its turn would follow if the motivic $t$-structure on $\mathbf{D M}_{\mathrm{b}, c}(\mathbb{Z})$ restricts to a $t$-structure on $\mathbf{D M}_{\mathrm{b}, \mathrm{tr}}(\mathbb{Z})$. In this case, the proof of [Sch12b, Prop. 5.6] referred to in Remark 3.9)(iii) could be adapted to $\mathbf{D M}_{\mathrm{b}, \mathrm{tr}}(\mathbb{Z})$.

\subsection{Relation to a conjecture of Soulé}

Conjecture 5.11. (Soulé, Sou84, Conjecture 2.2.]) Let $Y / \mathbb{Z}$ be quasiprojective. Let $m \in \mathbb{Z}$ be arbitrary. Then

$$
\operatorname{ord}_{s=m} \zeta(Y, s)=\sum_{i \geq 0}(-1)^{i+1} \operatorname{dim}_{\mathbb{Q}} K_{i}^{\prime}(Y)_{(m), \mathbb{Q}}
$$

We refer to loc. cit. for the definition of the Adams eigenspace $K_{i}^{\prime}(Y)_{(m), \mathbb{Q}}$. For $Y$ regular, it agrees with $K_{i}(Y)_{\mathbb{Q}}^{(\operatorname{dim} Y-m)}$.

Soulé's conjecture extends a previous conjecture of Tate [Tat65, p. 105]. A formally similar conjecture was also expressed by Lichtenbaum Lic84. The right hand side of (5.5) makes sense under the Bass conjecture 5.1 and the vanishing of almost all $K^{\prime}$-groups, which in turn is a consequence of [Sch12b, Axiom 4.1.]. See also Lemma 4.8 As the thick closure of $\mathbf{D M}_{\mathrm{b}, \mathrm{tr}}(\mathbb{Z})$ is all of $\mathbf{D M}_{\mathrm{b}, c}(\mathbb{Z})$, the following statement can be paraphrased by saying that Soulé's conjecture is essentially equivalent to the pole order part of Conjecture 5.2. This proof does not make use of mixed motives.

Theorem 5.12. Conjecture 5.11 for $Y$ and $m$ is equivalent to the pole order prediction of Conjecture 5.2 for $M=\mathrm{M}_{\mathrm{c}}(Y)(-m)$.

Proof: Proposition 3.7 says $\zeta(Y, s+m)=L\left(\mathrm{M}_{\mathrm{c}}(Y)(-m), s\right)$. The statement for $Y$ is implied by the conjunction of the one for some open subscheme $U$ of $Y$ and $Z:=$ $Y \backslash U$, since Adams eigenspaces in $K^{\prime}$-theory have a localization sequence [Sou84, 
1.3.], and motives with compact support behave well: $\mathrm{M}_{\mathrm{c}}(Z) \rightarrow \mathrm{M}_{\mathrm{c}}(Y) \rightarrow \mathrm{M}_{\mathrm{c}}(U)$ is a distinguished triangle. In particular we may assume that $Y$ is integral. Thus, there is an open affine subscheme $U$ of $Y$ that is either smooth over $\mathbb{Z}$ or over some $\mathbb{F}_{p}$ : if $Y / \mathbb{Z}$ is flat, one can take an open neighborhood of a smooth point of the generic fiber of $Y$, otherwise $Y$ lies over some Spec $\mathbb{F}_{p}$ and one can take a neighborhood of a smooth point of $Y$. Let $f: Y \rightarrow \mathbb{Z}$ be the projection. By Noetherian induction, we may replace $Y$ by $U$ and hence assume $Y$ is regular and affine of dimension $d$, so that $\left(\mathrm{M}_{\mathrm{c}}(Y)(-m)\right)^{\vee}\{-1\}=f_{!} f^{*} \mathbf{1}(m)\{-1\}=f_{!} f^{!} \mathbf{1}(m)\{-d\}$ by purity. Hence

$$
\begin{aligned}
\chi\left(\left(\mathrm{M}_{\mathrm{c}}(Y)(-m)\right)^{\vee}\{-1\}\right) & =\chi(\mathrm{M}(Y)(m-d)[-2 d]) \\
& =\sum_{i \in \mathbb{Z}}(-1)^{i} \operatorname{dim} \mathrm{H}^{i+2 d}(Y, m-d) \\
& =\sum_{i \in \mathbb{Z}}(-1)^{i} \operatorname{dim} K_{i}(Y)_{\mathbb{Q}}^{(d-m)} .
\end{aligned}
$$

Example 5.13. We continue Examples 2.8 and 4.6 and look at the special values of the $\zeta$-function of $X$ : by Proposition 3.7 we have $L(M, s)=\zeta(X, s+d-m)$. The Arakelov intersection pairing $\pi_{M[i]}$ concerns the following groups

$$
\begin{array}{cccc}
0 & \times & 0 & i \leq-1 \\
K_{0}(X)_{\mathbb{R}}^{(m)} & \times & \widehat{\mathrm{H}}_{\mathbb{R}}^{0}(M) & i=0 \\
K_{1}(X)_{\mathbb{R}}^{(m)} & \times & \operatorname{coker} K_{0}(X)_{\mathbb{R}}^{(d-m)} \rightarrow \mathrm{H}_{\mathrm{D}}^{2(d-m)}(X, d-m) & i=1 \\
K_{i}(X)_{\mathbb{R}}^{(m)} & \times & \mathrm{H}_{\mathrm{D}}^{2(d-m)+i-1}(X, d-m) & i>1 .
\end{array}
$$

The pairing for $i \geq 1$ is given by the Chern class $K_{i}(X)_{\mathbb{R}}^{(m)} \rightarrow \mathrm{H}_{\mathrm{D}}^{2 m-i}(X, m)$ together with the cup product on Deligne cohomology, followed by the push-forward $f_{*}$ : $\mathrm{H}_{\mathrm{D}}^{2 d-1}(X, d) \rightarrow \mathrm{H}_{\mathrm{D}}^{1}(U, 1)=\mathbb{R}$. I expect that the group $\widehat{\mathrm{H}}_{\mathbb{R}}^{0}(M)$ is isomorphic to $\mathrm{CH}^{d-m}(X)_{\mathbb{R}}$ and that the pairing $\pi_{M}$ is the natural pairing of (arithmetic) Chow groups (cf. Remark 5.7). We do know that these two pairings agree up to a $\mathbb{Q}$-linear combination of $\log \prod p_{i}$, where $p_{i}$ are the primes such that the restriction of $X$ is smooth over $\mathbb{Z}\left[1 / \prod p_{i}\right]$.

These pairings assemble to a map

$$
\bigotimes \pi_{M[i]}: \bigotimes_{i} \operatorname{det}^{(-1)^{i}}\left(\mathrm{H}_{-2-i}(M,-1)_{\mathbb{R}} \otimes \widehat{\mathrm{H}}_{\mathbb{R}}^{i}(M)\right) \stackrel{\cong}{\rightrightarrows} \mathbb{R} .
$$

(Even though the groups $\widehat{\mathrm{H}}^{i}(M)$ vanish for $i<0$, the determinant carries a nontrivial information related to these groups, namely the determinants of the Chern class map, see (4.4).) Conjecture 5.2 asserts that-modulo $\mathbb{Q}^{\times}-L^{*}(M, 0)$ is the reciprocal of the image of 1 in $\mathbb{R}$ via the $\mathbb{Q}$-structure map of the left hand term. The class number formula has been interpreted in terms similar to the one above, see [Sou92, III.4.3].

\subsection{Relation to Beilinson's conjecture}

In this section, we use the notation of 5.3. The following is Beilinson's conjecture Beis4, Beis6. Part (A) concerns the pole order of $L$-functions, part (B) is about the relation of Deligne cohomology and motivic cohomology, and (C) expresses the special $L$-value up to $\mathbb{Q}^{\times}$in terms of determinants of the isomorphisms asserted in (B). The pole order conjecture in case $w=3$ is due to Tate [Tat65.

Conjecture 5.14. Let $X_{\eta} / \mathbb{Q}$ be smooth and projective. 
(A)

$\operatorname{ord}_{s=m} L_{\mathbb{Q}}\left(\mathrm{h}^{-b-1}\left(X_{\eta}\right), s\right)=\operatorname{ord}_{s=0} L_{\mathbb{Q}}\left(M_{\eta}, s\right)= \begin{cases}0 & w \geq 4 \\ -\operatorname{dim} \mathrm{CH}^{n}\left(X_{\eta}\right)_{\mathbb{Q}} / \text { hom } & w=3 \\ \operatorname{dim} \mathrm{CH}^{n}\left(X_{\eta}\right)_{\mathbb{Q}, \text { hom }} & w=2 \\ \operatorname{dim~H} \mathrm{H}^{b+2}\left(X_{\eta}, n\right)_{\mathbb{Z}} & w \leq 1\end{cases}$

Here $n:=b+2-m=m+2-w$, and the groups at the right have been defined in Section 1.2 ,

(B) For $w=2$, the height pairing

$$
\mathrm{CH}^{m}\left(X_{\eta}\right)_{\mathbb{Q}, \text { hom }} \otimes \mathrm{CH}^{d-m}\left(X_{\eta}\right)_{\mathbb{Q}, \text { hom }} \rightarrow \mathbb{R}
$$

is perfect.

For $w=1$, the map

$$
r_{\infty}:\left(\mathrm{CH}^{m}\left(X_{\eta}\right)_{\mathbb{Q}} / \text { hom } \oplus \mathrm{H}^{2 m+1}\left(X_{\eta}, n\right)_{\mathbb{Z}}\right) \otimes_{\mathbb{Q}} \mathbb{R} \rightarrow \mathrm{H}_{\mathrm{D}}^{2 m+1}(X, n) .
$$

obtained by the composition

$$
\mathrm{CH}^{m}\left(X_{\eta}\right)_{\mathbb{Q}} / \mathrm{hom} \otimes \mathbb{R} \rightarrow \mathrm{H}_{\mathrm{dR}}^{2 m}\left(X_{\mathbb{R}}\right) \rightarrow \mathrm{H}_{\mathrm{D}}^{2 m+1}(X, n)
$$

(see (2.2) for the right hand map) and the realization map, is an isomorphism. For $w \leq 0$, the statement is the same, except that (5.7) gets replaced by

$$
r_{\infty}: \mathrm{H}^{b+2}\left(X_{\eta}, n\right)_{\mathbb{Z}} \otimes_{\mathbb{Q}} \mathbb{R} \rightarrow \mathrm{H}_{\mathrm{D}}^{b+2}\left(X_{\eta}, n\right) .
$$

(C) The special $L$-value $L^{*}\left(M_{\eta}, 0\right)$ is conjecturally given up to a nonzero rational multiple by the following:

For $w=2$, by the determinant of the height pairing (5.6) multiplied with the period of $M_{\eta}$, that is to say, the determinant of the isomorphism

$$
\alpha_{M_{\eta}}: \mathrm{H}^{2 m-1}\left(X_{\eta}(\mathbb{C}), \mathbb{R}(m)\right)^{(-1)^{m}} \rightarrow \mathrm{H}_{\mathrm{dR}}^{2 m-1}\left(\left(X_{\eta}\right)_{\mathbb{R}}\right) / F^{m}
$$

with respect to the usual $\mathbb{Q}$-structures on both sides (compare (2.2)).

For $w=1$, the $L$-value is given, $\bmod \mathbb{Q}^{\times}$, by $d_{\infty}(1)$, where

$d_{\infty}:=\operatorname{det} r_{\infty}: \operatorname{det}\left(\mathrm{H}^{b}\left(X_{\eta}, m\right)_{\mathbb{Z}} \oplus \mathrm{CH}^{m}\left(X_{\eta}\right) / \mathrm{hom}\right)_{\mathbb{R}}=\mathbb{R} \rightarrow \operatorname{det} \mathrm{H}_{\mathrm{D}}^{b}(X, n)=\mathbb{R}$,

the left hand term is endowed with the obvious $\mathbb{Q}$-structure, the right one gets the one stemming from the identification of $\mathrm{H}_{\mathrm{D}}^{b}\left(X_{\eta}, n\right)=\mathrm{H}_{\mathrm{w}}^{1}\left(\mathrm{H}^{b-1}(X, \mathbb{Q}(n))\right)$ with the dual of $\mathrm{H}_{\mathrm{w}}^{0}\left(\mathrm{H}^{b-1}(X, \mathbb{Q}(n))^{\vee}(1)\right)$.

For $w \leq 0$, the statement is the same, except that the term $\mathrm{CH}^{m}\left(X_{\eta}\right) /$ hom is omitted.

This concludes the statement of Beilinson's conjecture. It predicts $L$-values of motives $\mathrm{h}^{-b-1}\left(X_{\eta}, m\right)$ with $w=2 m-b \leq 2$, up to a nonzero rational factor. The remaining weights are adressed by the functional equation (Conjecture 3.12).

We compare Beilinson's conjecture with Conjecture 4.1 and 5.2 applied to the generic intermediate extension $E:=\eta_{! *} \eta^{*} \mathrm{~h}^{-b}(X,-m)$, where $X$ is any projective model of $X_{\eta}$ (see Notation 5.3).

Recall from And04, 5.4.2.1] that the agreement of homological and numerical equivalence (which is part of Axiom 1.2) implies the hard Lefschetz isomorphism:

$$
\mathrm{h}^{-b-1}\left(X_{\eta}, m-b-2\right) \stackrel{\cong}{\longrightarrow} \mathrm{h}^{-2 d_{\eta}+b+1}\left(X_{\eta},-d_{\eta}+m-1\right)=M_{\eta}^{\vee}(-1) .
$$

For $b+1 \leq d_{\eta}$ the map is given by the $\left(d_{\eta}-b-1\right)$-st power of cup product with a hyperplane section, with respect to some embedding $X_{\eta} \subset \mathbb{P}_{\mathbb{Q}}^{N}$. The right hand term of (5.9) is $M_{\eta}^{\vee}(-1)$ by relative purity, applied to the smooth map $X_{\eta} / \mathbb{Q}$. 
Lemma 5.15. The hard Lefschetz isomorphism (5.9) yields an isomorphism

$$
E^{\vee}(-1)[2]=E(m-n)=E(w-2) .
$$

It induces isomorphisms of motivic and Deligne cohomology groups (respecting the $\mathbb{Q}$-structure of the latter):

$$
\begin{aligned}
& \mathrm{CH}^{m}\left(X_{\eta}\right)_{\mathbb{Q}} / \text { hom } \cong \mathrm{CH}^{d-m-1}\left(X_{\eta}\right)_{\mathbb{Q}} / \text { hom, } \\
& \mathrm{CH}^{m}\left(X_{\eta}\right)_{\mathbb{Q}, \text { hom }} \cong \mathrm{CH}^{d-m}\left(X_{\eta}\right)_{\mathbb{Q} \text {,hom } \text { Bei87 } \text { Conj. 5.3.(a)], }} \\
& \mathrm{H}^{b}\left(X_{\eta}, b-m\right)_{\mathbb{Z}} \cong \mathrm{H}^{2 d-b}\left(X_{\eta}, d-m\right)_{\mathbb{Z}} \text { for } w=2 m-b \leq 1 \\
& \mathrm{H}_{\mathrm{D}}^{b}\left(X_{\eta}, b-m\right) \cong \mathrm{H}_{\mathrm{D}}^{2 d-b}\left(X_{\eta}, d-m\right) \text { for } w \leq 1 .
\end{aligned}
$$

Proof: (5.10) is obtained from (5.9) by applying $\eta_{! *}[1]$. Now apply Theorem 1.3 and the calculation of Deligne cohomology in (2.15).

The following proposition compares the perfectness of certain Arakelov intersection pairings with the statements in part $(\mathbb{B})$ in Beilinson's conjecture.

Proposition 5.16. Let $E$ be as in Notation 5.3 with weight $w=\operatorname{wt}(E) \leq 2$. If the weight of $E$ is 2, we assume Conjecture 5.6. The following are equivalent:

(i) The pairings $\pi_{E[i]}$ and $\pi_{E^{\vee}\{-1\}[i]}(i \in \mathbb{Z})$ are perfect.

(ii) Part (B) of Beilinson's conjecture and $\mathrm{H}^{3}\left(E^{\vee}(-1)[2]\right)=\mathrm{H}^{1}\left(E^{\vee}, 1\right)=0$ (only needed if $w \leq 1$ ).

Remark 5.17. The group $\mathrm{H}^{1}\left(E^{\vee}, 1\right)$ vanishes unconditionally if $X_{\eta}$ is such that $M_{\eta}$ is a mixed Artin-Tate motive over $\mathbb{Q}$ (as opposed to a general mixed motive) by Theorem [1.4. Recall from Theorem 1.3 that $\mathrm{H}^{3}(E)=0$ for $w:=\operatorname{wt}(E) \leq 2$.

Proof: The proof combines the hard Lefschetz isomorphism (Lemma 5.15) and the calculation of motivic and Deligne cohomology of $E$ and $E^{\vee}(-1)$ (Theorem 1.3. (2.15)).

The map $\mathrm{H}^{b+2}\left(X_{\eta}, n\right)_{\mathbb{Z}} \rightarrow \mathrm{H}_{\mathrm{D}}^{b+2}\left(X_{\eta}, n\right)$ featuring in (5.7), (5.8) in the cases $w \leq 1$ of Conjecture 5.14 is the Chern class map $\mathrm{H}^{2}(E(m-n)) \rightarrow \mathrm{H}_{\mathrm{D}}^{2}(E(m-n))$. Via hard Lefschetz, this is the same as the Chern class map

$$
\operatorname{ch}\left(E^{\vee}(-1)\right): \mathrm{H}^{0}\left(E^{\vee}(-1)\right) \rightarrow \mathrm{H}_{\mathrm{D}}^{0}\left(E^{\vee}(-1)\right) .
$$

Consider the case $w=1$. By Fontaine's reformulation [Fon92, 9.5], the map (5.7) being an isomorphism is equivalent to the existence of an exact sequence whose right hand map is the composition of the Poincaré duality isomorphism $\phi$ stemming from (2.5), the hard Lefschetz isomorphism and the Chern class map.

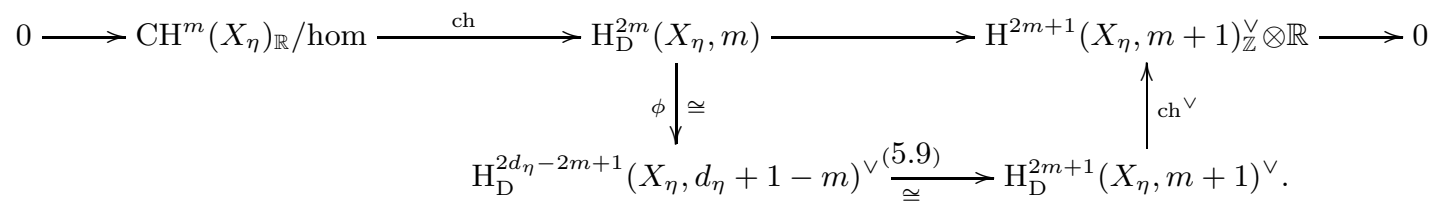

In terms of motivic and Deligne cohomology groups, it reads

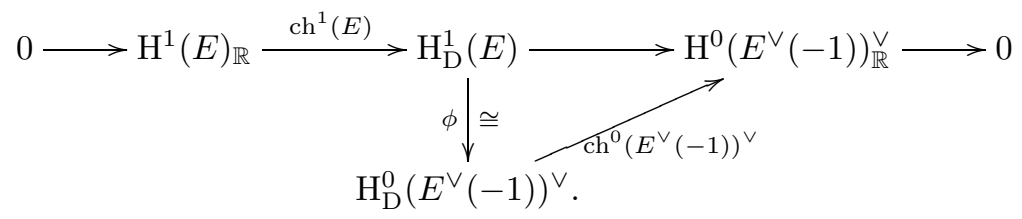


These groups also occur in the following exact sequences, whose terms are paired by the pairings indicated on top:

$$
\begin{array}{cccccccccc}
\pi_{E[-1]}: & & \pi_{E^{\vee}(-1)[-1]}: & & & & & \pi_{E[-2]}: & & \pi_{E^{\vee}(-1)}: \\
\widehat{\mathrm{H}}_{\mathbb{R}}^{1}(E) & \rightarrow & \mathrm{H}^{1}(E)_{\mathbb{R}} & \rightarrow & \mathrm{H}_{\mathrm{D}}^{1}(E) & \rightarrow & \widehat{\mathrm{H}}_{\mathbb{R}}^{2}(E) & \rightarrow & \mathrm{H}^{2}(E)_{\mathbb{R}} \\
\times & & \times & & \times & & \times & & & \times \\
\mathrm{H}^{1}\left(E^{\vee}, 1\right)_{\mathbb{R}} & \leftarrow & \widehat{\mathrm{H}}_{\mathbb{R}}^{1}\left(E^{\vee}, 1\right) & \leftarrow & \mathrm{H}_{\mathrm{D}}^{0}\left(E^{\vee}, 1\right) & \leftarrow & \mathrm{H}^{0}\left(E^{\vee}, 1\right)_{\mathbb{R}} & \leftarrow & \widehat{\mathrm{H}}_{\mathbb{R}}^{0}\left(E^{\vee}, 1\right)
\end{array}
$$

We have $\mathrm{H}^{2}(E)=0$, so the injectivity of $\operatorname{ch}^{0}\left(E^{\vee}(-1)\right)$ is equivalent to $\pi_{E^{\vee}(-1)}$ being perfect. The identification of $\operatorname{coker}^{1} \operatorname{ch}^{1}(E)$ with $\mathrm{H}^{0}\left(E^{\vee}, 1\right)_{\mathbb{R}}^{\vee}$ of $(5.12)$ is equivalent to $\pi_{E[-2]}$ being perfect. The Chern class map $\mathrm{H}^{1}(E)_{\mathbb{R}}=\mathrm{CH}^{m}\left(X_{\eta}\right) /$ hom $\rightarrow \mathrm{H}_{\mathrm{D}}^{1}(E)=$ $\mathrm{H}_{\mathrm{D}}^{2 m}\left(X_{\eta}, m\right) \subset \mathrm{H}_{\mathrm{B}}^{2 m}\left(X_{\eta}, \mathbb{R}(m)\right)$ is injective by definition of homological equivalence. Hence $\widehat{\mathrm{H}}_{\mathbb{R}}^{1}(E)=0$ so that $\mathrm{H}^{1}\left(E^{\vee}, 1\right)=0$ is equivalent to $\pi_{E[-1]}$ being perfect. By the five lemma, $\pi_{E^{\vee}(-1)[-1]}$ is then perfect, too. All other Deligne, motivic, and hence Arakelov motivic cohomology groups of $E^{\vee}(-1)$ and $E$, except for the ones displayed above, vanish.

The case $w<1$ is done similarly: in addition to the above, we have $\mathrm{H}^{1}(E)=0$. Accordingly, (5.12) reduces to an isomorphism $\mathrm{H}_{\mathrm{D}}^{1}(E) \stackrel{\cong}{\rightrightarrows} \mathrm{H}^{0}\left(E^{\vee}(-1)\right)_{\mathbb{R}}^{\vee}$. The details are omitted.

For $w=2$, all Deligne cohomology $\mathrm{H}_{\mathrm{D}}^{*}(E)$ and $\mathrm{H}_{\mathrm{D}}^{*}\left(E^{\vee}, 1\right)$ vanish for weight reasons. Moreover $\mathrm{H}^{a}(E)=\mathrm{H}^{a-2}\left(E^{\vee}(-1)\right)=0$ for $a \neq 2$, so that $\pi_{E^{\vee}(-1)[-1]}$ and $\pi_{E[-1]}$ are perfect. The height pairing (5.6) is just $\pi_{E[-2]}$ according to Conjecture 5.6. Its perfectness is equivalent to the one of $\pi_{E^{\vee}(-1)}$.

Theorem 5.18. We assume the perfectness of the Arakelov intersection pairing for motives of the form $M=E[n]$, with $E$ as in Notation 5.3 and $n \in \mathbb{Z}$. We also assume Conjecture 5.6 if $E$ is of weight 2. Then Beilinson's conjecture (parts (A), (C)) for $M_{\eta}$ is equivalent to Conjecture 5.2 for $E$.

Proof: By hard Lefschetz (Lemma 5.15) and calculation of motivic cohomology of $E$, Theorem 1.3, part (A) of Beilinson's conjecture reads

$$
\operatorname{ord}_{s=0} L_{\mathbb{Q}}\left(M_{\eta}, s\right) \stackrel{\sqrt[3.5]{=}}{=} \operatorname{ord}_{s=0} L_{\mathbb{Q}}(E, s)=\sum_{a \neq 1}(-1)^{a} \operatorname{dim} \mathrm{H}^{a}\left(E^{\vee}(-1)\right) .
$$

In fact, $\mathrm{H}^{a}\left(E^{\vee}(-1)\right) \stackrel{(5.10)}{=} \mathrm{H}^{a+2}\left(\eta ! * \eta^{*} \mathrm{~h}^{-b}(X,-n)\right)$. For example, in case $w=$ $2 m-b \leq 1$, this equals $\mathrm{H}^{b+2}\left(X_{\eta}, n\right)_{\mathbb{Z}}$ for $a=0$ and vanishes for $a \neq 0,1$. As was mentioned above, the perfectness of $\pi_{E[-1]}$ conjectured in 4.1]implies $\mathrm{H}^{1}\left(E^{\vee}(-1)\right)=$ 0 . (In case $w \geq 2$, we know this vanishing without invoking 4.1) This settles the pole order part (A] of Beilinson's conjecture.

For the special $L$-values, we revisit the proof of Proposition 5.16 and look at the involved $\mathbb{Q}$-structures. Again using hard Lefschetz, we replace the map $\mathrm{H}^{b+2}\left(X_{\eta}, n\right)_{\mathbb{Z}} \otimes \mathbb{R} \rightarrow \mathrm{H}_{\mathrm{D}}^{b+2}\left(X_{\eta}, n\right)$ occurring in (5.7), (5.8) by $\operatorname{ch}\left(E^{\vee}(-1)\right)$, see (5.11). The involved $\mathbb{Q}$-structures remain unchanged.

We first treat the case $w=1$. By [Fon92, 9.5], FPR94, Conj. III.4.4.3], Beilinson's conjecture is equivalent to saying that the $L$-value of $M_{\eta}$ is given by the reciprocal of the image (in $\mathbb{R}$ ) of the $\mathbb{Q}$-structure on the right hand side:

$$
\begin{aligned}
\mathbb{R} & \cong \operatorname{det}^{-1} \mathrm{H}^{0}\left(E^{\vee}(-1)\right)_{\mathbb{R}} \otimes \operatorname{det}^{-1} \mathrm{H}_{\mathrm{D}}^{1}(E) \otimes \operatorname{det} \mathrm{H}^{1}(E)_{\mathbb{R}} \\
& \stackrel{5.3 .3}{=} \operatorname{det}^{-1} \mathrm{H}^{*}\left(E^{\vee}(-1)\right)_{\mathbb{R}} \otimes \operatorname{det} \mathrm{H}_{\mathrm{D}}^{*}(E) \otimes \operatorname{det}^{-1} \mathrm{H}^{*}(E)_{\mathbb{R}} .
\end{aligned}
$$

Here the isomorphism stems from the exact sequence (5.12) and the $\mathbb{Q}$-structure on $\mathrm{H}_{\mathrm{D}}^{1}(E)$ is the natural one defined in Section 2.1. (This $\mathbb{Q}$-structure is distinct from the one on the isomorphic group $\mathrm{H}_{\mathrm{D}}^{0}\left(E^{\vee}(-1)\right)^{\vee}$, as is apparent from the discussion of 
the functional equation in Theorem [5.5.) Moreover, all groups $\mathrm{H}^{*}\left(E^{\vee}(-1)\right)$ except $\mathrm{H}^{0}$ and $\mathrm{H}^{*}(E)$ except $\mathrm{H}^{1}$ vanish. By construction of Arakelov motivic cohomology the above is isomorphic, including the $\mathbb{Q}$-structure, to

$$
\operatorname{det}^{-1} \mathrm{H}^{*}\left(E^{\vee}(-1)\right)_{\mathbb{R}} \otimes \operatorname{det}^{-1} \widehat{\mathrm{H}}_{\mathbb{R}}^{*}(E) .
$$

The above identification with $\mathbb{R}$ agrees with the dual of the Arakelov intersection pairing for $E$, so that $L^{*}(E, 0)=L^{*}\left(M_{\eta}, 0\right)^{-1}$ is indeed the inverse of $\Pi_{E}$. This accomplishes the case $w=1$.

Again, the case $w \leq 0$ is similar but simpler, since in addition $\mathrm{H}^{1}(E)=0$. Correspondingly, only the determinant of the realization map $\operatorname{ch}\left(E^{\vee}(-1)\right)$ (5.11), as opposed to the one of (5.12), appears in Beilinson's conjecture.

Finally, consider the special value at the central point, i.e., $w=2$. In this case all groups $\mathrm{H}_{\mathrm{D}}^{*}(E)$ are trivial, but the $\mathbb{Q}$-structure on

$$
\operatorname{det}^{-1} \mathrm{H}_{\mathrm{D}}^{*}(E)=\operatorname{det} \mathrm{H}_{\mathrm{D}}^{*}\left(M_{\eta}\right)=\operatorname{det} \underbrace{\mathrm{H}_{\mathrm{B}}^{b+1}\left(X_{\eta}, \mathbb{R}(m)\right)^{(-1)^{m}}}_{=: B} \otimes \operatorname{det}^{-1} \underbrace{\left.\mathrm{H}_{\mathrm{dR}}^{b+1}\left(X_{\eta} \times \mathbb{R}\right) / F^{m}\right)}_{=: d R}
$$

is non-trivial since the period isomorphism $\alpha: B \rightarrow d R$ does not respect the natural $\mathbb{Q}$-structures. By linear algebra, det $\alpha$ agrees (modulo $\mathbb{Q}^{\times}$) with the image (in $\mathbb{R}$ ) of the $\mathbb{Q}$-lattice under the natural isomorphism induced by $\alpha$ : $\operatorname{det} B \otimes \operatorname{det}^{-1} d R \stackrel{\cong}{\rightrightarrows} \mathbb{R}$. Except for $\mathrm{H}^{2}(E)$ and $\mathrm{H}^{0}\left(E^{\vee}(-1)\right)$, all motivic cohomology groups of $E$ and $E^{\vee}(-1)$ vanish (Theorem [1.3). The Arakelov intersection pairing $\pi_{E[-2]}$ agrees with the height pairing under Conjecture 5.6. By (2.17), we have an isomorphism of $\mathbb{R}$ vector spaces respecting the $\mathbb{Q}$-structure

$$
\operatorname{det} \widehat{\mathrm{H}}_{\mathbb{R}}^{*}(E)=\operatorname{det} \mathrm{H}^{*}(E)_{\mathbb{R}} \otimes \operatorname{det}^{-1} \mathrm{H}_{\mathrm{D}}^{*}(E),
$$

so Beilinson's conjecture is indeed equivalent to saying that $L^{*}(E, 0)=L^{*}\left(M_{\eta}, 0\right)^{-1}$ is the reciprocal of the image of the $\mathbb{Q}$-lattice under $\operatorname{det} \widehat{H}_{\mathbb{R}}^{*}(E) \otimes \operatorname{det} \mathrm{H}^{*}\left(E^{\vee}(-1)\right)_{\mathbb{R}} \rightarrow$ $\mathbb{R}$.

\subsection{Relation to the Tate conjecture over $\mathbb{F}_{p}$}

Conjecture 5.19. (Tate conjecture over finite fields [Tat65]) Let $X / \mathbb{F}_{q}$ be smooth and projective. Let $\ell$ be a prime such that $\ell \nmid q$. Any $\operatorname{Gal}\left(\mathbb{F}_{q}\right)$-invariant element of $\mathrm{H}^{2 i}\left(X \times_{\mathbb{F}_{q}} \overline{\mathbb{F}_{q}}, \mathbb{Q}_{\ell}(i)\right)$ is a $\mathbb{Q}_{\ell}$-linear combination of algebraic elements, i.e., elements in the image of the cycle class map $\mathrm{CH}^{i}(X) \rightarrow \mathrm{H}^{2 i}\left(X \times_{\mathbb{F}_{q}} \overline{\mathbb{F}_{q}}, \mathbb{Q}_{\ell}(i)\right)$.

Theorem 5.20. In addition to the general assumptions on mixed motives over $\mathbb{F}_{p}$ (Section 1.2), we assume Conjecture 1.5. Then the Tate conjecture 5.19 is equivalent to Conjecture 5.2 for motives $M=i_{*} N$, where $N$ is any geometric motive over $\mathbb{F}_{p}$, $i: \operatorname{Spec} \mathbb{F}_{p} \rightarrow$ Spec $\mathbb{Z}$. More precisely, the special value prediction of 5.2 in this case is

$$
L^{*}\left(i_{*} N, 0\right) \equiv(\log p)^{-\chi\left(N^{\vee}(-1)\right)}\left(\bmod \mathbb{Q}^{\times}\right),
$$

where $\chi\left(N^{\vee}(-1)\right)$ is the Euler characteristic of motivic cohomology (see (5.1), computed in the category $\left.\mathbf{D M}_{\mathrm{b}, c}\left(\mathbb{F}_{p}\right)\right)$.

Proof: $\Rightarrow$ : to show Conjecture 5.2 and (5.15) for $i_{*} N$, we may replace $N$ by $\mathrm{gr}_{*}^{W} \mathrm{p}^{*} N$, the weight graded pieces of the truncations with respect to the motivic $t$-structure, since both the weight filtration and the $t$-structure are bounded Sch12b, Axiom 4.1.]. The subcategory of $\mathbf{M M}\left(\mathbb{F}_{p}\right)$ consisting of pure objects is, by $[\mathrm{Sch} 12 \mathrm{~b}$, Axiom 4.11], the category of pure motives with respect to numerical equivalence, $\mathbf{M}_{\text {num }}\left(\mathbb{F}_{p}\right)$. Under Conjecture 1.5, this agrees with Chow motives $\mathbf{M}_{\mathrm{rat}}\left(\mathbb{F}_{p}\right)$. Finally, $\chi_{\mathbf{D M}_{\mathrm{B}}(\mathbb{Z})}\left(\left(i_{*} N\right)^{\vee}(-1)\right)=\chi_{\mathbf{D M}_{\mathrm{B}}\left(\mathbb{F}_{p}\right)}\left(N^{\vee}(-1)\right)$, so we have 
to show $\operatorname{ord}_{s=0} L\left(i_{*} N\right)=-\operatorname{dim}^{0}\left(N^{\vee}, 1\right)=-\operatorname{dim}_{0}(N,-1)$ and $L^{*}\left(i_{*} N\right) \equiv$ $(\log p)^{-\operatorname{dim} \mathrm{H}_{0}(N,-1)}\left(\bmod \mathbb{Q}^{\times}\right)$.

Consider first $N=H:=\mathrm{M}_{\mathbb{F}_{p}}(X)\{-n\}$ with $X / \mathbb{F}_{p}$ smooth and projective. Then $L\left(i_{*} H\right)=L\left(\mathrm{M}_{\mathbb{Z}}(X)\{1-n\}\right)$. Let $Z^{n}(X) /$ num be the group of codimension $n$ cycles on $X$ modulo numerical equivalence. Then

$$
\operatorname{dim} \mathrm{H}^{0}(H)=\mathrm{rkCH}^{n}(X) \stackrel{1.5}{=} \mathrm{rk} Z^{n}(X) / \mathrm{num}=-\operatorname{ord}_{s=n} \zeta(X, s),
$$

so the pole order claim holds for $H$ by assumption: the Tate conjecture and the agreement of the $\ell$-adic homological and numerical equivalence relations on $X$ (up to torsion) together are equivalent to the rightmost equality [Tat94, Thm. 2.9].

In general, $N$ is a direct summand of $H$ as above. Let $N \oplus N^{\prime}=H$, which as an object in $\mathbf{M}_{\text {rat }}\left(\mathbb{F}_{p}\right)$ is denoted $h(X)(n)$. By the previous case,

$$
\operatorname{dim} \mathrm{H}^{0} N+\operatorname{dim} \mathrm{H}^{0} N^{\prime}=-\operatorname{ord} L(N)-\operatorname{ord} L\left(N^{\prime}\right) .
$$

Let $-_{\ell}: \mathbf{M}_{\text {rat }}\left(\mathbb{F}_{p}\right) \rightarrow \oplus \underline{\mathbb{Q}_{\ell}}\left[\operatorname{Gal}\left(\mathbb{F}_{p}\right)\right], \pi h(X)(n) \mapsto \oplus_{a} \pi^{*} \mathrm{H}^{a}\left(X, \mathbb{Q}_{\ell}(n)\right)$ be the $\ell$-adic realization functor taking values in graded continuous $\ell$-adic $\operatorname{Gal}\left(\mathbb{F}_{p}\right)$ representations. We write $\mathrm{H}^{0}\left(N_{\ell}\right):=N_{\ell}^{\mathrm{Gal}\left(\mathbb{F}_{p}\right)}$, the Galois cohomology of the $\ell$-adic Galois module $N_{\ell}$. The following way of reasoning is borrowed from loc. cit. We have the following chain of inequalities:

$$
\begin{aligned}
-\operatorname{ord}_{s=0} L(N, s) & \geq \operatorname{dim}_{\mathbb{Q}_{\ell}} \operatorname{ker}\left(\mathrm{Id}-\mathrm{Fr}^{-1}\right) \mid N_{\ell} \\
& \geq \operatorname{dim}_{\mathbb{Q}_{\ell}}\left(N_{\ell}\right)^{\operatorname{Gal}\left(\mathbb{F}_{p}\right)} \\
& =\operatorname{dim}_{\mathbb{Q}_{\ell}} \mathrm{H}^{0}\left(N_{\ell}\right) \\
& \geq \operatorname{dim}_{\mathbb{Q}} \mathrm{H}^{0}(N)
\end{aligned}
$$

The last inequality is by the injectivity of the cycle class map $\mathrm{H}^{0}(N) \rightarrow \mathrm{H}^{0}\left(N_{\ell}\right)$, which follows from the injectivity of $\mathrm{H}^{0}(H) \rightarrow \mathrm{H}^{0}\left(H_{\ell}\right)=\mathrm{H}^{2 n}\left(X, \mathbb{Q}_{\ell}(n)\right)$, i.e., the agreement of homological and rational equivalence, which holds under Conjecture 1.5. Therefore, in (5.16) equality of dimensions must hold for the individual summands, so the pole order part is shown.

The claim (5.15) and the special values formula of 5.2 trivially hold for $N=$ $\mathbf{1}(-1)$ : the residue of $L\left(i_{*} \mathbf{1}(-1), s\right)=\zeta\left(\operatorname{Spec} \mathbb{F}_{p}, s\right)=\left(1-p^{-s}\right)^{-1}$ at $s=0$ is $(\log p)^{-1}$, which is the inverse of the determinant of $\pi_{\mathrm{M}\left(\mathbb{F}_{p}\right)}=\pi_{i_{*} i^{*} 1\{-1\}}$ (Example 2.7). Jannsen's semisimplicity theorem for $\mathbf{M}_{\text {num }}\left(\mathbb{F}_{p}\right)$ yields a decomposition $N=$ $\mathbf{1}(-1)^{r} \oplus R$ with $\operatorname{Hom}_{\mathbf{M}_{\text {num }}\left(\mathbb{F}_{p}\right)}(\mathbf{1}(-1), R)=\operatorname{Hom}_{\mathbf{M}_{\text {num }}\left(\mathbb{F}_{p}\right)}(R, \mathbf{1}(-1))=0$. Hence we can assume $N=R$. By the Lefschetz trace formula, the $L$-function of any pure motive over $\mathbb{F}_{p}$ is a rational function in $p^{-s}$ with rational coefficients that are independent of $\ell$, see e.g. And04, Section 7.1.4]. By the preceding part, the $L$-function of $i_{*} R$ does not have a pole at $s=0$, therefore the leading term of the Laurent series $L\left(i_{*} R, s\right)$ is simply the value at this point, a nonzero rational number (as opposed to an $\ell$-adic or, via $\sigma_{\ell}$, a complex number).

$\Leftarrow$ : we again use the theorem of Tate cited above: the Tate conjecture for $X / \mathbb{F}_{p}$ is implied by $\operatorname{ord}_{s=j} \zeta(X, s)=-\operatorname{rk} Z^{j}(X) /$ num. Under 1.5, that term is $-\operatorname{rk} \mathrm{CH}^{j}(X)=-\operatorname{dim} \mathrm{H}^{2 j}(\mathrm{M}(X)(-j))$. Thus, Conjecture 5.2 for $i_{*} \mathrm{M}(X)(-j)$ implies the Tate conjecture on the $j$-th Chow group of $X$.

\section{References}

[And04] Yves André. Une introduction aux motifs, volume 17 of Panoramas et Synthèses. SMF, Paris, 2004. 
[Ayo07] Joseph Ayoub. Les six opérations de Grothendieck et le formalisme des cycles évanescents dans le monde motivique. I. Astérisque, (314):x+466 pp. (2008), 2007.

[Ayo10] Joseph Ayoub. Note sur les opérations de Grothendieck et la réalisation de Betti. J. Inst. Math. Jussieu, 9(2):225-263, 2010.

[Ayo12] Joseph Ayoub. La réalisation étale et les opérations de Grothendieck. Preprint, 2012, available at http://user.math.uzh.ch/ayoub/, 2012.

[Beǐ84] Alexander A. Beulinson. Higher regulators and values of $L$-functions. In Current problems in mathematics, Vol. 24, Itogi Nauki i Tekhniki, pages 181-238. Akad. Nauk SSSR Vsesoyuz. Inst. Nauchn. i Tekhn. Inform., Moscow, 1984.

[Beĭ86] Alexander A. Beĭlinson. Notes on absolute Hodge cohomology. In Applications of algebraic $K$-theory to algebraic geometry and number theory, Part I, II (Boulder, Colo., 1983), volume 55 of Contemp. Math., pages 35-68. Amer. Math. Soc., Providence, RI, 1986.

[Beř87] Alexander A. Beĭlinson. Height pairing between algebraic cycles. In $K$ theory, arithmetic and geometry (Moscow, 1984-1986), volume 1289 of Lecture Notes in Math., pages 1-25. Springer, Berlin, 1987.

[Bon09] Mikhail V. Bondarko. Differential graded motives: weight complex, weight filtrations and spectral sequences for realizations; Voevodsky versus Hanamura. J. Inst. Math. Jussieu, 8(1):39-97, 2009.

[Bon10] Mikhail V. Bondarko. Weight structures vs. $t$-structures; weight filtrations, spectral sequences, and complexes (for motives and in general). $J$. K-Theory, 6(3):387-504, 2010.

[Bor77] Armand Borel. Cohomologie de $\mathrm{SL}_{n}$ et valeurs de fonctions zeta aux points entiers. Ann. Scuola Norm. Sup. Pisa Cl. Sci. (4), 4(4):613-636, 1977.

[Bur94] José Ignacio Burgos. A $C^{\infty}$ logarithmic Dolbeault complex. Compositio Math., 92(1):61-86, 1994.

[CD09] Denis-Charles Cisinski and Frédéric Déglise. Triangulated categories of mixed motives, 2009. Preprint, available at http://arxiv.org/abs/0912.2110.

[Del71] Pierre Deligne. Théorie de Hodge. II. Inst. Hautes Études Sci. Publ. Math., 40:5-57, 1971.

[Del73] Pierre Deligne. Les constantes des équations fonctionnelles des fonctions $L$. In Modular functions of one variable, II (Proc. Internat. Summer School, Univ. Antwerp, Antwerp, 1972), pages 501-597. Lecture Notes in Math., Vol. 349. Springer, Berlin, 1973.

[Del74] Pierre Deligne. La conjecture de Weil. I. Inst. Hautes Études Sci. Publ. Math., 43:273-307, 1974.

[Del79] Pierre Deligne. Valeurs de fonctions $L$ et périodes d'intégrales. In $A u$ tomorphic forms, representations and L-functions (Proc. Sympos. Pure Math., Oregon State Univ., Corvallis, Ore., 1977), Part 2, Proc. Sympos. Pure Math., XXXIII, pages 313-346. Amer. Math. Soc., Providence, R.I., 1979. With an appendix by N. Koblitz and A. Ogus. 
[DN95] Christopher Deninger and Enric Nart. On Ext ${ }^{2}$ of motives over arithmetic curves. Amer. J. Math., 117(3):601-625, 1995.

[EV88] Hélène Esnault and Eckart Viehweg. Deligne-Beŭlinson cohomology. In Beilinson's conjectures on special values of L-functions, volume 4 of Perspect. Math., pages 43-91. Academic Press, Boston, MA, 1988.

[FM12] Matthias Flach and Baptiste Morin. On the weil-étale topos of regular arithmetic schemes. Documenta Math., 17:313-399, 2012.

[Fon92] Jean-Marc Fontaine. Valeurs spéciales des fonctions $L$ des motifs. Astérisque, 206:Exp. No. 751, 4, 205-249, 1992. Séminaire Bourbaki, Vol. $1991 / 92$.

[FPR94] Jean-Marc Fontaine and Bernadette Perrin-Riou. Autour des conjectures de Bloch et Kato: cohomologie galoisienne et valeurs de fonctions $L$. In Motives (Seattle, WA, 1991), volume 55 of Proc. Sympos. Pure Math., pages 599-706. Amer. Math. Soc., Providence, RI, 1994.

[Gei98] Thomas Geisser. Tate's conjecture, algebraic cycles and rational $K$-theory in characteristic $p$. K-Theory, 13(2):109-122, 1998.

[Gei10] Thomas Geisser. Duality via cycle complexes. Ann. of Math. (2), 172(2):1095-1126, 2010.

[GS90a] Henri Gillet and Christophe Soulé. Arithmetic intersection theory. Inst. Hautes Études Sci. Publ. Math., (72):93-174 (1991), 1990.

[GS90b] Henri Gillet and Christophe Soulé. Characteristic classes for algebraic vector bundles with Hermitian metric. II. Ann. of Math. (2), 131(2):205$238,1990$.

[GS94] Henri Gillet and Christophe Soulé. Arithmetic analogs of the standard conjectures. In Motives (Seattle, WA, 1991), volume 55 of Proc. Sympos. Pure Math., pages 129-140. Amer. Math. Soc., Providence, RI, 1994.

[Héb11] David Hébert. Structure de poids à la Bondarko sur les motifs de Beilinson. Compos. Math., 147(5):1447-1462, 2011.

[HS11] Andreas Holmstrom and Jakob Scholbach. Arakelov motivic cohomology I. To appear in Journal of Algebraic Geometry. Preprint (Aug. 2011) available at http://arxiv.org/abs/1012.2523, 2011.

[Hub00] Annette Huber. Realization of Voevodsky's motives. J. Algebraic Geom., $9(4): 755-799,2000$.

[Ivo07] Florian Ivorra. Réalisation $l$-adique des motifs triangulés géométriques. I. Doc. Math., 12:607-671, 2007.

[Jan90] Uwe Jannsen. Mixed motives and algebraic K-theory, volume 1400 of Lecture Notes in Mathematics. Springer-Verlag, Berlin, 1990. With appendices by S. Bloch and C. Schoen.

[Jan92] Uwe Jannsen. Motives, numerical equivalence, and semi-simplicity. Invent. Math., 107(3):447-452, 1992.

[Kah05] Bruno Kahn. Algebraic $K$-theory, algebraic cycles and arithmetic geometry. In Handbook of K-theory. Vol. 1, 2, pages 351-428. Springer, Berlin, 2005 . 
[Lic84] Stephen Lichtenbaum. Values of zeta-functions at nonnegative integers. In Number theory, Noordwijkerhout 1983 (Noordwijkerhout, 1983), volume 1068 of Lecture Notes in Math., pages 127-138. Springer, Berlin, 1984.

[Mil80] James S. Milne. Étale cohomology, volume 33 of Princeton Mathematical Series. Princeton University Press, Princeton, N.J., 1980.

[Mil06] James S. Milne. Arithmetic duality theorems. BookSurge, LLC, Charleston, SC, second edition, 2006.

[Neu92] Jürgen Neukirch. Algebraic number theory. (Algebraische Zahlentheorie.). Berlin etc.: Springer-Verlag. xiii, 595 S. , 1992.

[Qui72] Daniel Quillen. On the cohomology and $K$-theory of the general linear groups over a finite field. Ann. of Math. (2), 96:552-586, 1972.

[Sch88] Peter Schneider. Introduction to the Beylinson conjectures. In Bellinson's conjectures on special values of L-functions, volume 4 of Perspect. Math., pages 1-35. Academic Press, Boston, MA, 1988.

[Sch91] Anthony J. Scholl. Remarks on special values of $L$-functions. In $L$ functions and arithmetic (Durham, 1989), volume 153 of London Math. Soc. Lecture Note Ser., pages 373-392. Cambridge Univ. Press, Cambridge, 1991.

[Sch94] Anthony J. Scholl. Height pairings and special values of $L$-functions. In Motives (Seattle, WA, 1991), volume 55 of Proc. Sympos. Pure Math., pages 571-598. Amer. Math. Soc., Providence, RI, 1994.

[Sch11] Jakob Scholbach. Mixed Artin-Tate motives over number rings. Journal Pure Appl. Alg., 215:2106-2118, 2011. Preprint (Aug. 2010) available at http://arxiv.org/abs/1003.1267.

[Sch12a] Jakob Scholbach. Arakelov motivic cohomology II. To appear in Journal of Algebraic Geometry. Preprint (May 2012) available at http://arxiv.org/abs/1205.3890, 2012.

[Sch12b] Jakob Scholbach. $f$-cohomology and motives over number rings. Kodai Math. J., 35:1-32, 2012. Preprint (Mar. 2010) available at http://arxiv.org/abs/1003.1219.

[Ser65] Jean-Pierre Serre. Zeta and $L$ functions. In Arithmetical Algebraic Geometry (Proc. Conf. Purdue Univ., 1963), pages 82-92. Harper \& Row, New York, 1965.

[Ser78] Jean-Pierre Serre. Représentations linéaires des groupes finis. Hermann, Paris, revised edition, 1978.

[Sou84] Christophe Soulé. $K$-théorie et zéros aux points entiers de fonctions zêta. In Proceedings of the International Congress of Mathematicians, Vol. 1, 2 (Warsaw, 1983), pages 437-445, Warsaw, 1984. PWN.

[Sou92] Christophe Soulé. Lectures on Arakelov geometry, volume 33 of Cambridge Studies in Advanced Mathematics. Cambridge University Press, Cambridge, 1992. With the collaboration of D. Abramovich, J.-F. Burnol and J. Kramer.

[Tak05] Yuichiro Takeda. Higher arithmetic K-theory. Publ. Res. Inst. Math. Sci., 41(3):599-681, 2005. 
[Tat65] John T. Tate. Algebraic cycles and poles of zeta functions. In Arithmetical Algebraic Geometry (Proc. Conf. Purdue Univ., 1963), pages 93-110. Harper \& Row, New York, 1965.

[Tat94] John Tate. Conjectures on algebraic cycles in l-adic cohomology. In Motives (Seattle, WA, 1991), volume 55 of Proc. Sympos. Pure Math., pages 71-83. Amer. Math. Soc., Providence, RI, 1994.

[Tot14] Burt Totaro. Chow groups, Chow cohomology, and linear varieties. Forum Math. Sigma, 2:e17, 25, 2014.

[Voe00] Vladimir Voevodsky. Triangulated categories of motives over a field. In Cycles, transfers, and motivic homology theories, volume 143 of Ann. of Math. Stud., pages 188-238. Princeton Univ. Press, Princeton, NJ, 2000. 